\title{
Amine-Mediated Transimination and Aromatization-Triggered Domino Reaction in the Synthesis of Polyfunctionalized 4-Aminoquinolines
}

\author{
Runzhe Song, Zhaomeng Han, Qiuqin He and Renhua Fan*
}

\section{Supporting Information}

1. General Information (S1)

2. Representative Procedure and Spectral Data of Products (S2-S9)

3. X-Ray Diffraction Structure of Compound 8a (S9)

4. Copies of ${ }^{1} \mathrm{H},{ }^{13} \mathrm{C}$ NMR Spectra of Products (S10-S77) 


\section{General Information}

All reactions were performed in Schlenk tubes under nitrogen atmosphere. Flash column chromatography was performed using silica gel (60- $\AA$ pore size, $32-63 \mu \mathrm{m}$, standard grade). Analytical thin-layer chromatography was performed using glass plates pre-coated with $0.25 \mathrm{~mm}$ 230-400 mesh silica gel impregnated with a fluorescent indicator (254 nm). Thin layer chromatography plates were visualized by exposure to ultraviolet light. Organic solutions were concentrated on rotary evaporators at $\sim 20$ Torr (house vacuum) at $35-40{ }^{\circ} \mathrm{C}$. Commercial reagents and solvents were used as received. Nuclear magnetic resonance (NMR) spectra are recorded in parts per million from internal tetramethylsilane on the $\delta$ scale.

\section{Representative Procedure and Spectral Data of Products}

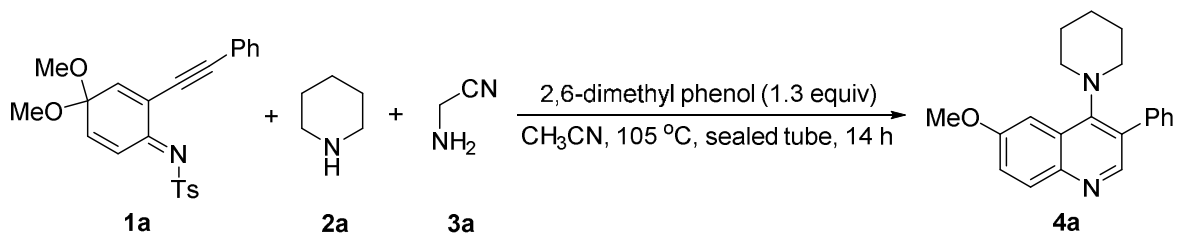

In a sealed tube, a solution of $N$-Ts 2-phenyl-ethynyl quinone imine ketal 1a $(0.1 \mathrm{mmol})$ in acetonitrile $(2.0 \mathrm{~mL})$ was mixed with piperidine $(0.11 \mathrm{mmol}), 2$-aminoacetonitrile $(0.13 \mathrm{mmol})$, and 2,6-dimethylphenol $(0.13 \mathrm{mmol})$ in a nitrogen atmosphere. The resulting mixture in the sealed tube was stirred at $105{ }^{\circ} \mathrm{C}$ for $14 \mathrm{~h}$., then the mixture was concentrated in vacuo. The residue was purified by flash column chromatography on silica gel (eluent: $n$-hexane/ethyl acetate $=12 / 1$ ) to furnish the desired compound $\mathbf{4 a}$

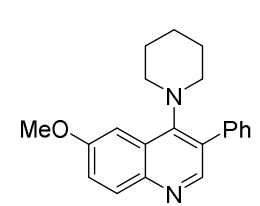
$6 \mathrm{H}){ }^{13} \mathrm{C} \mathrm{NMR}(400 \mathrm{MHz}, \mathrm{CDCl} 3) \delta 157.7,151.0,139.2,130.6,129.9,128.8$, 128.2, 127.3, 127.2, 121.2, 102.6, 55.4, 53.6, 26.8, 24.3; HRMS $\mathrm{m} / \mathrm{z}$ calcd for $\mathrm{C}_{21} \mathrm{H}_{22} \mathrm{~N}_{2} \mathrm{O}\left([\mathrm{M}+\mathrm{H}]^{+}\right): 319.1805$, found 319.1807 .

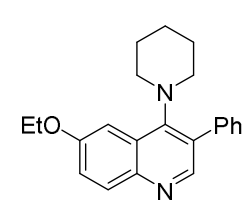

6-ethoxy-3-phenyl-4-(piperidin-1-yl)quinoline $4 \mathbf{b}$ (27 mg, yield 83\%): yellow solid; m.p. $150-152{ }^{\circ} \mathrm{C} ;{ }^{1} \mathrm{H}$ NMR $\left(400 \mathrm{MHz}, \mathrm{CDCl}_{3}\right) \delta 8.47$ (s, $\left.1 \mathrm{H}\right), 7.98$ (d, $J=$ $9.1 \mathrm{~Hz}, 1 \mathrm{H}), 7.48-7.31(\mathrm{~m}, 7 \mathrm{H}), 4.20(\mathrm{q}, J=7.0 \mathrm{~Hz}, 2 \mathrm{H}), 3.03-2.70(\mathrm{~m}, 4 \mathrm{H})$, 1.66-1.64 (m, $6 \mathrm{H}), 1.52$ (t, $J=7.0 \mathrm{~Hz}, 3 \mathrm{H}) ;{ }^{13} \mathrm{C}$ NMR $\left(400 \mathrm{MHz}, \mathrm{CDCl}_{3}\right) \delta$ 157.0, 152.7, 151.4, 144.6, 139.5, 131.1, 130.0, 128.8, 128.2, 127.3, 127.2, 121.4, 103.2, 63.7, 53.6, 26.9, 24.4, 14.8; HRMS m/z calcd for $\mathrm{C}_{22} \mathrm{H}_{24} \mathrm{~N}_{2} \mathrm{O}\left([\mathrm{M}+\mathrm{H}]^{+}\right)$: 333.1961, found 333.1955 . 
3-((3-phenyl-4-(piperidin-1-yl)quinolin-6-yl)oxy)propan-1-ol $4 \mathbf{c}$ (23 $\mathrm{mg}$, yield

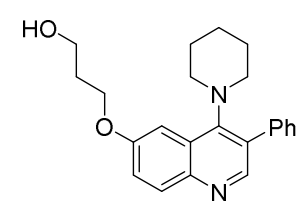
64\%): yellow solid; m.p. $145-147{ }^{\circ} \mathrm{C} ;{ }^{1} \mathrm{H}$ NMR (400 MHz, $\left.\mathrm{CDCl}_{3}\right) \delta 8.47$ (s, $1 \mathrm{H}$ ), 7.97 (d, $J=9.1 \mathrm{~Hz}, 1 \mathrm{H}), 7.48-7.40$ (m, $4 \mathrm{H}), 7.31-7.26$ (m, $3 \mathrm{H}), 4.28$ (t, $J=5.9$ $\mathrm{Hz}, 2 \mathrm{H}), 3.94(\mathrm{t}, J=5.9 \mathrm{~Hz}, 2 \mathrm{H}), 2.95-2.75(\mathrm{~m}, 4 \mathrm{H}), 2.16(\mathrm{~m}, 2 \mathrm{H}), 1.65-1.57$ $(\mathrm{m}, 6 \mathrm{H}) ;{ }^{13} \mathrm{C} \mathrm{NMR}\left(400 \mathrm{MHz}, \mathrm{CDCl}_{3}\right) \delta 156.8,152.8,151.3,144.4,139.3,130.9$, $129.9,128.8,128.2,127.3,127.2,121.3,103.3,65.5,60.0,53.6,32.0,26.8,24.3$; HRMS m/z calcd for $\mathrm{C}_{23} \mathrm{H}_{26} \mathrm{~N}_{2} \mathrm{O}_{2}\left([\mathrm{M}+\mathrm{H}]^{+}\right)$: 363.2067 , found 363.2072.

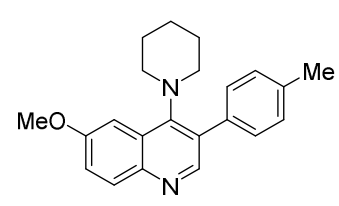

6-methoxy-4-(piperidin-1-yl)-3-( $p$-tolyl)quinoline 4d (21 mg, yield 72\%): yellow ${ }^{\text {Me }}$ solid; m.p. $155-157{ }^{\circ} \mathrm{C} ;{ }^{1} \mathrm{H}$ NMR (400 MHz, $\left.\mathrm{CDCl}_{3}\right) \delta 8.48(\mathrm{~s}, 1 \mathrm{H}), 7.98(\mathrm{~d}, J=$ $9.1 \mathrm{~Hz}, 1 \mathrm{H}), 7.48(\mathrm{~d}, J=2.8 \mathrm{~Hz}, 1 \mathrm{H}), 7.32(\mathrm{dd}, J=9.1 \mathrm{~Hz}, 2.8 \mathrm{~Hz}, 1 \mathrm{H}), 7.26$ (d, $J=7.9 \mathrm{~Hz}, 2 \mathrm{H}), 7.20$ (d, $J=8.0,2 \mathrm{H}), 3.96$ (s, $3 \mathrm{H}), 3.16-2.60$ (m, $4 \mathrm{H}), 2.44$ (s, 3 $\mathrm{H}), 1.80-1.47(\mathrm{~m}, 6 \mathrm{H}) ;{ }^{13} \mathrm{C} \mathrm{NMR}\left(400 \mathrm{MHz}, \mathrm{CDCl}_{3}\right) \delta 157.6,152.6,151.8,144.7$, $137.0,136.5,131.2,129.9,128.9,128.9,127.3,120.9$, 102.5, 55.5, 53.6, 26.9, 24.4, 21.3; HRMS m/z calcd for $\mathrm{C}_{22} \mathrm{H}_{24} \mathrm{~N}_{2} \mathrm{O}\left([\mathrm{M}+\mathrm{H}]^{+}\right)$: 333.1961 , found 333.1954.

6-methoxy-3-(4-pentylphenyl)-4-(piperidin-1-yl)quinoline 4e (30 mg, yield 76\%):

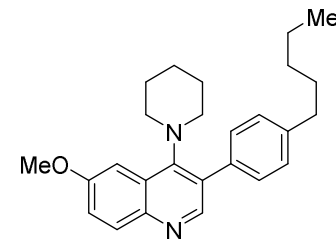

yellow solid; m.p. $101-103{ }^{\circ} \mathrm{C} ;{ }^{1} \mathrm{H}$ NMR (400 MHz, $\left.\mathrm{CDCl}_{3}\right) \delta 8.50(\mathrm{~s}, 1 \mathrm{H}), 7.98$ $(\mathrm{d}, J=9.1 \mathrm{~Hz}, 1 \mathrm{H}), 7.48(\mathrm{~d}, J=2.8 \mathrm{~Hz}, 1 \mathrm{H}), 7.32(\mathrm{dd}, J=9.1 \mathrm{~Hz}, 2.8 \mathrm{~Hz}, 1 \mathrm{H})$, $7.26(\mathrm{~d}, J=8.0 \mathrm{~Hz}, 2 \mathrm{H}), 7.21$ (d, $J=8.0 \mathrm{~Hz}, 2 \mathrm{H}), 3.96$ (s, $3 \mathrm{H}), 2.94-2.78$ (m, 4 $\mathrm{H}), 2.68(\mathrm{t}, J=7.8 \mathrm{~Hz}, 2 \mathrm{H}), 1.69-1.66(\mathrm{~m}, 8 \mathrm{H}), 1.39-1.35(\mathrm{~m}, 4 \mathrm{H}), 0.92$ (t, $J=$ $6.7 \mathrm{~Hz}, 3 \mathrm{H}) ;{ }^{13} \mathrm{C} \mathrm{NMR}\left(400 \mathrm{MHz}, \mathrm{CDCl}_{3}\right) \delta 157.6,152.6,151.8,144.7,142.1$, $136.6,131.2$, 129.8, 129.0, 128.2, 127.3, 120.9, 102.5, 55.5, 53.5, 35.7, 31.6, 31.1, 26.9, 24.4, 22.6, 14.1; HRMS m/z calcd for $\mathrm{C}_{26} \mathrm{H}_{32} \mathrm{~N}_{2} \mathrm{O}\left([\mathrm{M}+\mathrm{H}]^{+}\right): 389.2587$, found 389.2609 .

3-(4-fluorophenyl)-6-methoxy-4-(piperidin-1-yl)quinoline $\mathbf{4 f}$ (26 mg, yield 78\%):

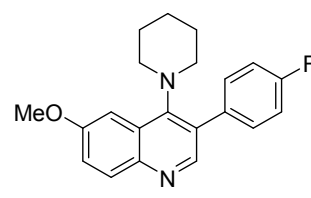

yellow solid; m.p. $149-151{ }^{\circ} \mathrm{C} ;{ }^{1} \mathrm{H}$ NMR $\left(400 \mathrm{MHz}, \mathrm{CDCl}_{3}\right) \delta 8.46(\mathrm{~s}, 1 \mathrm{H}), 8.00$ $(\mathrm{d}, J=9.1 \mathrm{~Hz}, 1 \mathrm{H}), 7.46(\mathrm{~d}, J=2.7 \mathrm{~Hz}, 1 \mathrm{H}), 7.34(\mathrm{dd}, J=9.1,2.8 \mathrm{~Hz}, 1 \mathrm{H})$, 7.30-7.26 (m, $2 \mathrm{H}), 7.16(\mathrm{t}, J=8.6 \mathrm{~Hz}, 2 \mathrm{H}), 3.97(\mathrm{~s}, 3 \mathrm{H}), 2.86(\mathrm{~m}, 4 \mathrm{H})$, $1.69-1.59(\mathrm{~m}, 6 \mathrm{H}) ;{ }^{13} \mathrm{C} \mathrm{NMR}\left(400 \mathrm{MHz}, \mathrm{CDCl}_{3}\right) \delta 162.3(\mathrm{~d}, J(\mathrm{C}, \mathrm{F})=246.7 \mathrm{~Hz})$, $157.8,153.0,151.1,144.5,135.2,131.5(\mathrm{~d}, J(\mathrm{C}, \mathrm{F})=8.0 \mathrm{~Hz}), 130.9,127.8,127.2$, 121.3, $115.3(\mathrm{~d}, J(\mathrm{C}, \mathrm{F})=21.4 \mathrm{~Hz}), 102.6,55.5,53.6,26.8,24.3 ; \mathrm{HRMS} \mathrm{m} / \mathrm{z}$ calcd for $\mathrm{C}_{21} \mathrm{H}_{21} \mathrm{FN}_{2} \mathrm{O}\left([\mathrm{M}+\mathrm{H}]^{+}\right): 337.1711$, found 337.1701 .

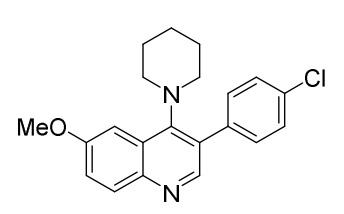

3-(4-chlorophenyl)-6-methoxy-4-(piperidin-1-yl)quinoline 4g (21 mg, yield 60\%): yellow solid; m.p. $148-150{ }^{\circ} \mathrm{C} ;{ }^{1} \mathrm{H}$ NMR (400 MHz, $\left.\mathrm{CDCl}_{3}\right) \delta 8.44(\mathrm{~s}, 1 \mathrm{H}), 7.98$ $(\mathrm{d}, J=9.1 \mathrm{~Hz}, 1 \mathrm{H}), 7.45-7.43$ (m, $3 \mathrm{H}), 7.34$ (d, $J=9.1 \mathrm{~Hz}, 1 \mathrm{H}), 7.25$ (d, $J=8.4$ $\mathrm{Hz}, 2 \mathrm{H}), 3.97$ (s, $3 \mathrm{H}), 3.20-2.50(\mathrm{~m}, 4 \mathrm{H}), 1.68-1.61(\mathrm{~m}, 6 \mathrm{H}) ;{ }^{13} \mathrm{C}$ NMR (400 $\left.\mathrm{MHz}, \mathrm{CDCl}_{3}\right) \delta 157.7,152.7,151.1,144.9,137.9,133.5,131.2,128.5,127.6$, 
127.1, 121.3, 102.5, 55.5, 53.7, 26.8, 24.3; HRMS m/z calcd for $\mathrm{C}_{21} \mathrm{H}_{21} \mathrm{ClN}_{2} \mathrm{O}$ $\left([\mathrm{M}+\mathrm{H}]^{+}\right): 353.1415$, found 353.1410 .

3-(4-bromophenyl)-6-methoxy-4-(piperidin-1-yl)quinoline 4h (22 mg, yield 57\%): (1) Br yellow solid; m.p. $148-150{ }^{\circ} \mathrm{C} ;{ }^{1} \mathrm{H}$ NMR (400 MHz, $\left.\mathrm{CDCl}_{3}\right) \delta 8.43$ (s, $1 \mathrm{H}$ ), 7.98 $(\mathrm{d}, J=9.1 \mathrm{~Hz}, 1 \mathrm{H}), 7.58(\mathrm{~d}, J=8.3 \mathrm{~Hz}, 2 \mathrm{H}), 7.45(\mathrm{~d}, J=2.8 \mathrm{~Hz}, 1 \mathrm{H}), 7.33(\mathrm{dd}, J$ $=9.1 \mathrm{~Hz}, 2.8 \mathrm{~Hz}, 1 \mathrm{H}), 7.18(\mathrm{~d}, J=8.3 \mathrm{~Hz}, 2 \mathrm{H}), 3.96(\mathrm{~s}, 3 \mathrm{H}), 3.15-2.59(\mathrm{~m}, 4 \mathrm{H})$, 1.68-1.60 (m, $6 \mathrm{H}) ;{ }^{13} \mathrm{C} \mathrm{NMR}\left(400 \mathrm{MHz}, \mathrm{CDCl}_{3}\right) \delta 157.6,152.6,150.8,144.7$, $138.3,131.5,131.3,131.0,127.4,127.0,121.5,121.2$, 102.4, 55.4, 53.6, 26.7, 24.2; HRMS m/z calcd for $\mathrm{C}_{21} \mathrm{H}_{21} \mathrm{BrN}_{2} \mathrm{O}\left([\mathrm{M}+\mathrm{H}]^{+}\right)$: 397.0910, found 397.0916.

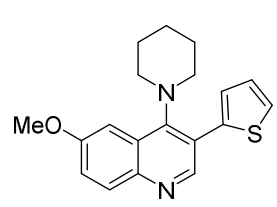

6-methoxy-4-(piperidin-1-yl)-3-(thiophen-2-yl)quinoline 4i (18 mg, yield 54\%): yellow oil; ${ }^{1} \mathrm{H}$ NMR (400 MHz, $\left.\mathrm{CDCl}_{3}\right) \delta 8.61(\mathrm{~s}, 1 \mathrm{H}), 8.02(\mathrm{~d}, J=9.1 \mathrm{~Hz}, 1 \mathrm{H})$, $7.47(\mathrm{~d}, J=2.8 \mathrm{~Hz}, 1 \mathrm{H}), 7.42(\mathrm{dd}, J=5.1 \mathrm{~Hz}, 1.0 \mathrm{~Hz}, 1 \mathrm{H}), 7.33(\mathrm{dd}, J=9.1 \mathrm{~Hz}$, $2.8 \mathrm{~Hz}, 1 \mathrm{H}), 7.13(\mathrm{dd}, J=5.1 \mathrm{~Hz}, 3.5 \mathrm{~Hz}, 1 \mathrm{H}), 7.07$ (d, $J=3.4 \mathrm{~Hz}, 1 \mathrm{H}), 3.97$ (s, $3 \mathrm{H}), 3.20-2.83(\mathrm{~m}, 4 \mathrm{H}), 1.74-1.62(\mathrm{~m}, 6 \mathrm{H}) ;{ }^{13} \mathrm{C} \mathrm{NMR}\left(400 \mathrm{MHz}, \mathrm{CDCl}_{3}\right) \delta$ $157.8,153.8,151.2,144.7,139.8,131.0,127.8,127.3,127.1,126.4,121.9,121.3$, 102.7, 55.5, 52.8, 26.7, 24.3; HRMS m/z calcd for $\mathrm{C}_{19} \mathrm{H}_{20} \mathrm{~N}_{2} \mathrm{OS}\left([\mathrm{M}+\mathrm{H}]^{+}\right)$: 325.1369 , found 325.1369 .

6-methoxy-4-(2-methylpiperidin-1-yl)-3-phenylquinoline 4j (27 mg, yield 82\%): yellow solid; m.p. $137-139{ }^{\circ} \mathrm{C} ;{ }^{1} \mathrm{H}$ NMR $\left(400 \mathrm{MHz}, \mathrm{CDCl}_{3}\right) \delta 8.53(\mathrm{~s}, 1 \mathrm{H}), 8.01$ $(\mathrm{d}, J=9.1 \mathrm{~Hz}, 1 \mathrm{H}), 7.80-7.60(\mathrm{~m}, 1 \mathrm{H}), 7.44-7.33(\mathrm{~m}, 6 \mathrm{H}), 3.95(\mathrm{~s}, 3 \mathrm{H})$, 3.37-3.21 (m, $1 \mathrm{H}), 2.95-2.93(\mathrm{~m}, 1 \mathrm{H}), 2.55-2.35(\mathrm{~m}, 1 \mathrm{H}), 1.76-1.64(\mathrm{~m}, 4 \mathrm{H})$, 1.29-1.26 (m, $2 \mathrm{H}), 0.71(\mathrm{~m}, 3 \mathrm{H}) ;{ }^{13} \mathrm{C} \mathrm{NMR}\left(400 \mathrm{MHz}, \mathrm{CDCl}_{3}\right) \delta$ 157.9, 151.0, $145.1,139.0,132.7,130.9,130.0,129.7,128.0,127.5,121.1,102.8,55.3,54.8$, 52.7, 35.3, 27.0, 24.5, 20.7; HRMS m/z calcd for $\mathrm{C}_{22} \mathrm{H}_{24} \mathrm{~N}_{2} \mathrm{O}\left([\mathrm{M}+\mathrm{H}]^{+}\right)$: 333.1961, found 333.1957 .

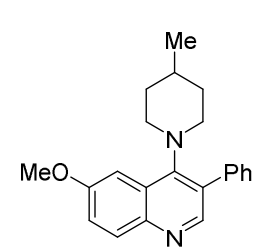

6-methoxy-4-(4-methylpiperidin-1-yl)-3-phenylquinoline 4k (28 mg, yield 86\%): yellow solid; m.p. 120-122 ${ }^{\circ} \mathrm{C} ;{ }^{1} \mathrm{H}$ NMR (400 MHz, $\left.\mathrm{CDCl}_{3}\right) \delta 8.49$ (s, $\left.1 \mathrm{H}\right), 7.99$ $(\mathrm{d}, J=9.1 \mathrm{~Hz}, 1 \mathrm{H}), 7.47-7.30(\mathrm{~m}, 7 \mathrm{H}), 3.96(\mathrm{~s}, 3 \mathrm{H}), 3.17-3.14(\mathrm{~m}, 2 \mathrm{H})$, 2.63-2.57 (m, $2 \mathrm{H}), 1.64-1.62(\mathrm{~m}, 2 \mathrm{H}), 1.38-1.34(\mathrm{~m}, 3 \mathrm{H}), 0.97$ (d, $J=6.1 \mathrm{~Hz}, 3$ $\mathrm{H}) ;{ }^{13} \mathrm{C} \mathrm{NMR}\left(400 \mathrm{MHz}, \mathrm{CDCl}_{3}\right) \delta 157.6,152.5,151.4,144.6,139.4,131.1$, $129.9,129.0,128.2,127.3,127.1,120.9,102.6,55.4,52.8,35.2,30.8,22.2$; HRMS m/z calcd for $\mathrm{C}_{22} \mathrm{H}_{24} \mathrm{~N}_{2} \mathrm{O}\left([\mathrm{M}+\mathrm{H}]^{+}\right): 333.1961$, found 333.1949 .

\footnotetext{
4-(2-ethylpiperidin-1-yl)-6-methoxy-3-phenylquinoline 41 (27 mg, yield 86\%): MeO $(\mathrm{d}, J=9.1 \mathrm{~Hz}, 1 \mathrm{H}), 7.67$ (s, $1 \mathrm{H}), 7.47-7.33$ (m, $6 \mathrm{H}), 3.95$ (s, $3 \mathrm{H}), 3.40-3.20$ (m,
} 
$1 \mathrm{H}), 3.00-2.94(\mathrm{~m}, 1 \mathrm{H}), 2.28(\mathrm{~m}, 1 \mathrm{H}), 1.78-1.65$ (m, $4 \mathrm{H}), 1.26-1.03(\mathrm{~m}, 4 \mathrm{H})$, 0.49 (m, $3 \mathrm{H}) ;{ }^{13} \mathrm{C}$ NMR $\left(400 \mathrm{MHz}, \mathrm{CDCl}_{3}\right) \delta 157.8,151.1,151.0,145.2,139.0$, 132.6, 131.0, 130.1, 129.2, 128.0, 127.5, 121.1, 102.8, 58.2, 55.4, 54.8, 30.7, 27.0, 26.0, 24.1, 9.5; HRMS m/z calcd for $\mathrm{C}_{23} \mathrm{H}_{26} \mathrm{~N}_{2} \mathrm{O}\left([\mathrm{M}+\mathrm{H}]^{+}\right): 347.2118$, found 347.2122 .

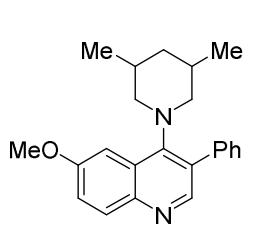

4-(3,5-dimethylpiperidin-1-yl)-6-methoxy-3-phenylquinoline $4 \mathbf{m}$ (31 mg, yield 90\%): yellow solid; m.p. $132-134{ }^{\circ} \mathrm{C} ;{ }^{1} \mathrm{H}$ NMR (400 MHz, $\left.\mathrm{CDCl}_{3}\right) \delta 8.50(\mathrm{~s}, 1 \mathrm{H})$, $7.98(\mathrm{~d}, J=9.1 \mathrm{~Hz}, 1 \mathrm{H}), 7.47-7.30(\mathrm{~m}, 7 \mathrm{H}), 3.93(\mathrm{~s}, 3 \mathrm{H}), 3.12(\mathrm{~d}, J=11.5 \mathrm{~Hz}, 2$ H), 2.13-2.08 (m, $2 \mathrm{H}), 1.87-1.80(\mathrm{~m}, 3 \mathrm{H}), 0.77$ (d, $J=6.4 \mathrm{~Hz}, 6 \mathrm{H}), 0.67-0.59$ $(\mathrm{m}, 1 \mathrm{H}) ;{ }^{13} \mathrm{C} \mathrm{NMR}\left(400 \mathrm{MHz}, \mathrm{CDCl}_{3}\right) \delta 157.6,152.1,151.5,144.9,139.3,131.2$, $129.9,129.0,128.2,127.3,127.2,121.0,102.4,60.1,55.4,42.3,32.3,19.0$; HRMS m/z calcd for $\mathrm{C}_{23} \mathrm{H}_{26} \mathrm{~N}_{2} \mathrm{O}\left([\mathrm{M}+\mathrm{H}]^{+}\right): 347.2118$, found 347.2129.

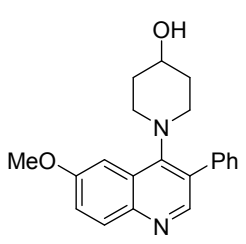

1-(6-methoxy-3-phenylquinolin-4-yl)piperidin-4-ol 4n (28 mg, yield 85\%): yellow solid; m.p. 206-208 ${ }^{\circ} \mathrm{C} ;{ }^{1} \mathrm{H}$ NMR (400 MHz, $\left.\mathrm{CDCl}_{3}\right) \delta 8.47$ (s, $\left.1 \mathrm{H}\right), 7.99$ (d, $J=$ 9.1 Hz, $1 \mathrm{H}), 7.47-7.29$ (m, $7 \mathrm{H}), 3.93$ (m, $3 \mathrm{H}), 3.79-3.75$ (m, $1 \mathrm{H}), 3.16(\mathrm{~m}, 2 \mathrm{H})$, 2.70-2.66 (m, $2 \mathrm{H}), 1.97-1.94$ (m, $2 \mathrm{H}), 1.73-1.71$ (m, $2 \mathrm{H}) ;{ }^{13} \mathrm{C}$ NMR (400 MHz, $\left.\mathrm{CDCl}_{3}\right) \delta 157.8,152.0,151.2,144.6,139.1,131.1,129.8,129.3,128.3,127.5$, 127.0, 121.2, 102.2, 55.4, 50.2, 35.5, 29.6; HRMS m/z calcd for $\mathrm{C}_{21} \mathrm{H}_{22} \mathrm{~N}_{2} \mathrm{O}_{2}$ $\left([\mathrm{M}+\mathrm{H}]^{+}\right): 335.1754$, found 335.1764 .

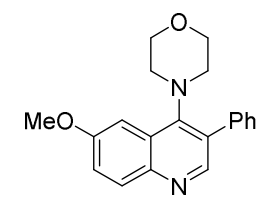

4-(6-methoxy-3-phenylquinolin-4-yl)morpholine 4o (21 mg, yield 70\%): yellow solid; m.p. 164-166 ${ }^{\circ} \mathrm{C} ;{ }^{1} \mathrm{H}$ NMR $\left(400 \mathrm{MHz}, \mathrm{CDCl}_{3}\right) \delta 8.52(\mathrm{~s}, 1 \mathrm{H}), 8.02(\mathrm{~d}, J=$ $9.1 \mathrm{~Hz}, 1 \mathrm{H}), 7.50-7.33(\mathrm{~m}, 7 \mathrm{H}), 3.96(\mathrm{~s}, 3 \mathrm{H}), 3.80$ (t, $J=4.4 \mathrm{~Hz}, 4 \mathrm{H}), 2.94$ (t, $J$ $=4.1 \mathrm{~Hz}, 4 \mathrm{H}) ;{ }^{13} \mathrm{C} \mathrm{NMR}\left(400 \mathrm{MHz}, \mathrm{CDCl}_{3}\right) \delta 157.9,151.4,150.9,144.9,138.7$, 131.4, 129.9, 129.5, 128.5, 127.7, 121.1, 102.4, 67.6, 55.5, 52.4; HRMS m/z calcd for $\mathrm{C}_{20} \mathrm{H}_{20} \mathrm{~N}_{2} \mathrm{O}_{2}\left([\mathrm{M}+\mathrm{H}]^{+}\right)$: 321.1598 , found 321.1595 .

6-methoxy- $N, N$-dimethyl-3-phenylquinolin-4-amine 4 p (14 mg, yield 52\%): $\mathrm{Me}_{{ }_{N}{ }^{-M e}}$ yellow solid; m.p. $47-50{ }^{\circ} \mathrm{C} ;{ }^{1} \mathrm{H}$ NMR $\left(400 \mathrm{MHz}, \mathrm{CDCl}_{3}\right) \delta 8.51(\mathrm{~s}, 1 \mathrm{H}), 8.01(\mathrm{~d}$, $J=9.1 \mathrm{~Hz}, 1 \mathrm{H}), 7.49-7.32(\mathrm{~m}, 7 \mathrm{H}), 3.97(\mathrm{~s}, 3 \mathrm{H}), 2.77(\mathrm{~s}, 6 \mathrm{H}) ;{ }^{13} \mathrm{C} \mathrm{NMR}(400$ $\left.\mathrm{MHz}, \mathrm{CDCl}_{3}\right) \delta 157.5,153.1,151.1,144.6,139.1,130.9,129.6,128.8,128.7$, 128.4, 127.3, 121.0, 103.2, 55.5, 44.3; HRMS m/z calcd for $\mathrm{C}_{18} \mathrm{H}_{18} \mathrm{~N}_{2} \mathrm{O}\left([\mathrm{M}+\mathrm{H}]^{+}\right)$: 279.1492 , found 279.1488 .

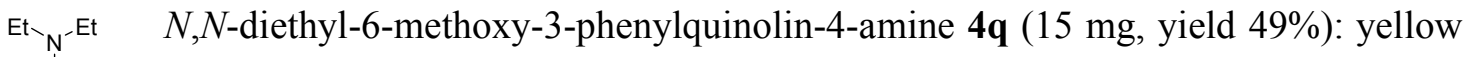
oil; ${ }^{1} \mathrm{H}$ NMR (400 MHz, $\left.\mathrm{CDCl}_{3}\right) \delta 8.50(\mathrm{~s}, 1 \mathrm{H}), 8.00(\mathrm{~d}, J=9.1 \mathrm{~Hz}, 1 \mathrm{H})$, 7.51-7.33 (m, $7 \mathrm{H}), 3.96$ (s, $3 \mathrm{H}), 3.01$ (q, $J=7.1 \mathrm{~Hz}, 4 \mathrm{H}), 1.04$ (t, $J=7.1 \mathrm{~Hz}, 6$ $\mathrm{H}) ;{ }^{13} \mathrm{C} \mathrm{NMR}\left(400 \mathrm{MHz}, \mathrm{CDCl}_{3}\right) \delta 157.6,151.5,151.0,139.1,130.9,129.8$, 
129.6, 128.9, 128.3, 127.4, 121.1, 103.4, 55.5, 47.1, 14.2; HRMS m/z calcd for $\mathrm{C}_{20} \mathrm{H}_{22} \mathrm{~N}_{2} \mathrm{O}\left([\mathrm{M}+\mathrm{H}]^{+}\right): 307.1805$, found 307.1797 .

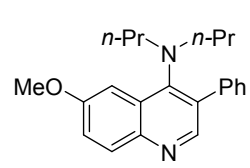

6-methoxy-3-phenyl- $N, N$-dipropylquinolin-4-amine $4 \mathbf{r} \quad(15 \mathrm{mg}$, yield 48\%): yellow oil; ${ }^{1} \mathrm{H}$ NMR $\left(400 \mathrm{MHz}, \mathrm{CDCl}_{3}\right) \delta 8.48(\mathrm{~s}, 1 \mathrm{H}), 8.00(\mathrm{dd}, J=9.0 \mathrm{~Hz}, 3.9$ $\mathrm{Hz}, 1 \mathrm{H}), 7.55-7.32$ (m, 7 H), 3.95 (s, $3 \mathrm{H}), 2.89$ (t, $J=7.6$ Hz, 4 H), 1.49-1.46 (m, $4 \mathrm{H}), 0.78(\mathrm{t}, J=7.4 \mathrm{~Hz}, 6 \mathrm{H}),{ }^{13} \mathrm{C} \mathrm{NMR}\left(400 \mathrm{MHz}, \mathrm{CDCl}_{3}\right) \delta 157.5,151.7,151.1$, $145.1,139.3,131.0,130.6,129.6,128.6,128.3,127.4,121.1,103.4,55.5,55.5$, 22.1, 11.6; HRMS m/z calcd for $\mathrm{C}_{22} \mathrm{H}_{26} \mathrm{~N}_{2} \mathrm{O}\left([\mathrm{M}+\mathrm{H}]^{+}\right)$: 335.2118 , found 335.2121.

$N$-cyclohexyl-6-methoxy- $N$-methyl-3-phenylquinolin-4-amine $4 s$ (18 mg, yield

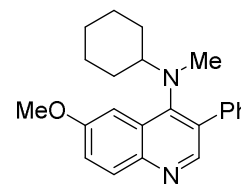
52\%): yellow solid; m.p. $113-115{ }^{\circ} \mathrm{C} ;{ }^{1} \mathrm{H}$ NMR (400 MHz, $\left.\mathrm{CDCl}_{3}\right) \delta 8.49$ (s, $1 \mathrm{H}$ ), $8.01(\mathrm{~d}, J=9.0 \mathrm{~Hz}, 1 \mathrm{H}), 7.47-7.32(\mathrm{~m}, 7 \mathrm{H}), 3.94(\mathrm{~s}, 3 \mathrm{H}), 3.65-3.58(\mathrm{~m}, 1 \mathrm{H})$, $2.80(\mathrm{~s}, 3 \mathrm{H}), 1.66-1.60(\mathrm{~m}, 5 \mathrm{H}), 1.52-1.42(\mathrm{~m}, 1 \mathrm{H}), 1.05-1.04(\mathrm{~m}, 4 \mathrm{H}) ;{ }^{13} \mathrm{C}$ $\operatorname{NMR}\left(400 \mathrm{MHz}, \mathrm{CDCl}_{3}\right) \delta 157.5,152.2,150.6,144.9,139.2,131.2,130.7,129.7$, 129.0, 128.2, 127.3, 121.1, 103.4, 60.5 55.4, 38.2, 31.0, 25.7, 25.1; HRMS m/z calcd for $\mathrm{C}_{23} \mathrm{H}_{26} \mathrm{~N}_{2} \mathrm{O}\left([\mathrm{M}+\mathrm{H}]^{+}\right): 347.2118$, found 347.2115 .

6-methoxy-2-methyl-3-phenyl-4-(piperidin-1-yl)quinoline 4t (16 mg, yield 58\%): yellow solid; m.p. $128-131{ }^{\circ} \mathrm{C} ;{ }^{1} \mathrm{H}$ NMR $\left(400 \mathrm{MHz}, \mathrm{CDCl}_{3}\right) \delta 7.94(\mathrm{~d}, J=9.1 \mathrm{~Hz}$, MeO $1 \mathrm{H}), 7.48-7.40(\mathrm{~m}, 4 \mathrm{H}), 7.32-7.21(\mathrm{~m}, 3 \mathrm{H}), 3.95(\mathrm{~s}, 3 \mathrm{H}), 3.04-2.81(\mathrm{~m}, 1 \mathrm{H})$, 2.50-2.02 (m, $3 \mathrm{H}), 2.35(\mathrm{~s}, 3 \mathrm{H}), 1.80-1.39(\mathrm{~m}, 6 \mathrm{H}) ;{ }^{13} \mathrm{C} \mathrm{NMR}(400 \mathrm{MHz}$, $\left.\mathrm{CDCl}_{3}\right) \delta 157.1,156.7,153.3,144.1,139.3,130.5,130.2,130.0,128.2,127.2$, $126.3,120.8,102.7,55.4,53.3,26.9,24.8,24.2 ; \mathrm{HRMS} \mathrm{m} / \mathrm{z}$ calcd for $\mathrm{C}_{22} \mathrm{H}_{24} \mathrm{~N}_{2} \mathrm{O}$ $\left([\mathrm{M}+\mathrm{H}]^{+}\right): 333.1961$, found 333.1958.

4-(6-methoxy-2-methyl-3-phenylquinolin-4-yl)morpholine 4u (15 mg, yield 49\%):

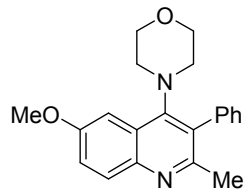
yellow solid; m.p. $145-147{ }^{\circ} \mathrm{C} ;{ }^{1} \mathrm{H}$ NMR $\left(400 \mathrm{MHz}, \mathrm{CDCl}_{3}\right) \delta 7.99(\mathrm{~d}, J=8.7 \mathrm{~Hz}$, $1 \mathrm{H}), 7.50-7.43$ (m, $4 \mathrm{H}), 7.35$ (dd, $J=9.1 \mathrm{~Hz}, 2.7 \mathrm{~Hz}, 1 \mathrm{H}), 7.26-7.22$ (m, $2 \mathrm{H})$, $3.95(\mathrm{~s}, 3 \mathrm{H}), 3.72-3.66(\mathrm{~m}, 4 \mathrm{H}), 2.87-2.77(\mathrm{~m}, 4 \mathrm{H}), 2.37(\mathrm{~s}, 3 \mathrm{H}) ;{ }^{13} \mathrm{C} \mathrm{NMR}$ $\left(400 \mathrm{MHz}, \mathrm{CDCl}_{3}\right) \delta 157.4,156.7,138.5,131.1,130.9,130.5,130.2,128.8,128.5$, 127.7, 125.9, 121.1, 102.5, 67.6, 65.5, 55.4, 52.2; HRMS m/z calcd for $\mathrm{C}_{21} \mathrm{H}_{22} \mathrm{~N}_{2} \mathrm{O}_{2}\left([\mathrm{M}+\mathrm{H}]^{+}\right): 335.1754$, found 335.1760 .

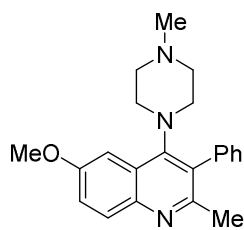

6-methoxy-2-methyl-4-(4-methylpiperazin-1-yl)-3-phenylquinoline $4 \mathbf{v}$ (14 mg, yield 41\%): yellow solid; m.p. $138-141{ }^{\circ} \mathrm{C} ;{ }^{1} \mathrm{H}$ NMR (400 MHz, $\left.\mathrm{CDCl}_{3}\right) \delta 7.93$ (d, $J=9.1 \mathrm{~Hz}, 1 \mathrm{H}), 7.47-7.20$ (m, $7 \mathrm{H}), 3.94$ (s, $3 \mathrm{H}), 2.99-2.90$ (m, $4 \mathrm{H}), 2.69-2.48$ $(\mathrm{m}, 4 \mathrm{H}), 2.34(\mathrm{~s}, 3 \mathrm{H}), 2.28(\mathrm{~s}, 3 \mathrm{H}) ;{ }^{13} \mathrm{C} \mathrm{NMR}\left(400 \mathrm{MHz}, \mathrm{CDCl}_{3}\right) \delta 157.2,156.8$, $152.0,144.2,138.8,130.4,130.2,129.5,128.5,127.4,127.2,120.8,102.7,55.9$, 
55.4, 51.6, 46.3, 24.8; HRMS m/z calcd for $\mathrm{C}_{22} \mathrm{H}_{25} \mathrm{~N}_{3} \mathrm{O}\left([\mathrm{M}+\mathrm{H}]^{+}\right): 348.2070$, found 348.2070 .

6-methoxy-2,3-diphenyl-4-(piperidin-1-yl)quinoline $4 \mathbf{w}$ (17 mg, yield 44\%): yellow solid; m.p. $160-162{ }^{\circ} \mathrm{C} ;{ }^{1} \mathrm{H}$ NMR $\left(400 \mathrm{MHz}, \mathrm{CDCl}_{3}\right) \delta 8.06(\mathrm{~d}, J=9.1 \mathrm{~Hz}$, $1 \mathrm{H}), 7.54(\mathrm{~d}, J=2.7 \mathrm{~Hz}, 1 \mathrm{H}), 7.37-7.34(\mathrm{~m}, 1 \mathrm{H}), 7.25-7.03(\mathrm{~m}, 10 \mathrm{H}), 3.99$ (s, 3 $\mathrm{H}), 3.20-3.12(\mathrm{~m}, 2 \mathrm{H}), 2.50-2.26(\mathrm{~m}, 2 \mathrm{H}), 1.72-1.60(\mathrm{~m}, 6 \mathrm{H}) ;{ }^{13} \mathrm{C}$ NMR $(400$ $\left.\mathrm{MHz}_{2} \mathrm{CDCl}_{3}\right) \delta 158.6,157.6,153.9,144.4,141.5,138.7,131.5,131.4,129.5$, $129.3,127.6,127.3,126.9,126.7,121.2,102.4,55.5,53.2,26.9,24.2 ; \mathrm{HRMS} \mathrm{m} / \mathrm{z}$ calcd for $\mathrm{C}_{27} \mathrm{H}_{26} \mathrm{~N}_{2} \mathrm{O}\left([\mathrm{M}+\mathrm{H}]^{+}\right)$: 395.2118 , found 395.2123 .

3-((2,3-diphenyl-4-(piperidin-1-yl)quinolin-6-yl)oxy)propan-1-ol 4x (16 mg, yield

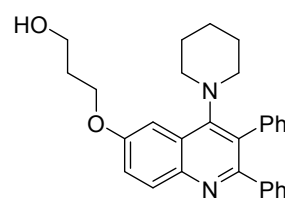
41\%): yellow solid; m.p. 190-192 ${ }^{\circ} \mathrm{C} ;{ }^{1} \mathrm{H}$ NMR (400 MHz, $\left.\mathrm{CDCl}_{3}\right) \delta 8.06$ (d, $J=$ $9.1 \mathrm{~Hz}, 1 \mathrm{H}), 7.54(\mathrm{~d}, J=2.8 \mathrm{~Hz}, 1 \mathrm{H}), 7.34(\mathrm{dd}, J=9.1 \mathrm{~Hz}, 2.8 \mathrm{~Hz}, 1 \mathrm{H})$, 7.26-7.04 (m, $10 \mathrm{H}), 4.30$ (t, $J=5.8 \mathrm{~Hz}, 2 \mathrm{H}), 3.94$ (t, $J=5.9 \mathrm{~Hz}, 2 \mathrm{H}), 3.39-2.85$ (m, $2 \mathrm{H}), 2.43-2.39$ (m, $2 \mathrm{H}), 2.19-2.13(\mathrm{~m}, 2 \mathrm{H}), 1.74-1.48(\mathrm{~m}, 6 \mathrm{H}) ;{ }^{13} \mathrm{C}$ NMR $\left(400 \mathrm{MHz}, \mathrm{CDCl}_{3}\right) \delta 158.6,156.8,154.0,144.3,141.3,138.6,131.5,131.3,129.6$, $129.5,127.6,127.3,127.0,126.8,126.4,121.5,103.2$, 65.7, 60.2, 53.2, 32.0, 26.9, 24.2; HRMS m/z calcd for $\mathrm{C}_{29} \mathrm{H}_{30} \mathrm{~N}_{2} \mathrm{O}_{2}\left([\mathrm{M}+\mathrm{H}]^{+}\right)$: 439.2380, found 439.2378.

6-methyl-3-phenyl-4-(piperidin-1-yl)quinoline 8a (23 mg, yield 74\%): yellow solid; m.p. 163-165 ${ }^{\circ} \mathrm{C} ;{ }^{1} \mathrm{H}$ NMR (400 MHz, $\left.\mathrm{CDCl}_{3}\right) \delta 8.55$ (s, $\left.1 \mathrm{H}\right), 7.97$ (d, $J=$ $8.5 \mathrm{~Hz}, 1 \mathrm{H}), 7.95(\mathrm{~s}, 1 \mathrm{H}), 7.51-7.31(\mathrm{~m}, 6 \mathrm{H}), 3.05-2.75(\mathrm{~m}, 4 \mathrm{H}), 2.58(\mathrm{~s} 3 \mathrm{H})$, $1.67-1.57(\mathrm{~m}, 6 \mathrm{H}) ;{ }^{13} \mathrm{C}$ NMR (400 MHz, $\left.\mathrm{CDCl}_{3}\right) \delta 153.3,153.0,147.4,139.5$, $135.5,130.9,130.0,129.5,128.4,128.2,127.2,126.2$, 123.2, 53.9, 26.6, 24.3, 22.1; HRMS m/z calcd for $\mathrm{C}_{21} \mathrm{H}_{22} \mathrm{~N}_{2}\left([\mathrm{M}+\mathrm{H}]^{+}\right)$: 303.1856 , found 303.1855 .

6-butyl-3-phenyl-4-(piperidin-1-yl)quinoline 8b (15 mg, yield 44\%): yellow oil; ${ }^{1} \mathrm{H}$ NMR (400 MHz, $\left.\mathrm{CDCl}_{3}\right) \delta 8.55(\mathrm{~s}, 1 \mathrm{H}), 7.99(\mathrm{~d}, J=8.5 \mathrm{~Hz}, 1 \mathrm{H}), 7.95(\mathrm{~d}, J=$ $1.3 \mathrm{~Hz}, 1 \mathrm{H}), 7.53-7.30(\mathrm{~m}, 6 \mathrm{H}), 2.94-2.82(\mathrm{~m}, 4 \mathrm{H}), 2.84(\mathrm{t}, J=7.66 \mathrm{~Hz}, 2 \mathrm{H})$, 1.76-1.59 (m, $8 \mathrm{H}), 1.46-1.38(\mathrm{~m}, 2 \mathrm{H}), 0.98(\mathrm{t}, J=7.3 \mathrm{~Hz}, 3 \mathrm{H}) ;{ }^{13} \mathrm{C}$ NMR $(400$ $\left.\mathrm{MHz}, \mathrm{CDCl}_{3}\right) \delta 153.5,152.8,147.2,145.6,140.4,139.5,130.4,130.0,129.2$, $128.2,127.2,126.0,122.6,53.9,35.9,33.3,26.6,24.3,22.2,13.9 ; \mathrm{HRMS} \mathrm{m} / \mathrm{z}$ calcd for $\mathrm{C}_{24} \mathrm{H}_{28} \mathrm{~N}_{2}\left([\mathrm{M}+\mathrm{H}]^{+}\right): 345.2325$, found 345.2331.

6-methyl-4-(piperidin-1-yl)-3-( $p$-tolyl)quinoline 8c (17 mg, yield 54\%): yellow solid; m.p. $128-130{ }^{\circ} \mathrm{C} ;{ }^{1} \mathrm{H}$ NMR (400 MHz, $\left.\mathrm{CDCl}_{3}\right) \delta 8.53(\mathrm{~s}, 1 \mathrm{H}), 7.96(\mathrm{~d}, J=$ $8.7 \mathrm{~Hz}, 1 \mathrm{H}), 7.94(\mathrm{~s}, 1 \mathrm{H}), 7.49(\mathrm{dd}, J=8.4 \mathrm{~Hz}, 1.4 \mathrm{~Hz}, 1 \mathrm{H}), 7.26(\mathrm{~d}, J=7.7 \mathrm{~Hz}$, $2 \mathrm{H}), 7.20(\mathrm{~d}, J=7.9 \mathrm{~Hz}, 2 \mathrm{H}), 3.03-2.80(\mathrm{~m}, 4 \mathrm{H}), 2.58(\mathrm{~s}, 3 \mathrm{H}), 2.44(\mathrm{~s}, 3 \mathrm{H})$, 1.67-1.58 (m, $6 \mathrm{H}) ;{ }^{13} \mathrm{C}$ NMR (400 MHz, $\left.\mathrm{CDCl}_{3}\right) \delta 153.2,147.3,136.9,136.5$, 
$135.5,130.8,129.8,129.4,129.2,128.9,128.4,126.2,123.2,53.8,26.6,24.3$, 22.1, 21.2; HRMS m/z calcd for $\mathrm{C}_{22} \mathrm{H}_{24} \mathrm{~N}_{2}\left([\mathrm{M}+\mathrm{H}]^{\dagger}\right): 317.2012$, found 317.2010.

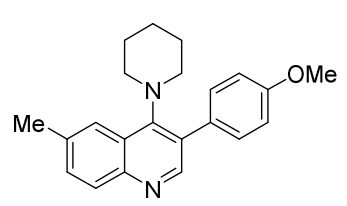

3-(4-methoxyphenyl)-6-methyl-4-(piperidin-1-yl)quinoline 8d (24 mg, yield 72\%):

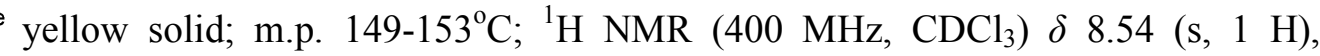
$7.98-7.94$ (m, $2 \mathrm{H}), 7.49$ (d, $J=8.2 \mathrm{~Hz}, 1 \mathrm{H}), 7.23$ (d, $J=8.5 \mathrm{~Hz}, 2 \mathrm{H}), 7.00$ (d, $J$ $=8.5 \mathrm{~Hz}, 2 \mathrm{H}), 3.89(\mathrm{~s}, 3 \mathrm{H}), 2.89-2.88(\mathrm{~m}, J=5.8 \mathrm{~Hz}, 4 \mathrm{H}), 2.58(\mathrm{~s}, 3 \mathrm{H})$, $1.68-1.59(\mathrm{~m}, 6 \mathrm{H}) ;{ }^{13} \mathrm{C} \mathrm{NMR}\left(400 \mathrm{MHz}, \mathrm{CDCl}_{3}\right) \delta 158.9,153.2,147.2,135.5$, $131.7,131.0,130.8,130.0,129.4,128.2$, 126.2, 123.2, 113.6, 55.3, 53.8, 26.6, 24.3, 22.1; HRMS m/z calcd for $\mathrm{C}_{22} \mathrm{H}_{24} \mathrm{~N}_{2} \mathrm{O}\left([\mathrm{M}+\mathrm{H}]^{+}\right)$: 332.1889, found 332.1893.

3-(4-fluorophenyl)-6-methyl-4-(piperidin-1-yl)quinoline 8e (21 mg, yield 68\%): (c) yellow solid; m.p. 132-134 ${ }^{\circ} \mathrm{C} ;{ }^{1} \mathrm{H}$ NMR (400 MHz, $\left.\mathrm{CDCl}_{3}\right) \delta 8.50$ (s, $\left.1 \mathrm{H}\right), 7.97$ $(\mathrm{d}, J=8.5 \mathrm{~Hz}, 1 \mathrm{H}), 7.93(\mathrm{~s}, 1 \mathrm{H}), 7.51(\mathrm{dd}, J=8.5 \mathrm{~Hz}, 1.5 \mathrm{~Hz}, 1 \mathrm{H}), 7.27(\mathrm{td}, J=$ $8.5 \mathrm{~Hz}, 2.2 \mathrm{~Hz}, 2 \mathrm{H}), 7.15$ (t, $J=8.6 \mathrm{~Hz}, 2 \mathrm{H}), 3.03-2.73$ (m, $4 \mathrm{H}), 2.58$ (s, $3 \mathrm{H})$, $1.68-1.59(\mathrm{~m}, 6 \mathrm{H}) ;{ }^{13} \mathrm{C} \mathrm{NMR}\left(400 \mathrm{MHz}, \mathrm{CDCl}_{3}\right) \delta 162.2(\mathrm{~d}, J(\mathrm{C}, \mathrm{F})=246.5 \mathrm{~Hz})$, 153.5, 152.7, 147.3, 135.7, 135.4, 131.5(d, J(C,F) = 7.9 Hz), 131.1, 129.4, 127.4, 126.1, 123.2, $115.2(\mathrm{~d}, J(\mathrm{C}, \mathrm{F})=21.4 \mathrm{~Hz}), 53.9,26.5,24.3,22.1 ; \mathrm{HRMS} \mathrm{m} / \mathrm{z}$ calcd for $\mathrm{C}_{21} \mathrm{H}_{21} \mathrm{FN}_{2}\left([\mathrm{M}+\mathrm{H}]^{+}\right)$: 321.1762 , found 321.1754 .

3-(4-chlorophenyl)-6-methyl-4-(piperidin-1-yl)quinoline 8 f (23 $\mathrm{mg}$, yield 70\%):

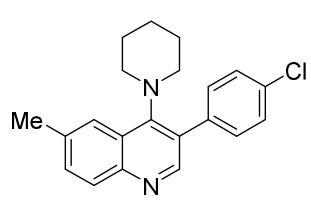

yellow solid; m.p. 103-105 ${ }^{\circ} \mathrm{C} ;{ }^{1} \mathrm{H}$ NMR $\left(400 \mathrm{MHz}, \mathrm{CDCl}_{3}\right) \delta 8.49(\mathrm{~s}, 1 \mathrm{H}), 7.96$ $(\mathrm{d}, \mathrm{J}=8.5 \mathrm{~Hz}, 1 \mathrm{H}), 7.93(\mathrm{~s}, 1 \mathrm{H}), 7.50(\mathrm{dd}, \mathrm{J}=8.5 \mathrm{~Hz}, 1.8 \mathrm{~Hz}, 1 \mathrm{H}), 7.43(\mathrm{~d}, \mathrm{~J}=$ $8.3 \mathrm{~Hz}, 2 \mathrm{H}), 7.24$ (d, J = 8.3 Hz, $2 \mathrm{H}), 3.10-2.68$ (m, $4 \mathrm{H}), 2.58$ (s, $3 \mathrm{H}), 1.70-1.60$ $(\mathrm{m}, 6 \mathrm{H}) ;{ }^{13} \mathrm{C} \mathrm{NMR}\left(400 \mathrm{MHz}, \mathrm{CDCl}_{3}\right) \delta 153.4,152.5,147.5,138.0,135.7,133.4$, 131.2, 129.5, 128.4, 127.1, 126.0, 123.2, 54.0, 26.5, 24.3, 22.1; HRMS m/z calcd for $\mathrm{C}_{21} \mathrm{H}_{21} \mathrm{ClN}_{2}\left([\mathrm{M}+\mathrm{H}]^{+}\right): 337.1466$, found 337.1465 .

3-(4-bromophenyl)-6-methyl-4-(piperidin-1-yl)quinoline 8g (26 mg, yield 69\%):

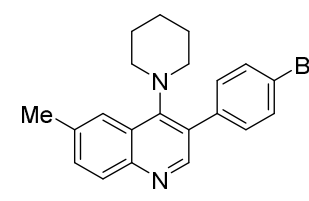

Br yellow solid; m.p. $148-149{ }^{\circ} \mathrm{C} ;{ }^{1} \mathrm{H}$ NMR (400 MHz, $\left.\mathrm{CDCl}_{3}\right) \delta 8.49$ (s, $\left.1 \mathrm{H}\right), 7.96$ $(\mathrm{d}, J=8.5 \mathrm{~Hz}, 1 \mathrm{H}), 7.93(\mathrm{~s}, 1 \mathrm{H}), 7.59$ (d, $J=8.3 \mathrm{~Hz}, 2 \mathrm{H}), 7.50$ (dd, $J=8.5 \mathrm{~Hz}$, $1.2 \mathrm{~Hz}, 1 \mathrm{H}), 7.19$ (d, $J=8.3 \mathrm{~Hz}, 2 \mathrm{H}), 2.94-2.74(\mathrm{~m}, 4 \mathrm{H}), 2.58$ (s, $3 \mathrm{H}), 1.68-1.60$ $(\mathrm{m}, 6 \mathrm{H}) ;{ }^{13} \mathrm{C} \mathrm{NMR}\left(400 \mathrm{MHz}, \mathrm{CDCl}_{3}\right) \delta 153.4,152.5,147.5,138.5,135.8,131.6$, 131.4, 131.2, 129.5, 127.1, 126.1, 123.2, 121.5, 54.0, 26.6, 24.3, 22.2; HRMS m/z calcd for $\mathrm{C}_{21} \mathrm{H}_{21} \mathrm{BrN}_{2}\left([\mathrm{M}+\mathrm{H}]^{+}\right)$: 381.0961, found 381.0963.

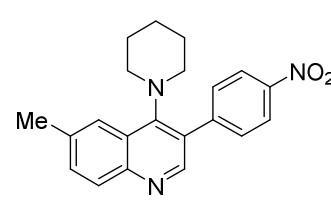

6-methyl-3-(4-nitrophenyl)-4-(piperidin-1-yl)quinoline $\mathbf{8 h}$ (15 mg, yield 44\%): yellow solid; m.p. $185-188{ }^{\circ} \mathrm{C} ;{ }^{1} \mathrm{H}$ NMR $\left(400 \mathrm{MHz}, \mathrm{CDCl}_{3}\right) \delta 8.50(\mathrm{~s}, 1 \mathrm{H}), 8.34$ (d, $J=8.8 \mathrm{~Hz}, 2 \mathrm{H}), 7.99-7.94$ (m, $2 \mathrm{H}), 7.56-7.48$ (m, $3 \mathrm{H}), 3.01-2.82$ (m, $4 \mathrm{H})$, 2.59 (s, $3 \mathrm{H}), 1.71-1.62(\mathrm{~m}, 6 \mathrm{H}) ;{ }^{13} \mathrm{C} \mathrm{NMR}\left(400 \mathrm{MHz}, \mathrm{CDCl}_{3}\right) \delta 153.7,151.8$, 
148.0, 147.1, 146.7, 136.1, 131.7, 130.7, 130.2, 129.7, 125.9, 123.6, 123.2, 54.3, 26.5, 24.2, 22.2; HRMS m/z calcd for $\mathrm{C}_{21} \mathrm{H}_{21} \mathrm{~N}_{3} \mathrm{O}_{2}\left([\mathrm{M}+\mathrm{H}]^{+}\right): 348.1707$, found 348.1727 .

1-(4-(6-methyl-4-(piperidin-1-yl)quinolin-3-yl)phenyl)ethan-1-one $\quad 8 \mathbf{i} \quad(25 \mathrm{mg}$, (1) $J=8.3 \mathrm{~Hz}, 2 \mathrm{H}), 7.98$ (d, $J=8.5 \mathrm{~Hz}, 1 \mathrm{H}), 7.93$ (s, $1 \mathrm{H}), 7.52$ (dd, $J=8.5 \mathrm{~Hz}, 1.8$ Hz, 3 H), 7.42 (d, J=8.3 Hz, 2 H), 2.94-2.88 (m, 4 H), 2.68 (s, 3 H), 2.59 (s, 3 H), 1.70-1.60 (m, $6 \mathrm{H}) ;{ }^{13} \mathrm{C}$ NMR (400 MHz, $\left.\mathrm{CDCl}_{3}\right) \delta 197.8,160.9,153.8,152.0$, $147.3,144.6,136.0,131.5,130.2,129.3,128.4,127.0,126.0,123.3,54.1,29.7$, 26.5, 24.3, 22.2; HRMS m/z calcd for $\mathrm{C}_{23} \mathrm{H}_{24} \mathrm{~N}_{2} \mathrm{O}\left([\mathrm{M}+\mathrm{H}]^{+}\right)$: 345.1961, found 345.1959 .

3-cyclopropyl-6-methyl-4-(piperidin-1-yl)quinoline 8j (18 mg, yield 68\%): yellow oil; ${ }^{1} \mathrm{H}$ NMR (400 MHz, $\left.\mathrm{CDCl}_{3}\right) \delta 8.52(\mathrm{~s}, 1 \mathrm{H}), 7.92(\mathrm{~s}, 1 \mathrm{H}), 7.90(\mathrm{~d}, J=8.9 \mathrm{~Hz}$, $1 \mathrm{H}), 7.42(\mathrm{dd}, J=8.5 \mathrm{~Hz}, 1.3 \mathrm{~Hz}, 1 \mathrm{H}), 3.42-3.40(\mathrm{~m}, 4 \mathrm{H}), 2.55(\mathrm{~s}, 3 \mathrm{H})$, 2.16-2.12 (m, $1 \mathrm{H}), 1.81-1.75(\mathrm{~m}, 6 \mathrm{H}), 1.03(\mathrm{~d}, J=7.4 \mathrm{~Hz}, 2 \mathrm{H}), 0.78(\mathrm{~d}, J=5.1$ $\mathrm{Hz}, 2 \mathrm{H}) ;{ }^{13} \mathrm{C}$ NMR $\left(400 \mathrm{MHz}, \mathrm{CDCl}_{3}\right) \delta 155.9,151.3,146.8,135.4,130.5$, 129.2, 129.0, 126.5, 123.0, 52.6, 27.0, 24.6, 22.1, 12.4, 8.1; HRMS m/z calcd for $\mathrm{C}_{18} \mathrm{H}_{22} \mathrm{~N}_{2}\left([\mathrm{M}+\mathrm{H}]^{+}\right): 267.1856$, found 267.1846.

\section{X-Ray Diffraction Structure of Compound 8a}

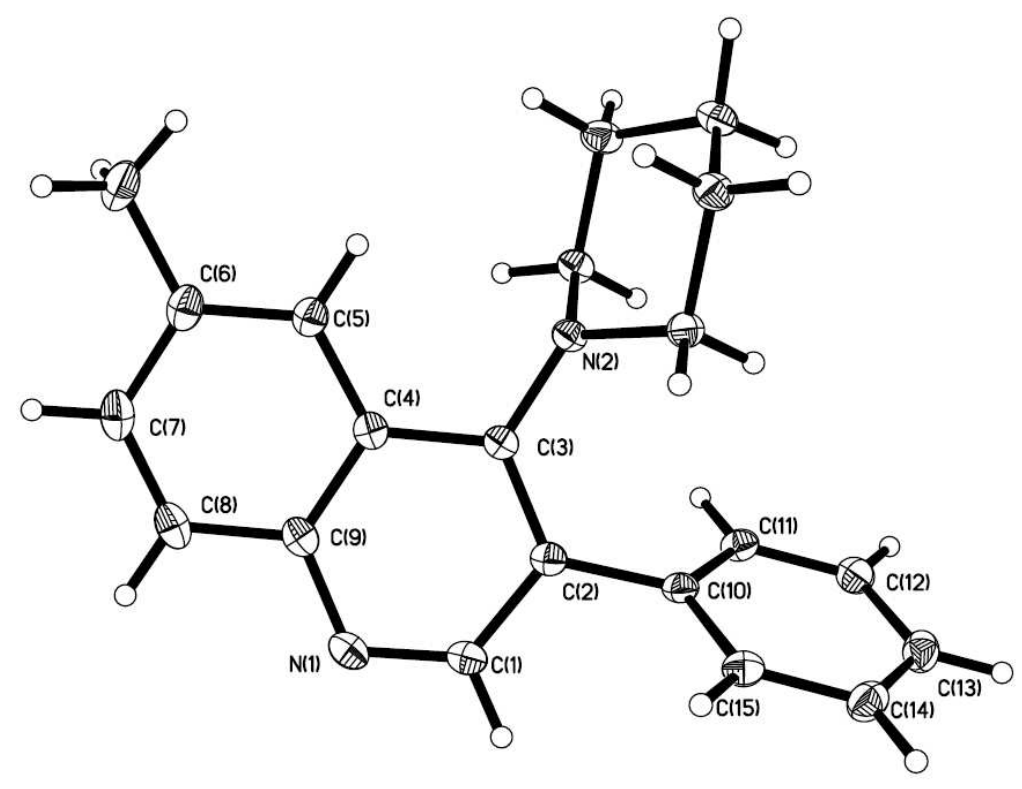




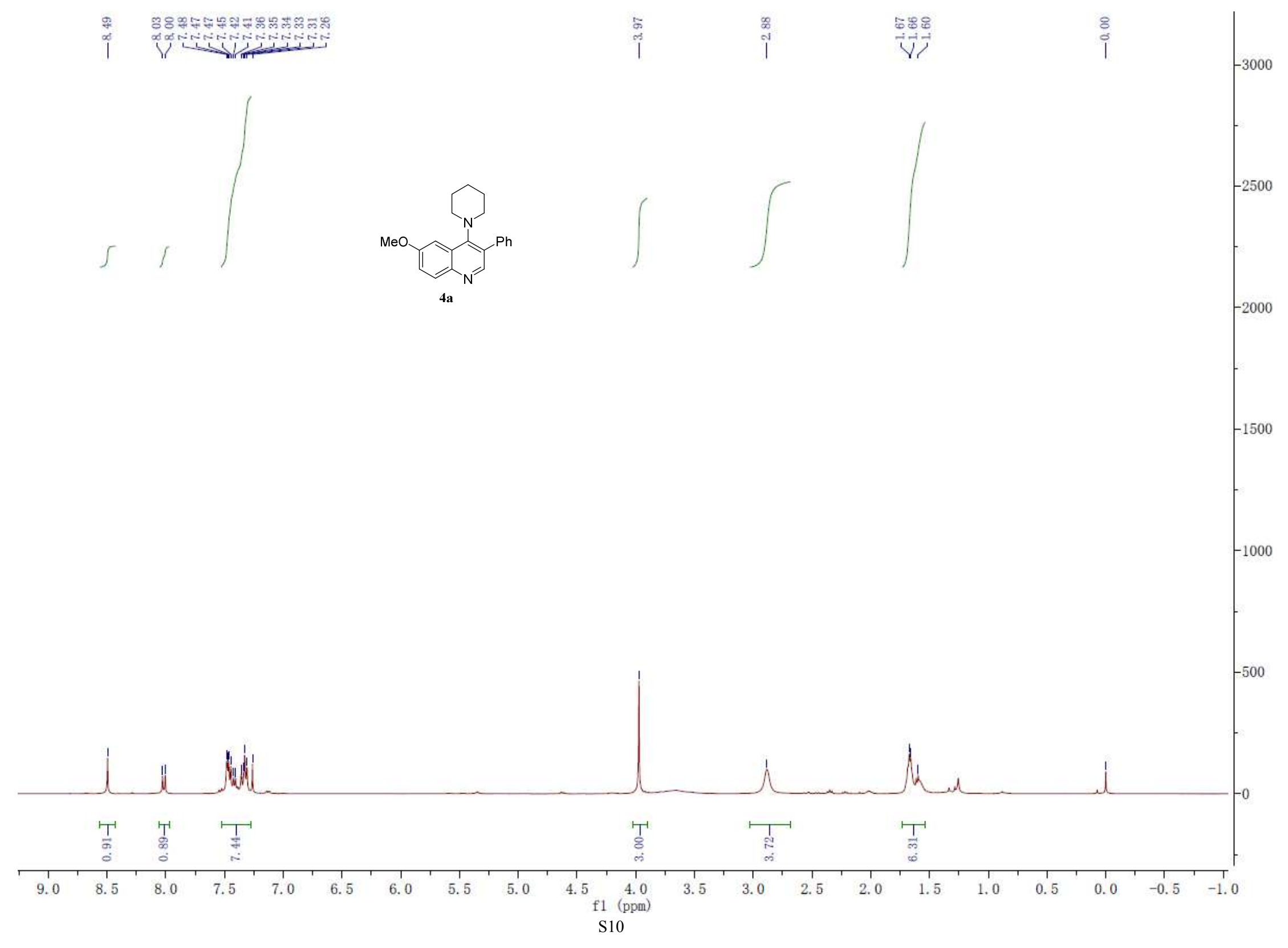




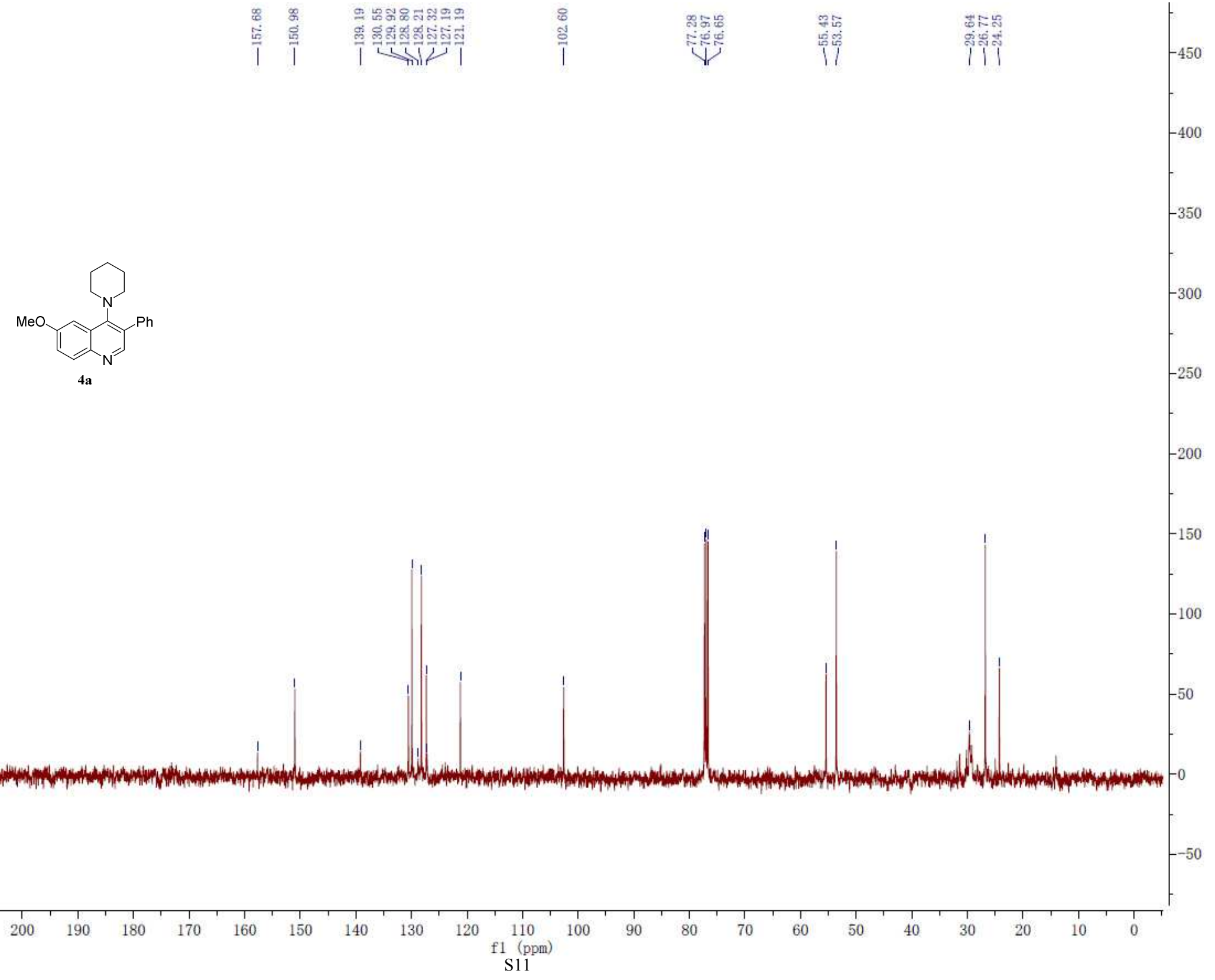




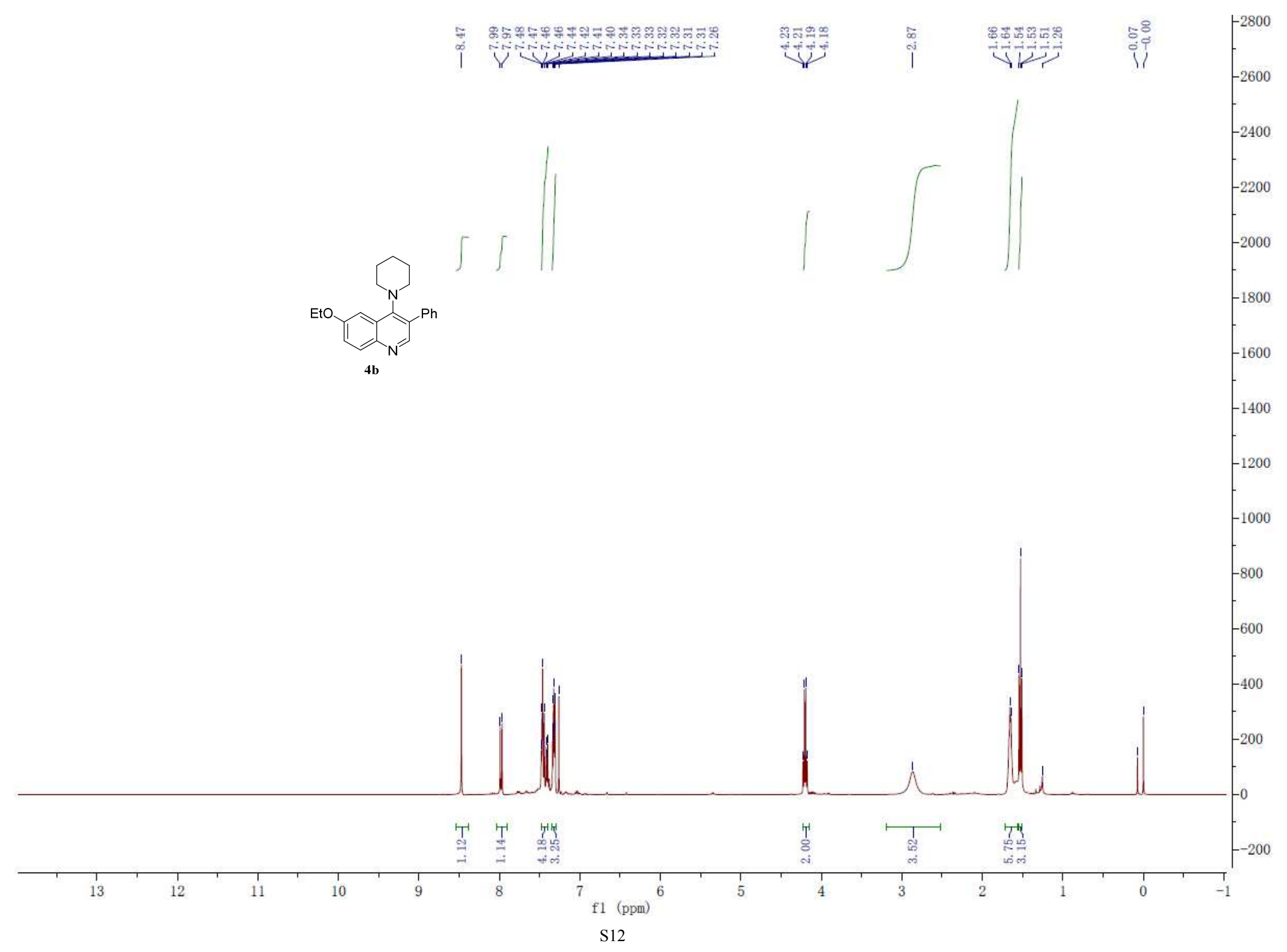




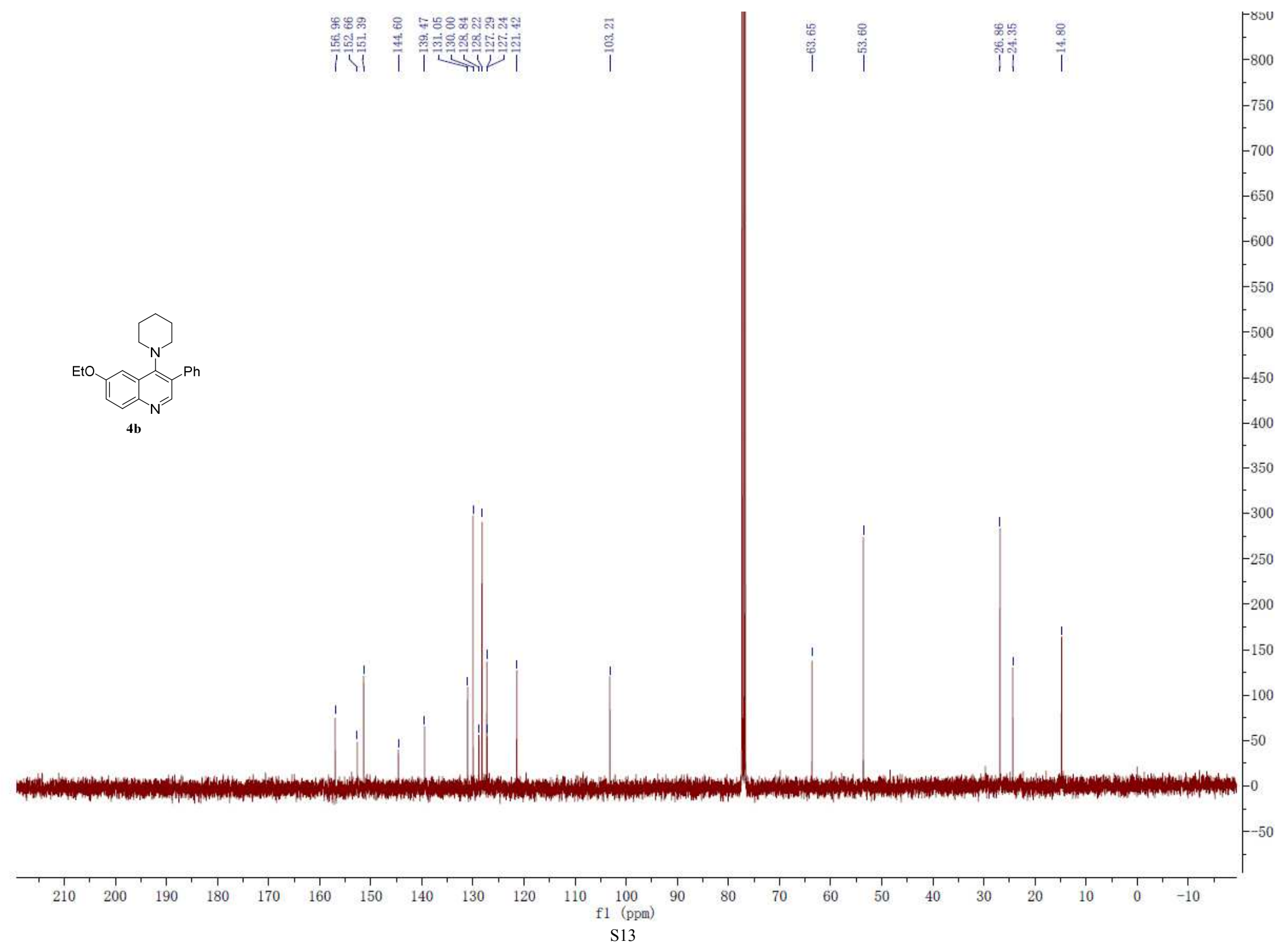




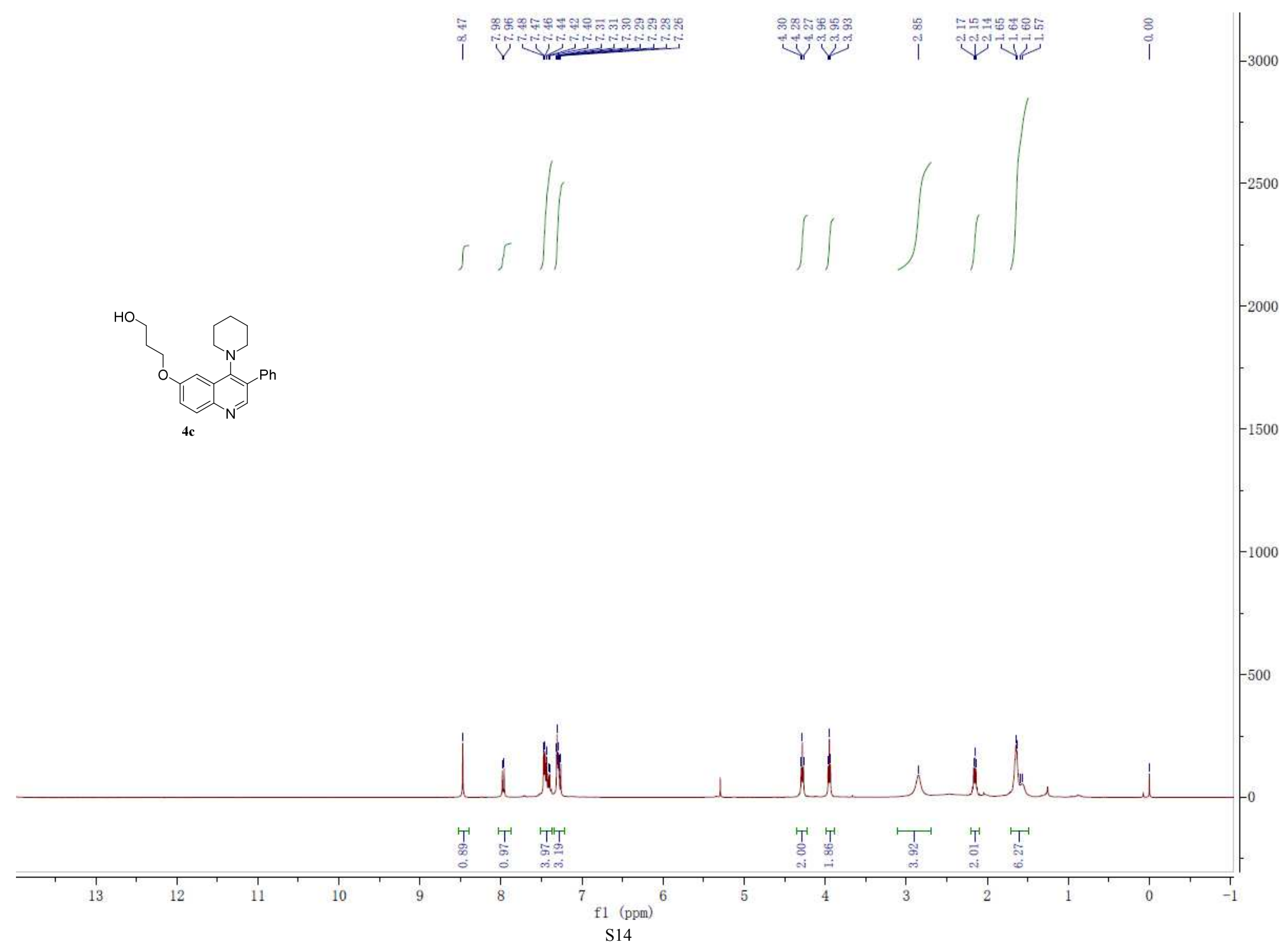




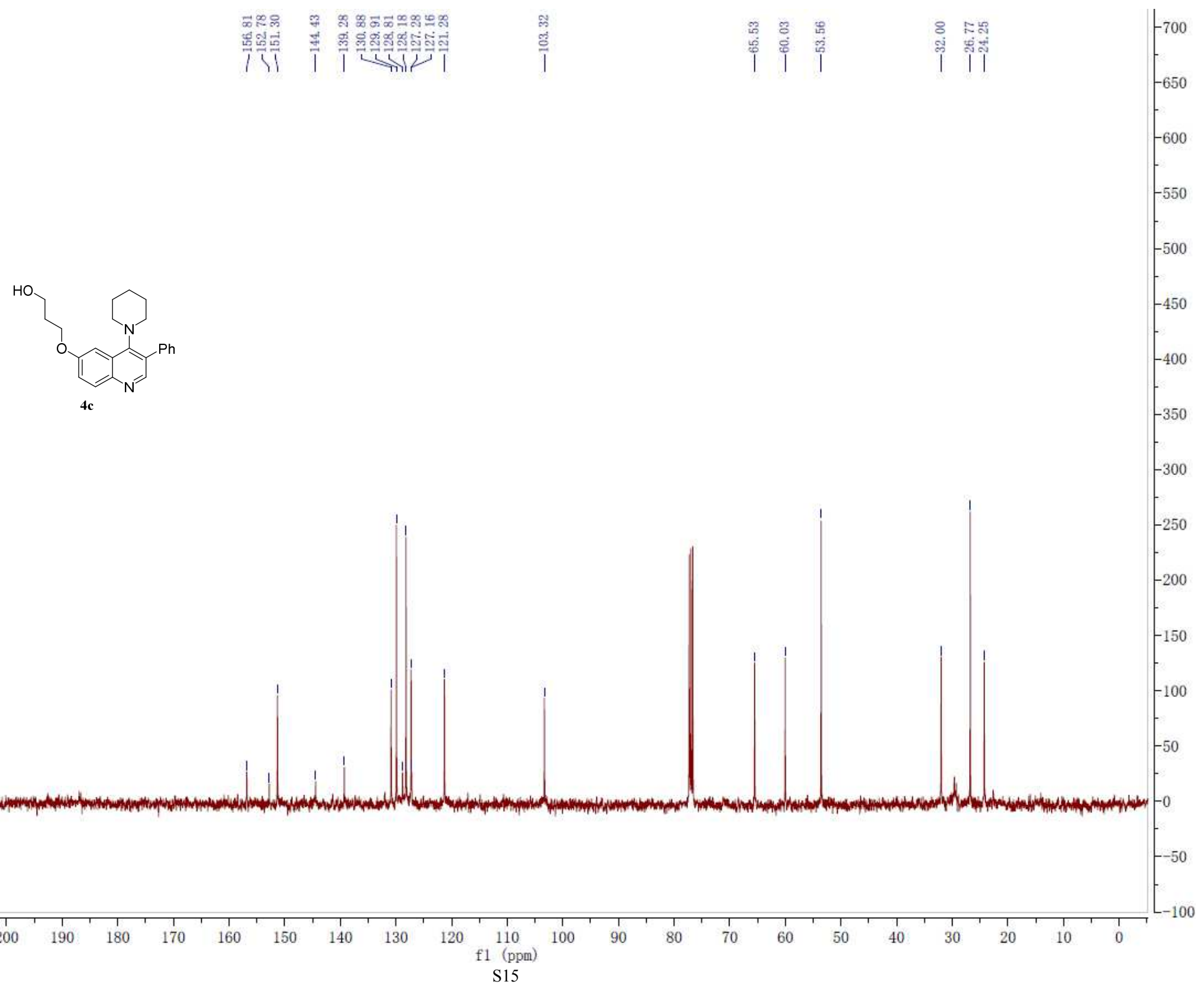




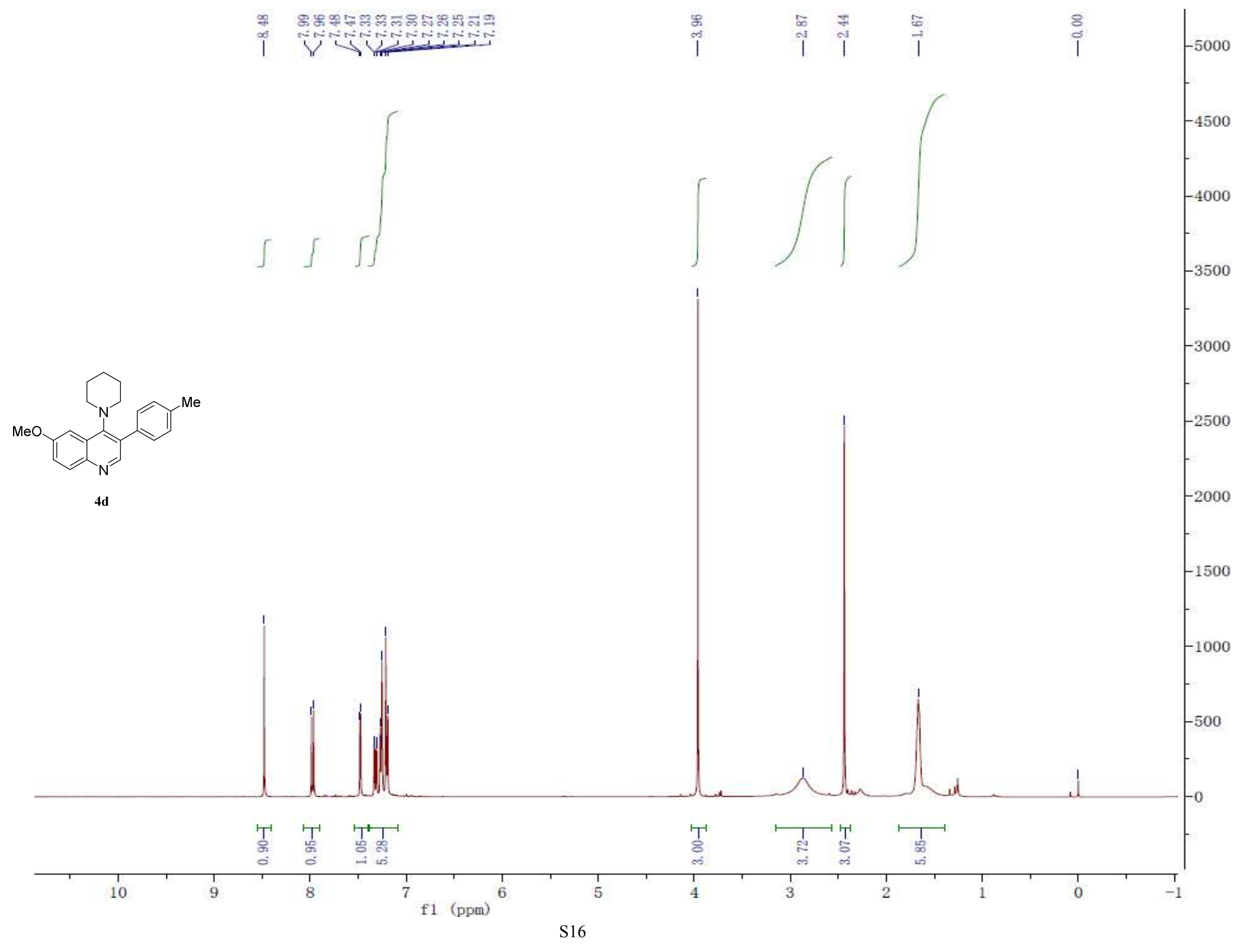




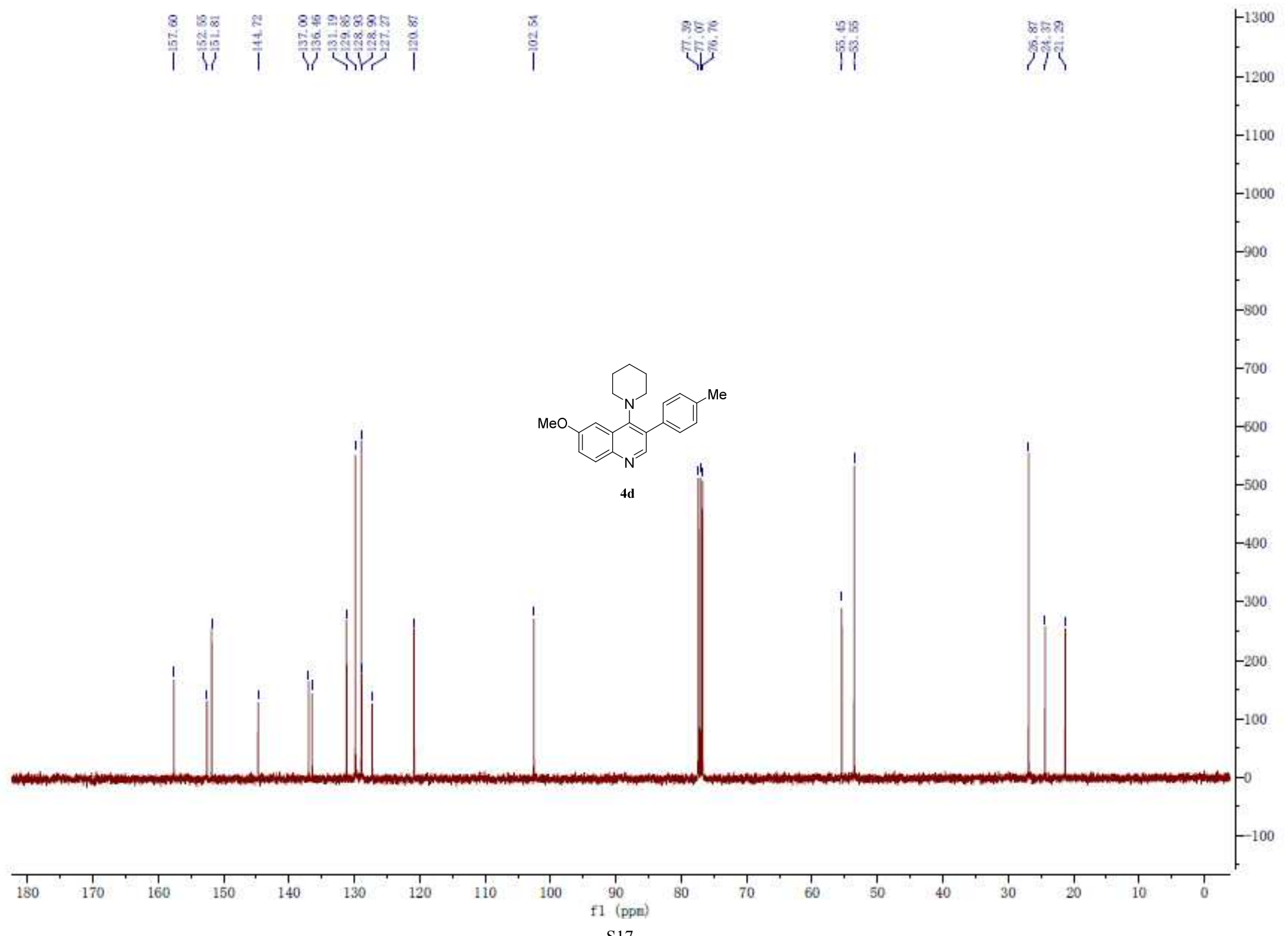

S17 


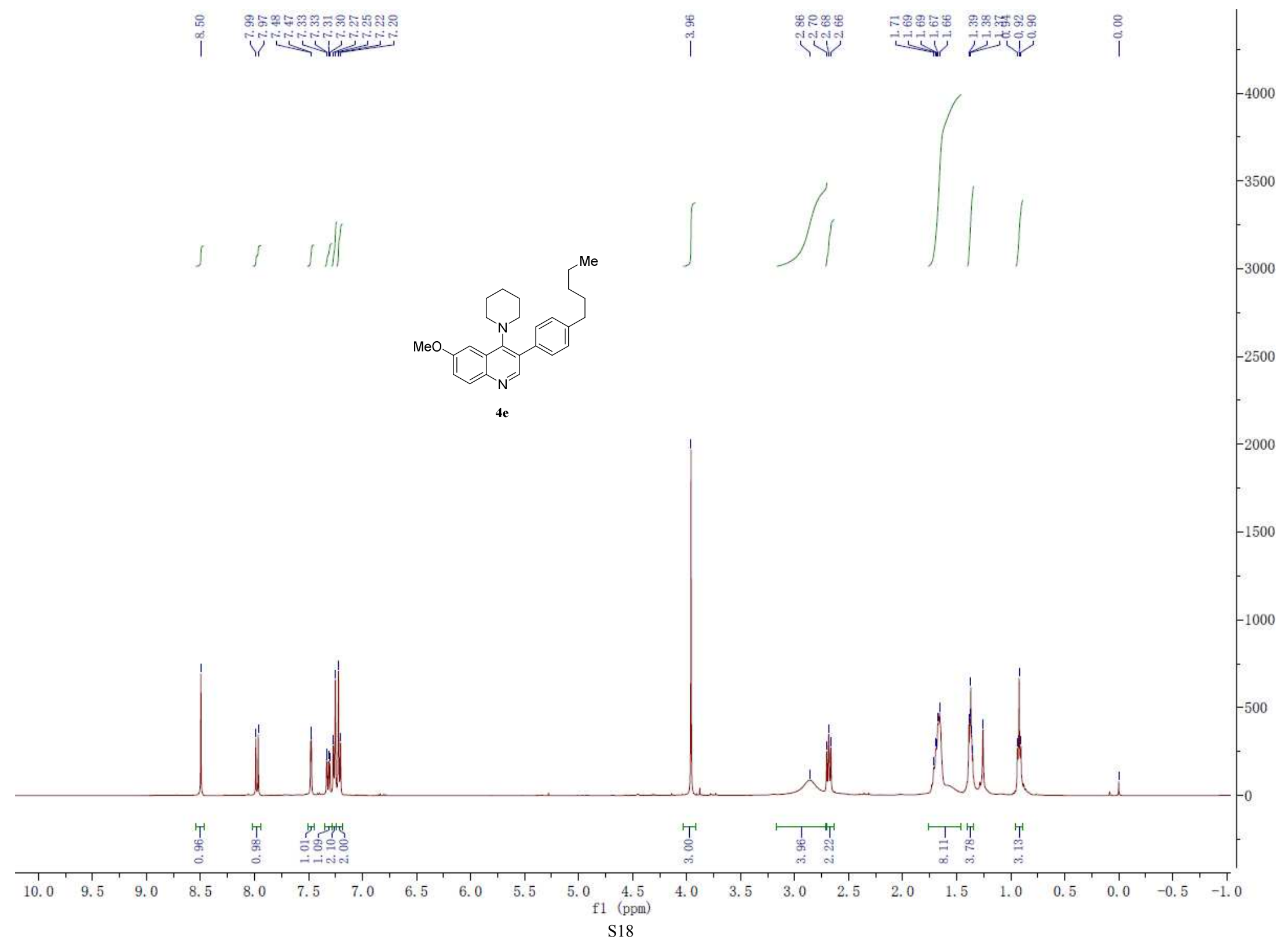




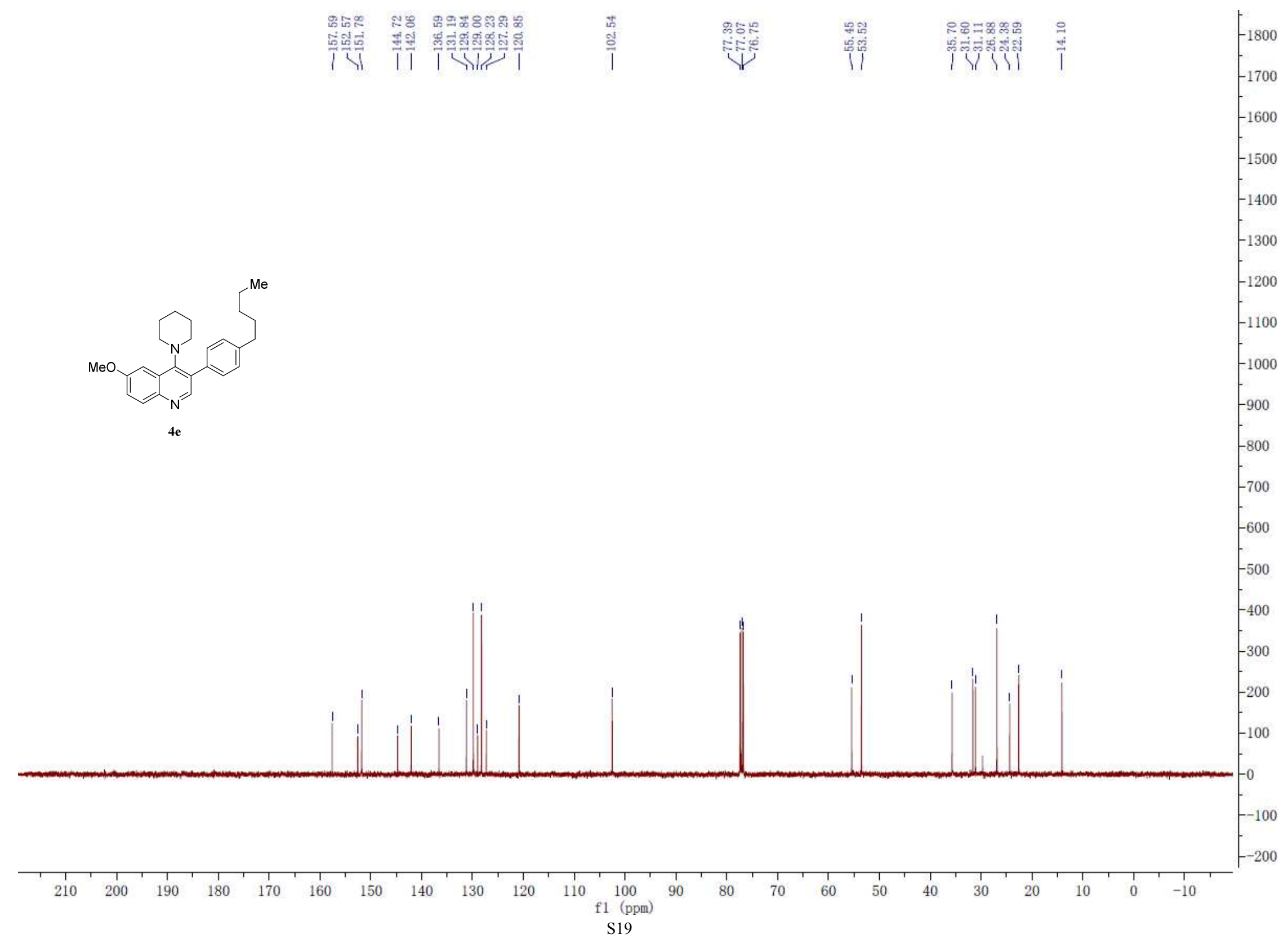




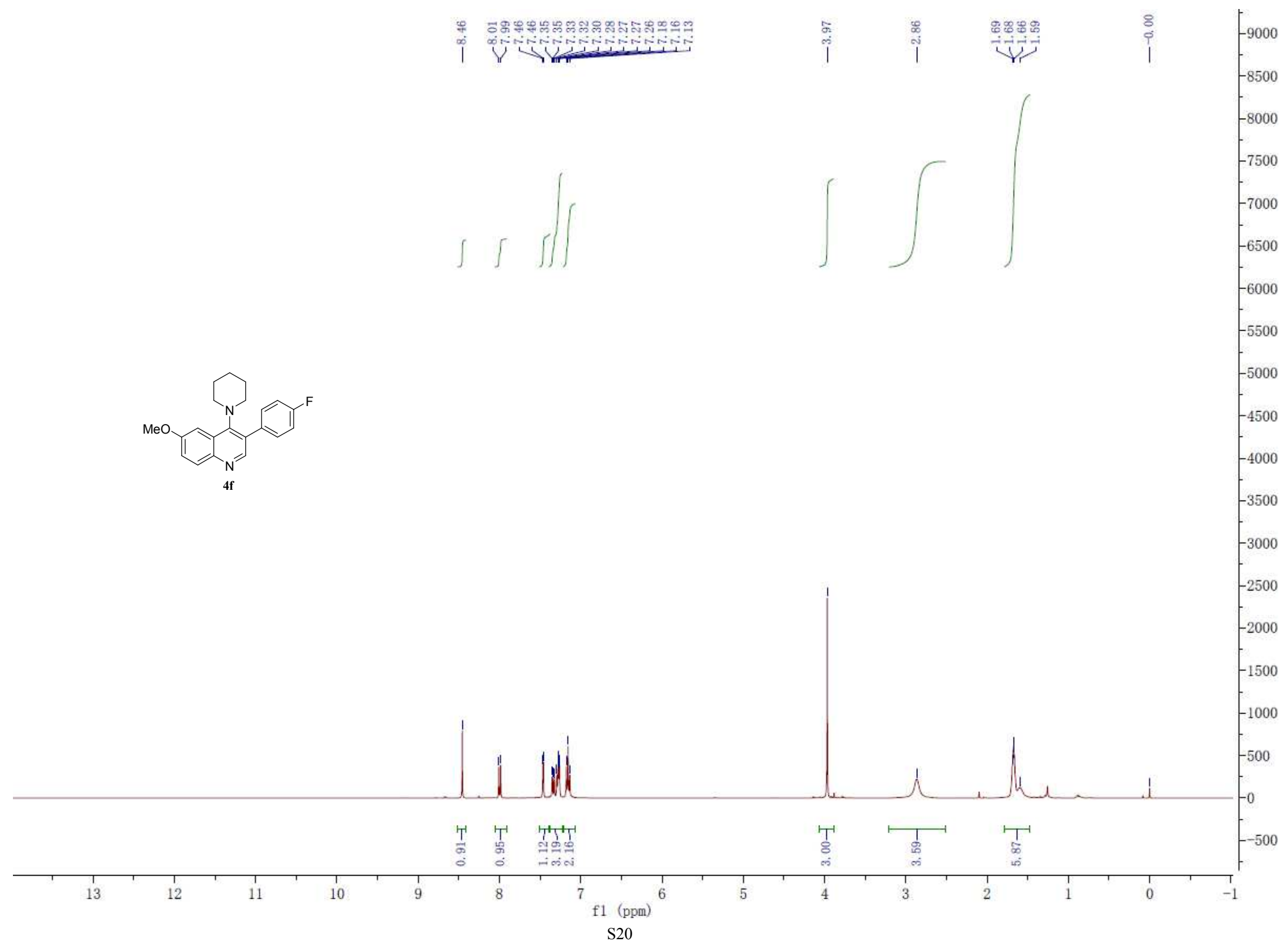




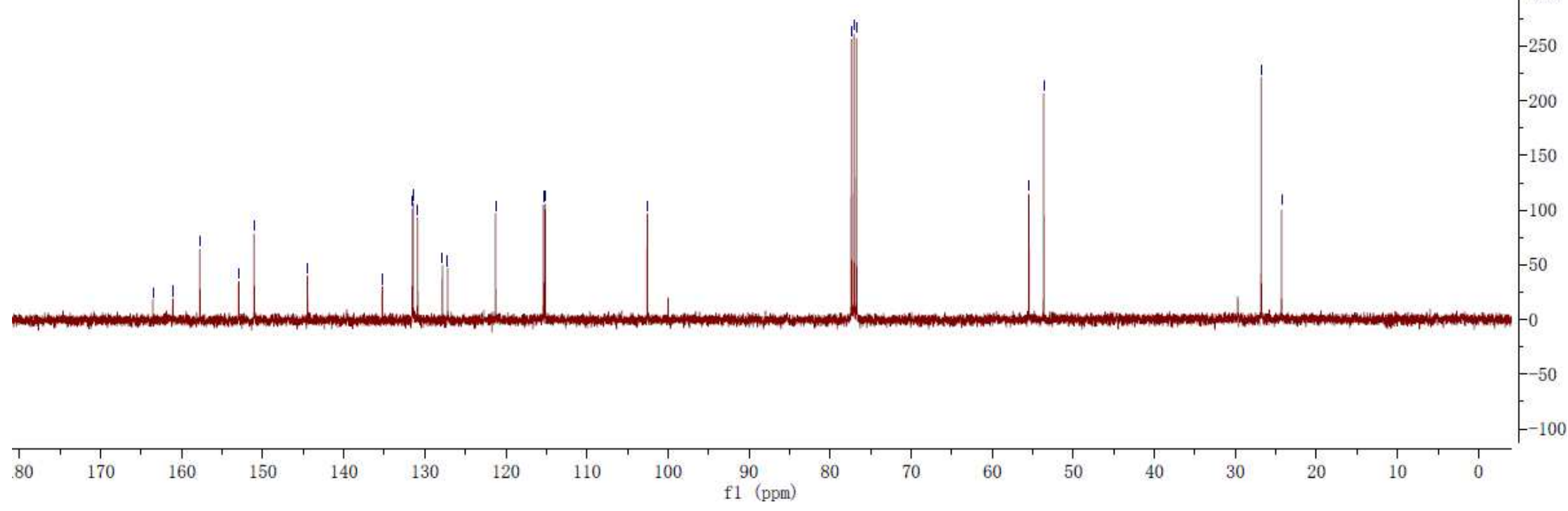




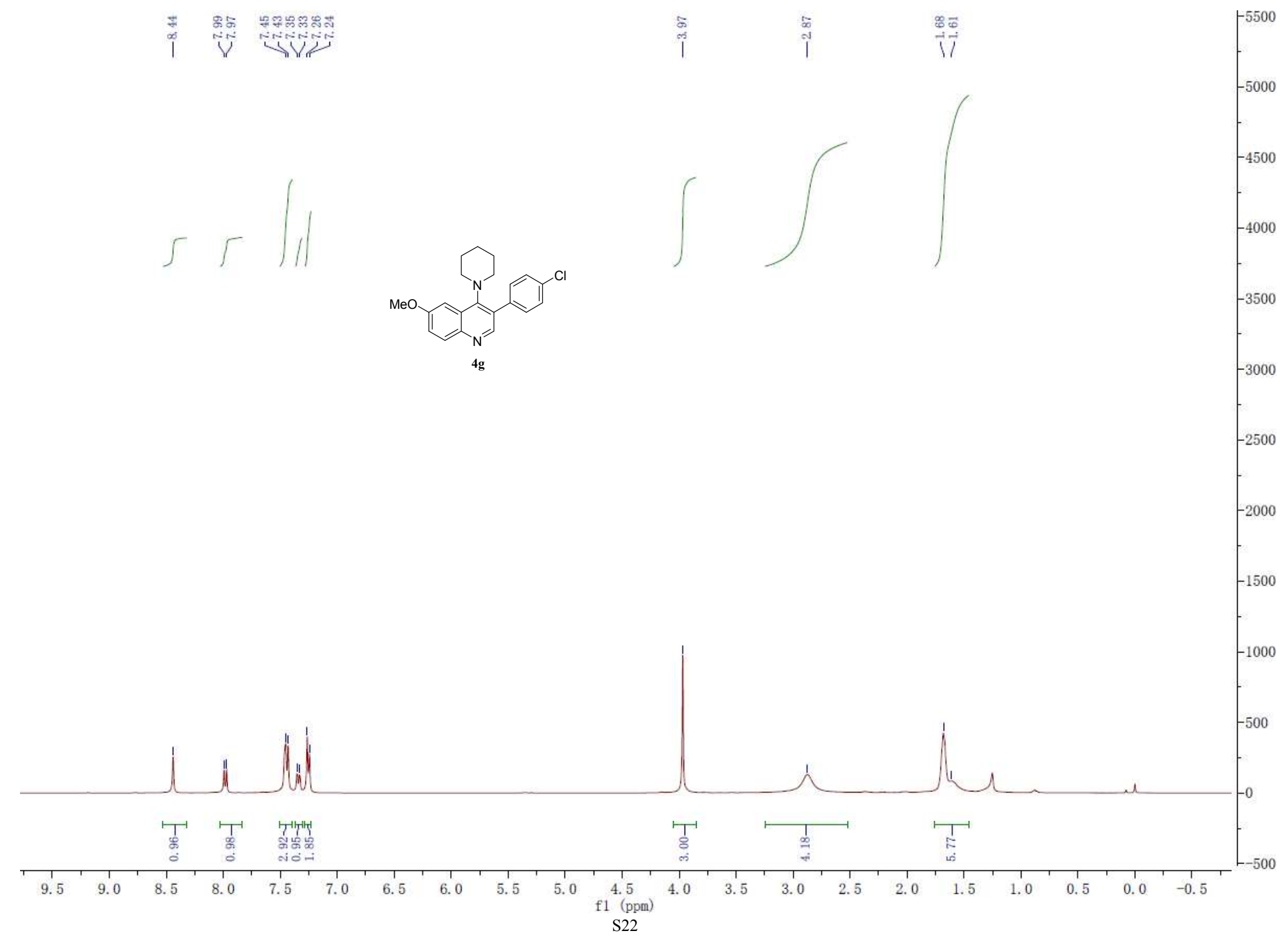




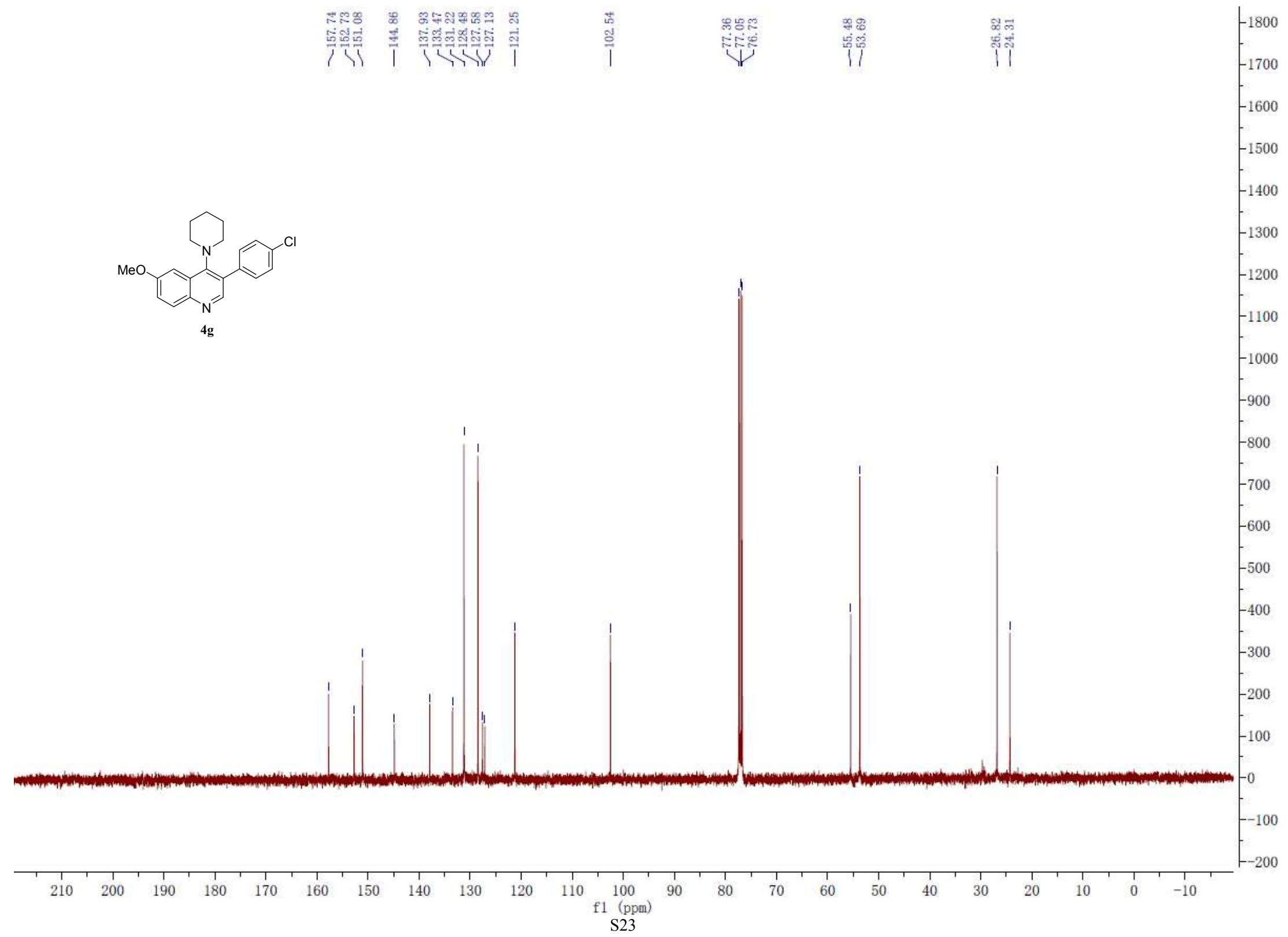




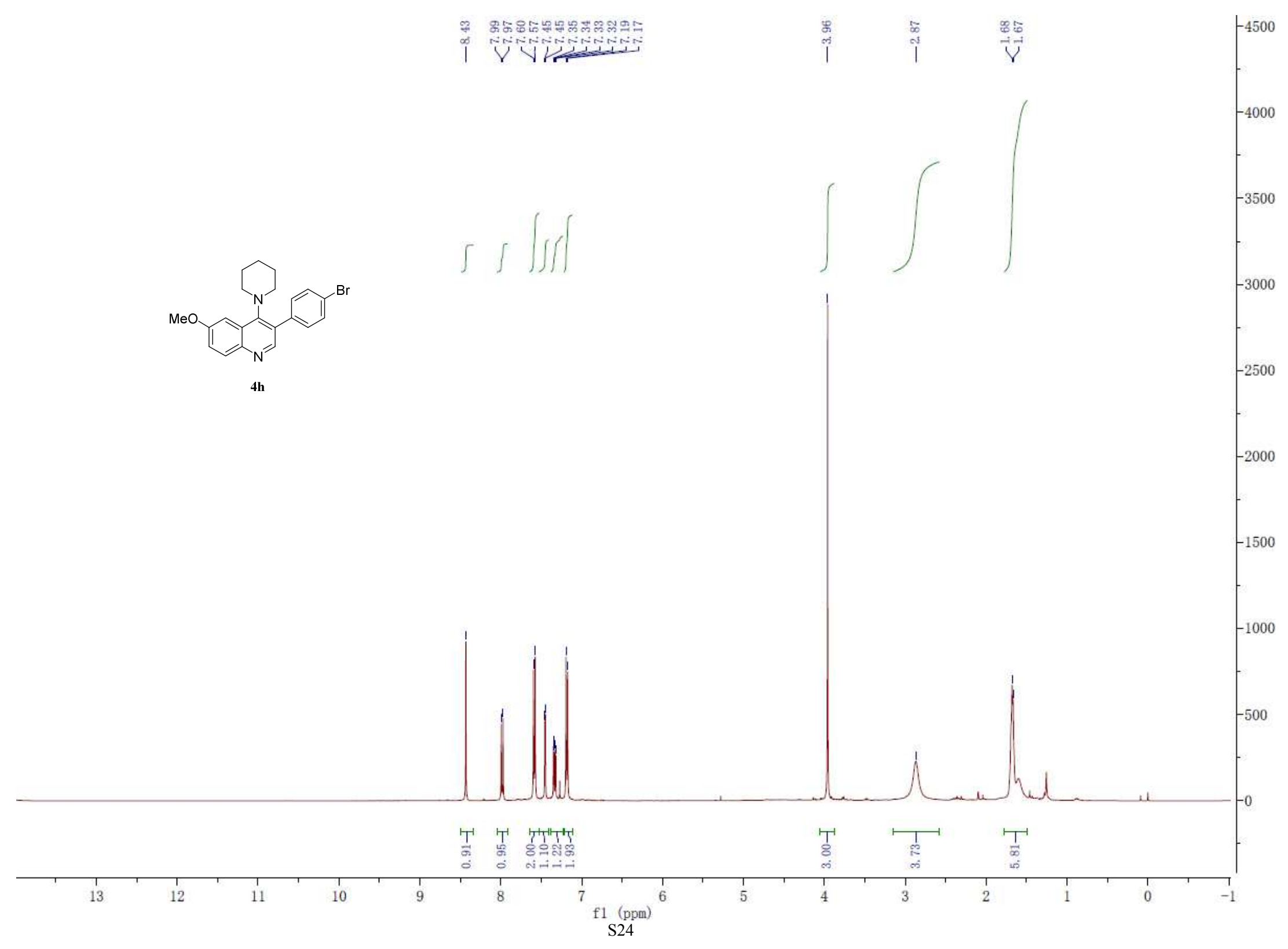




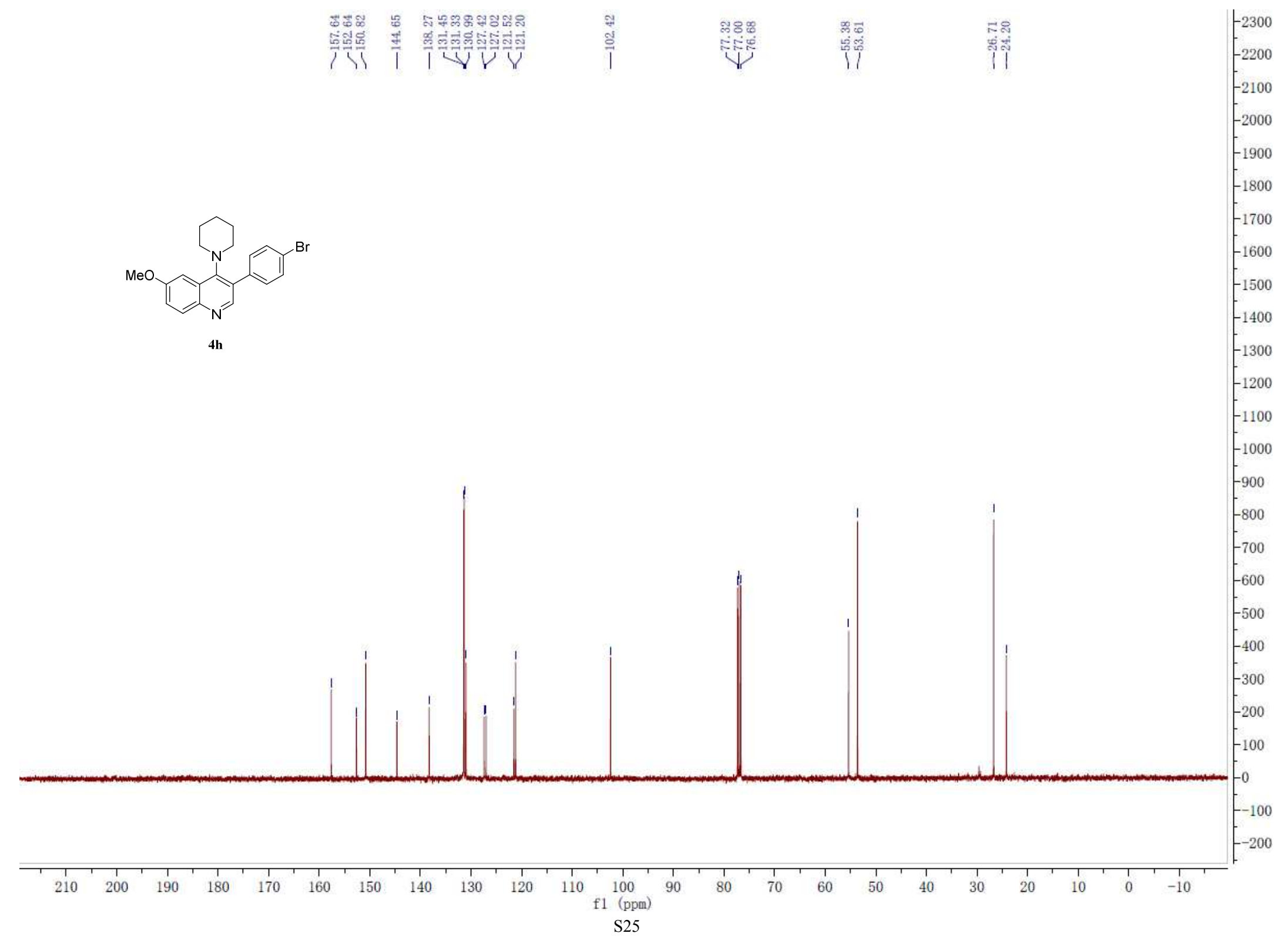




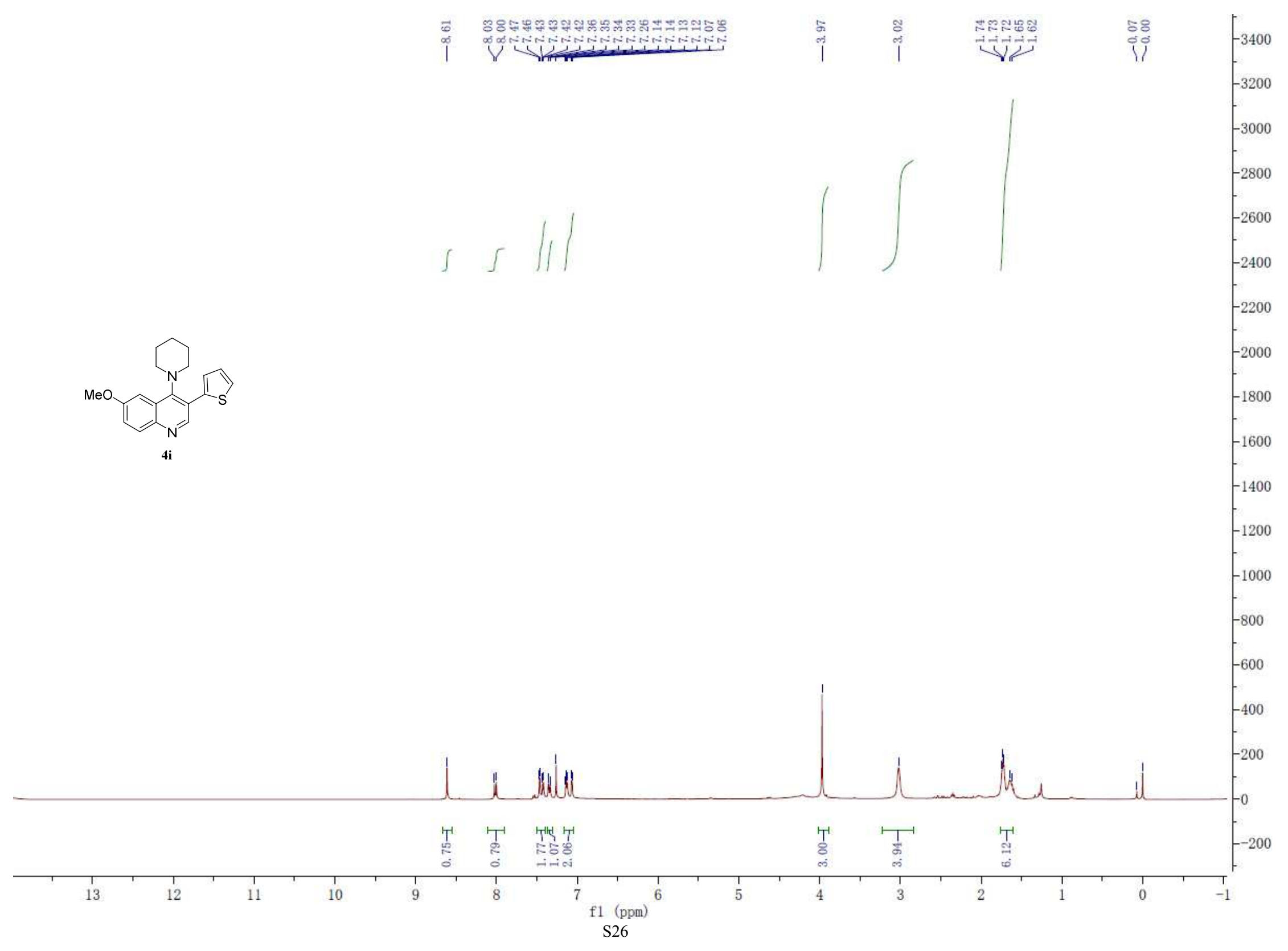




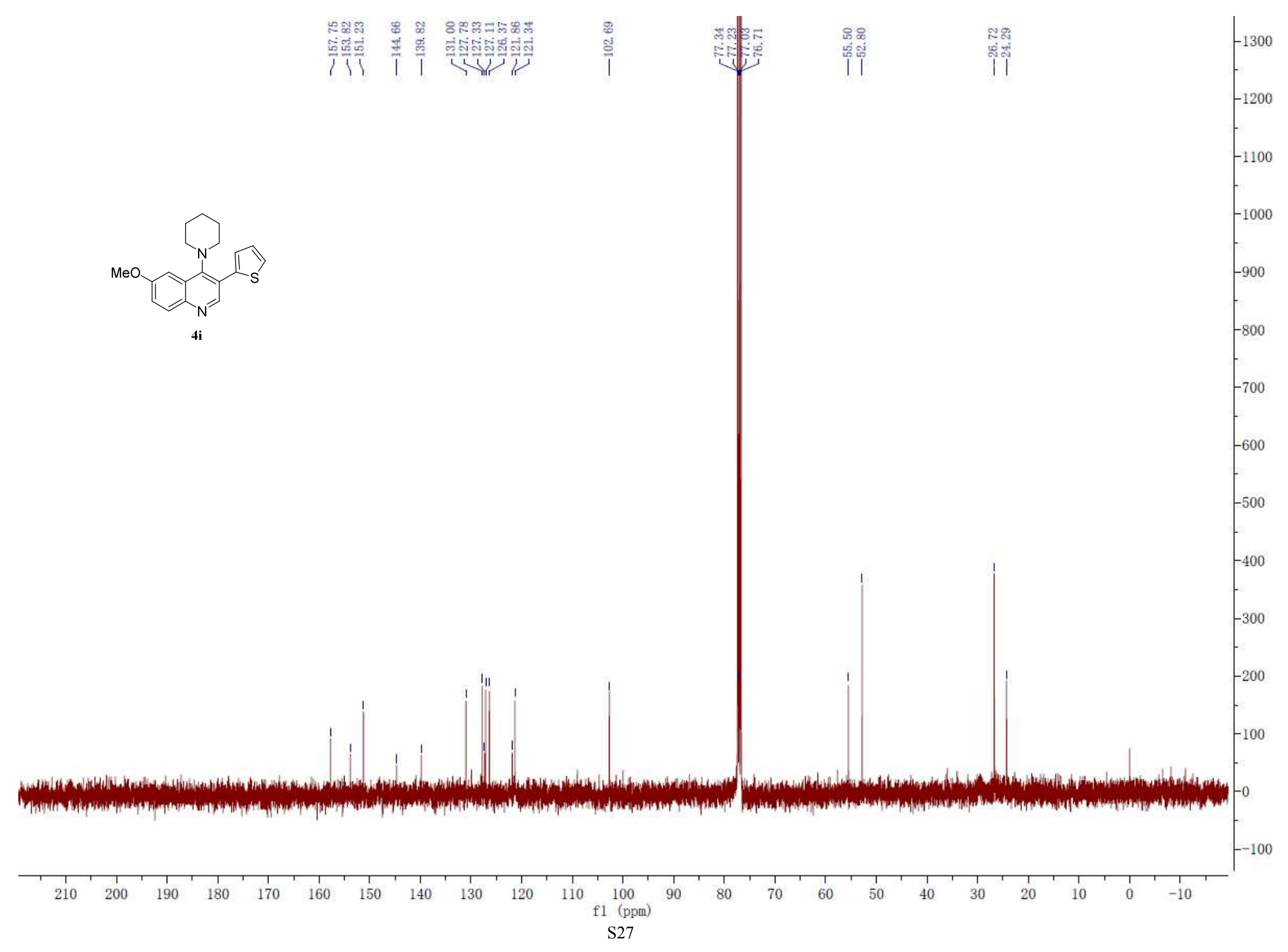




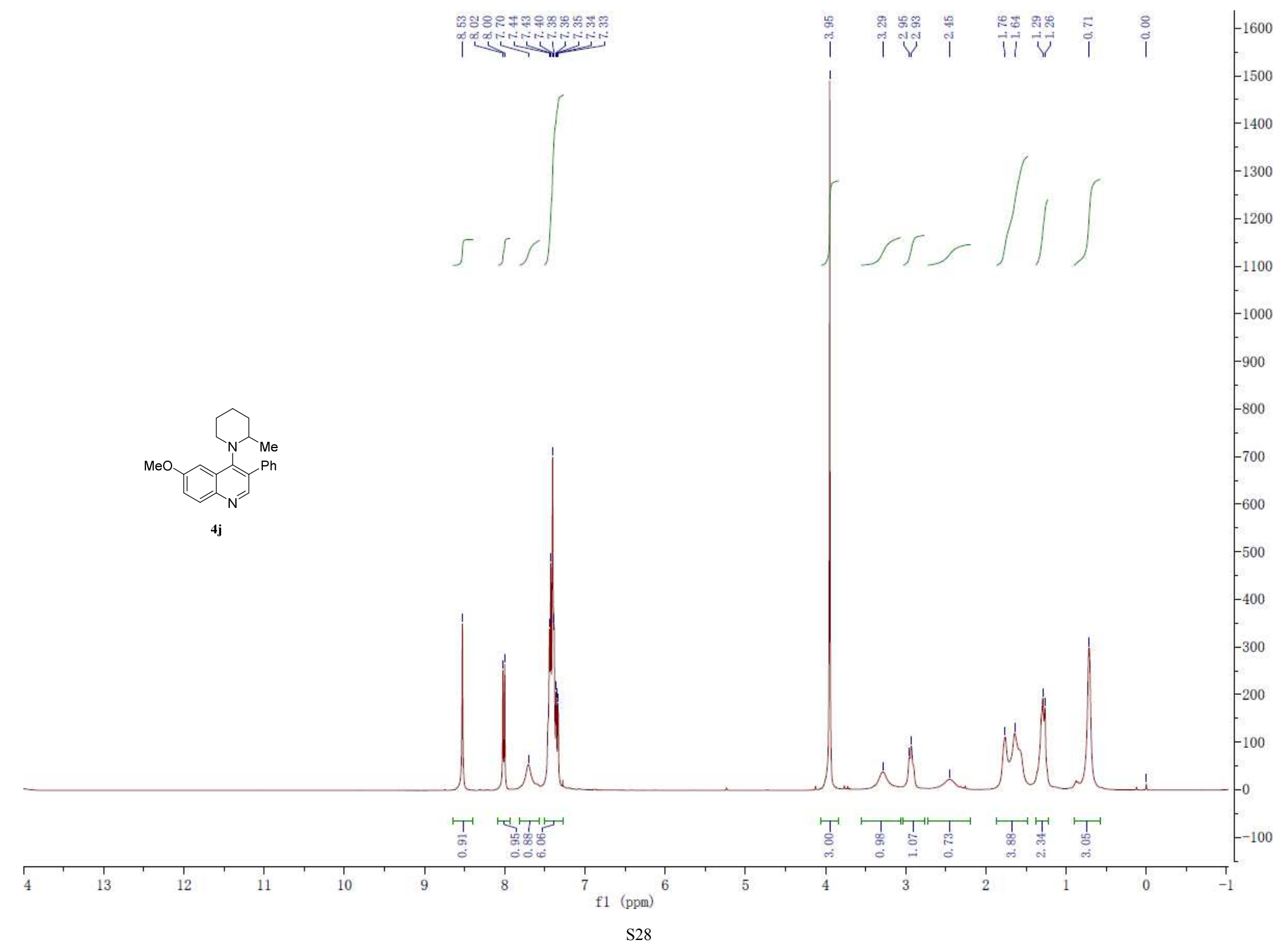




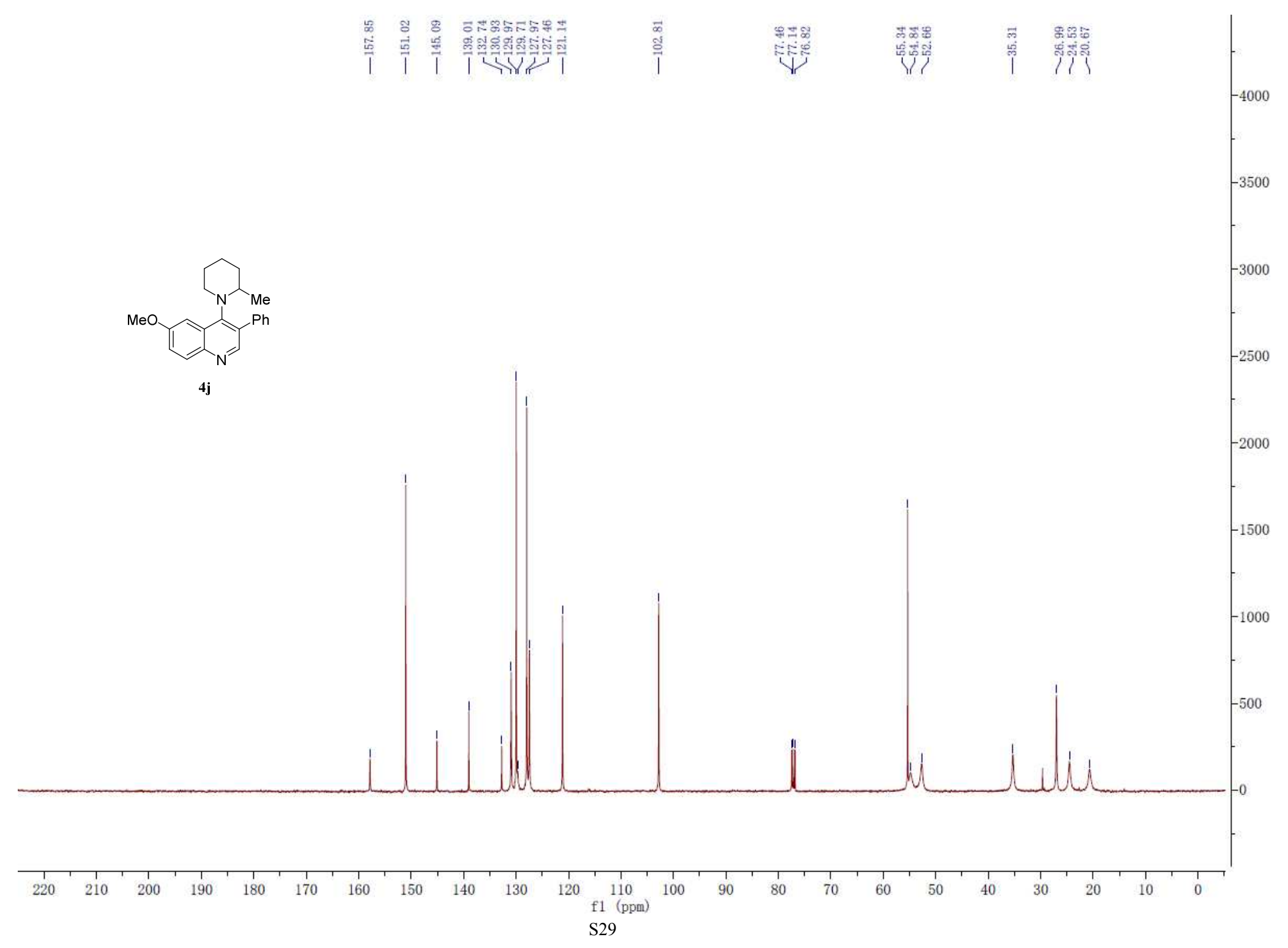




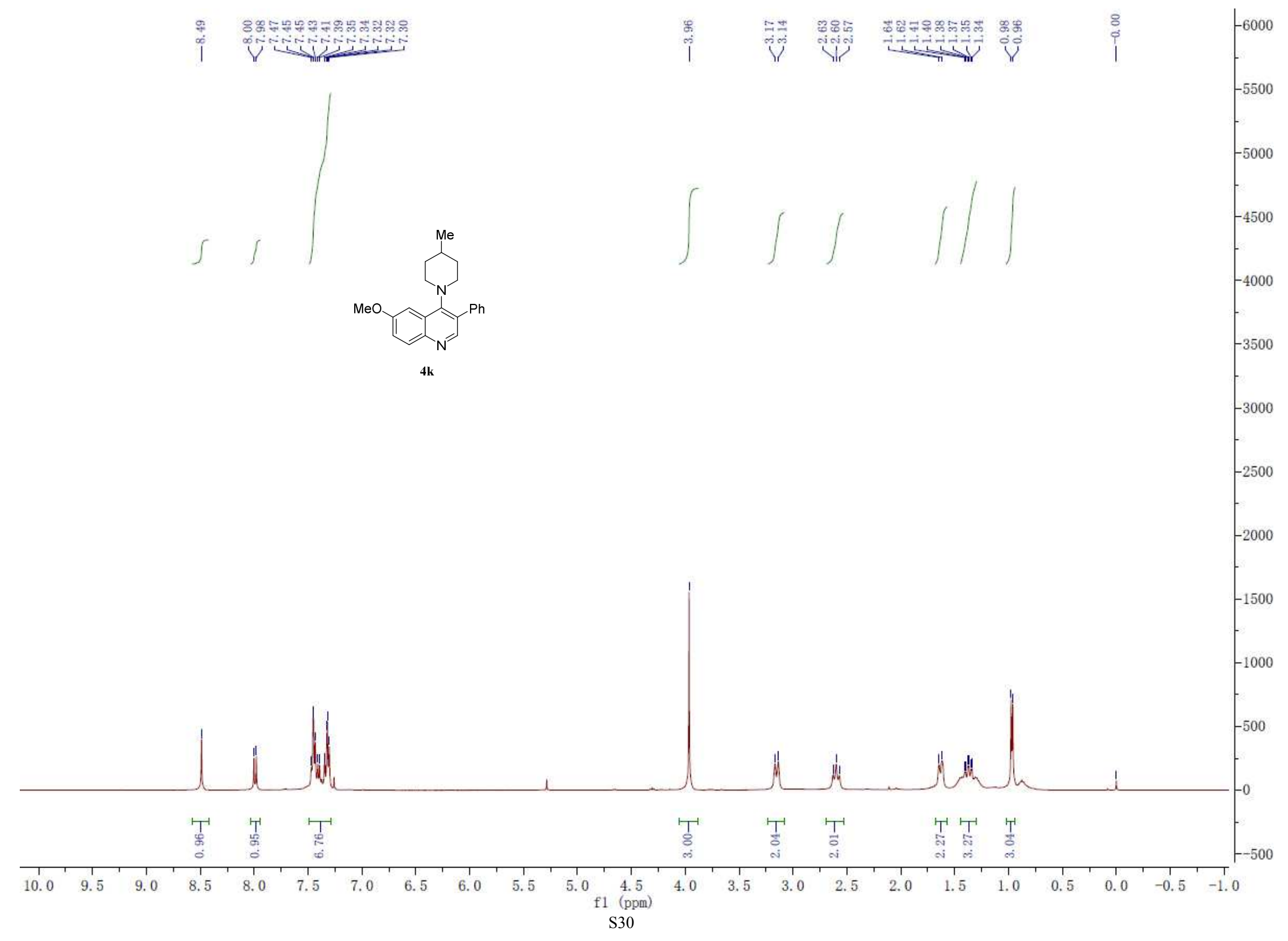




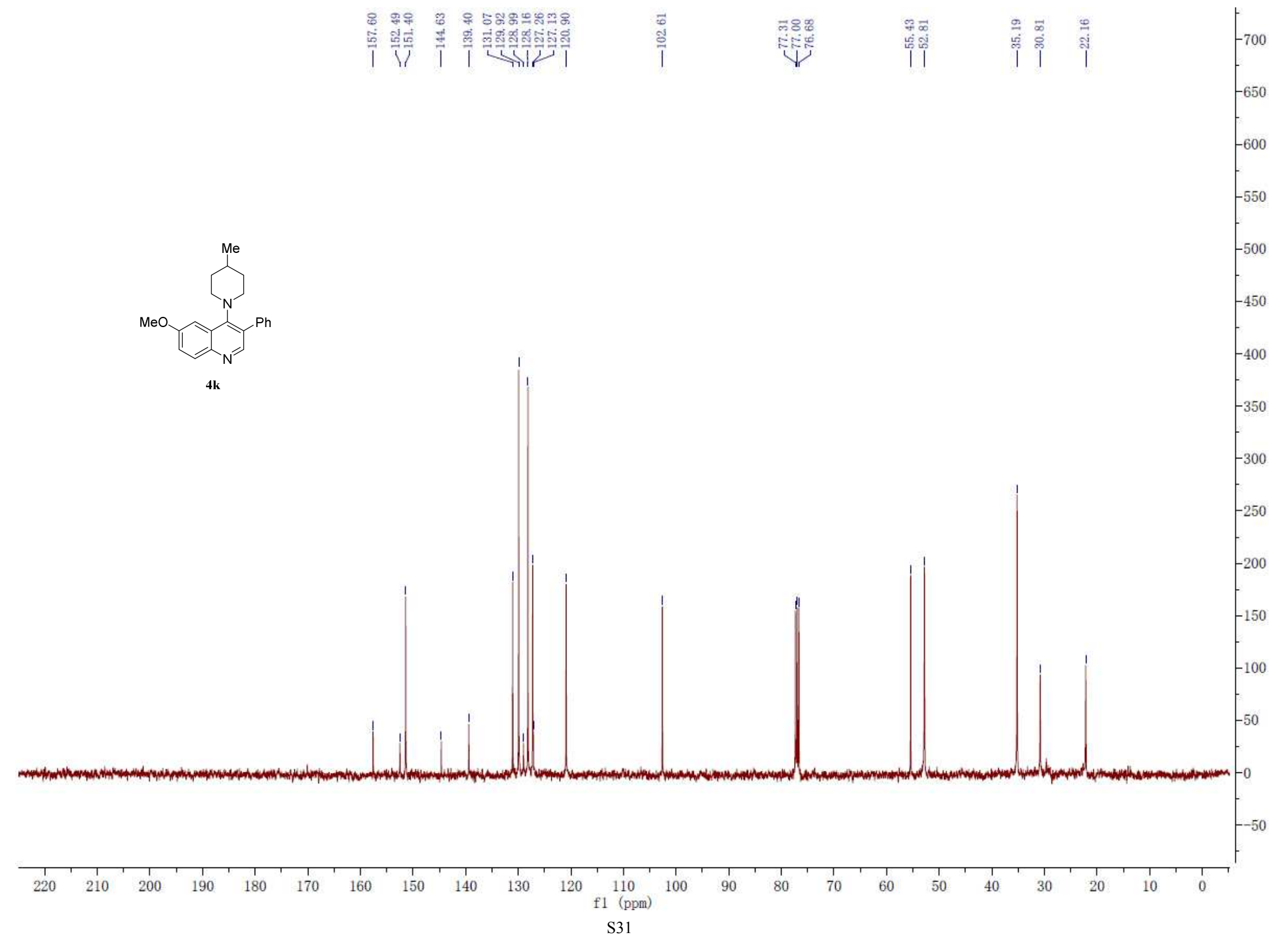




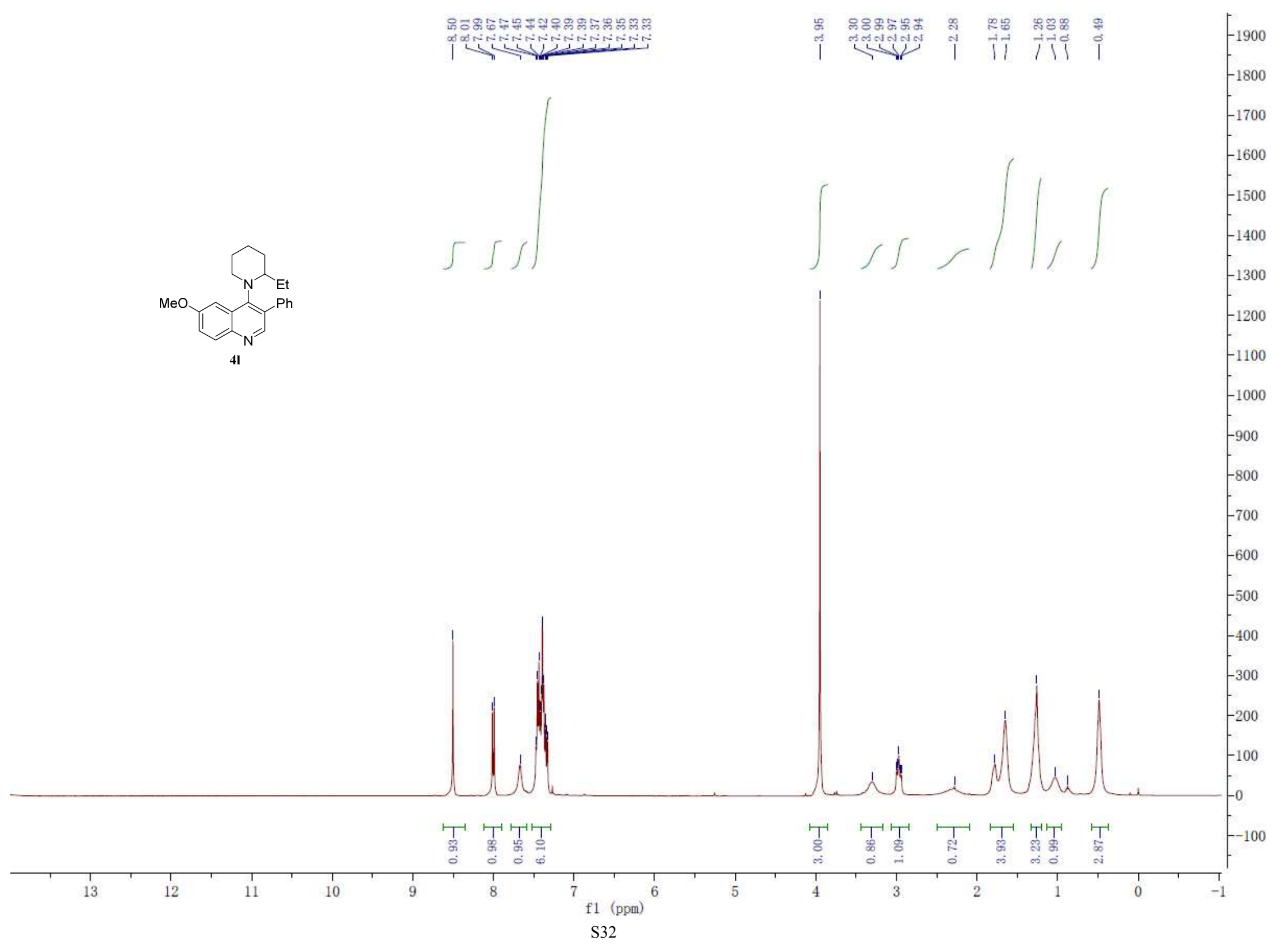




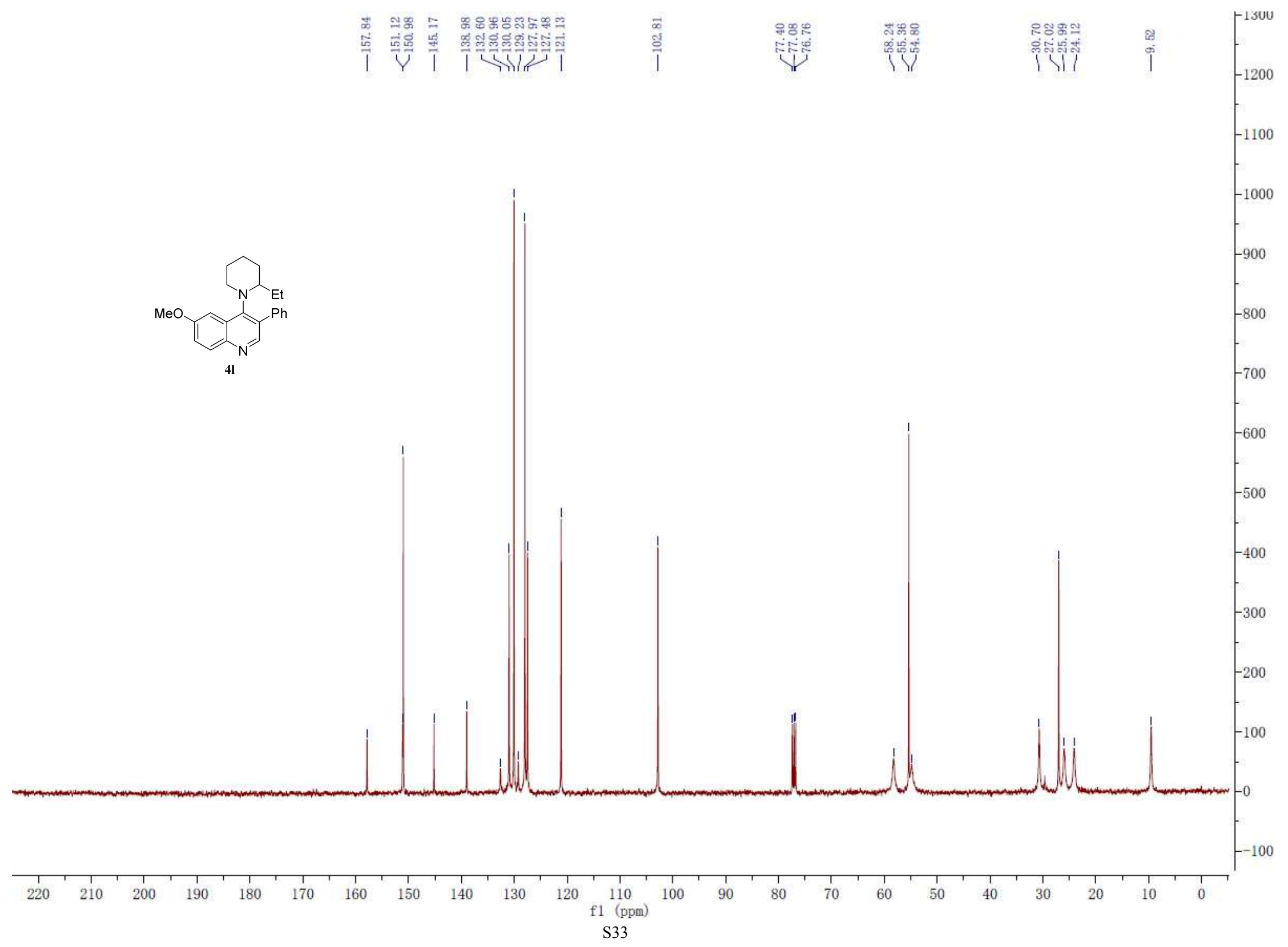




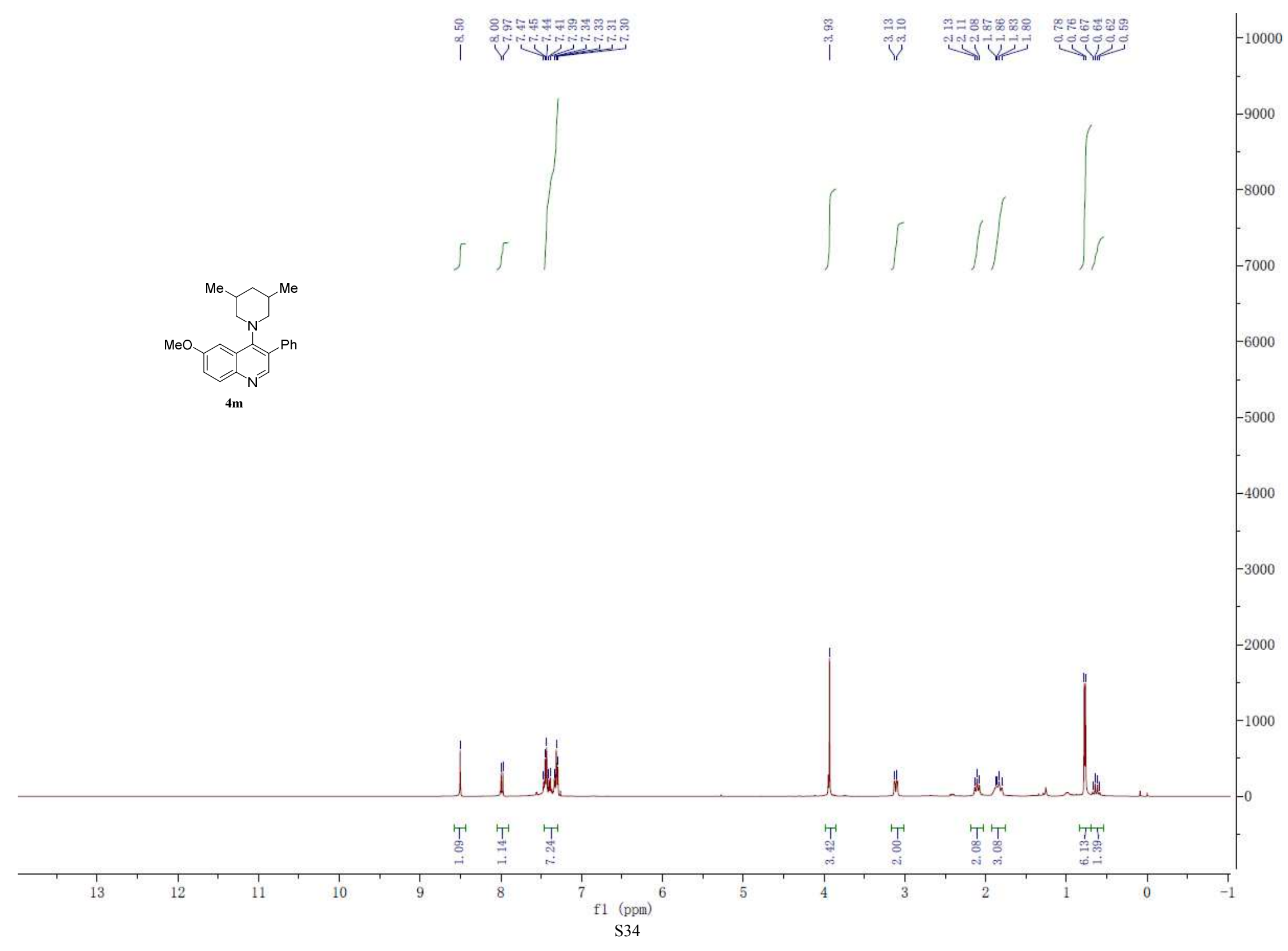




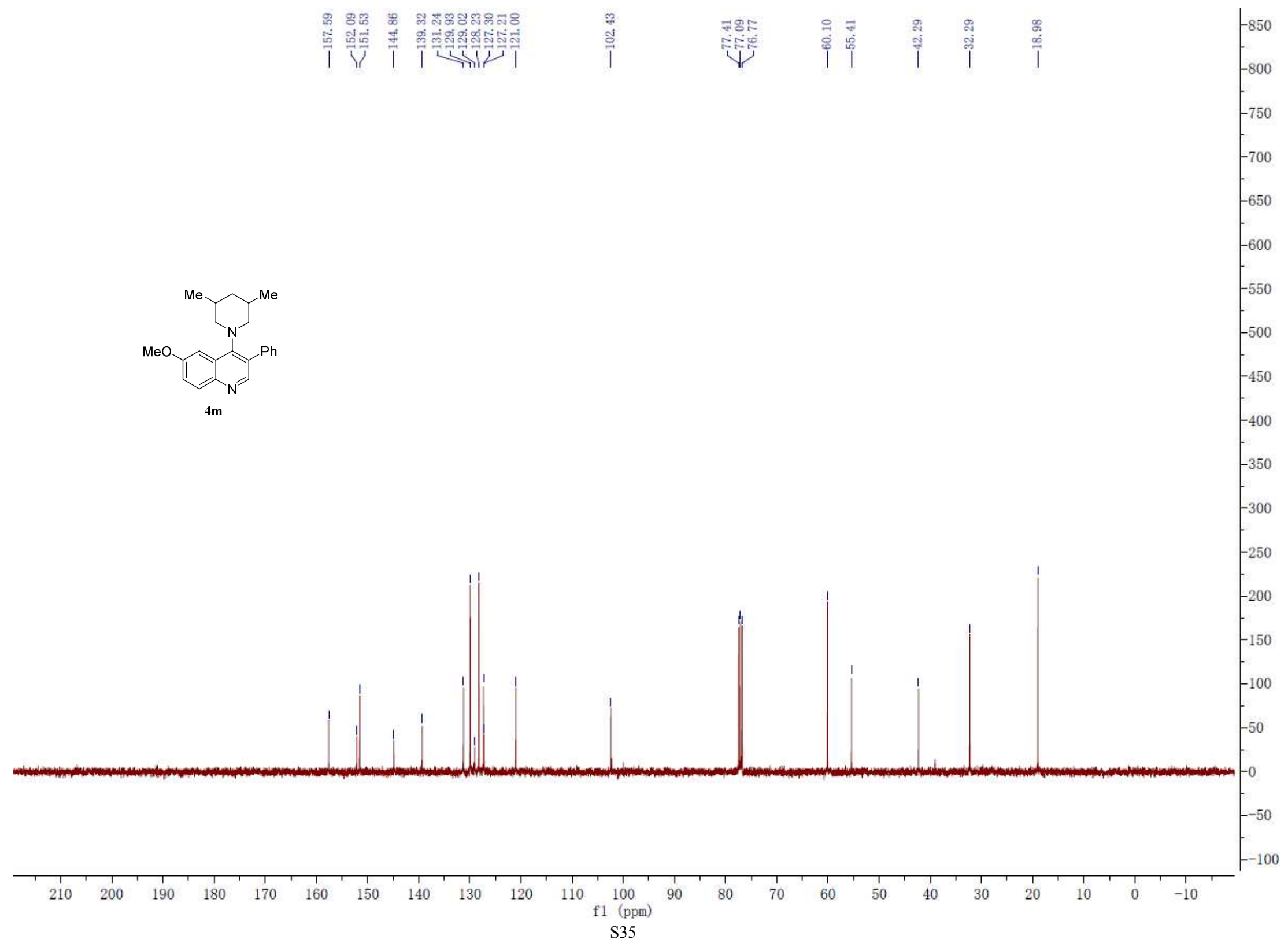




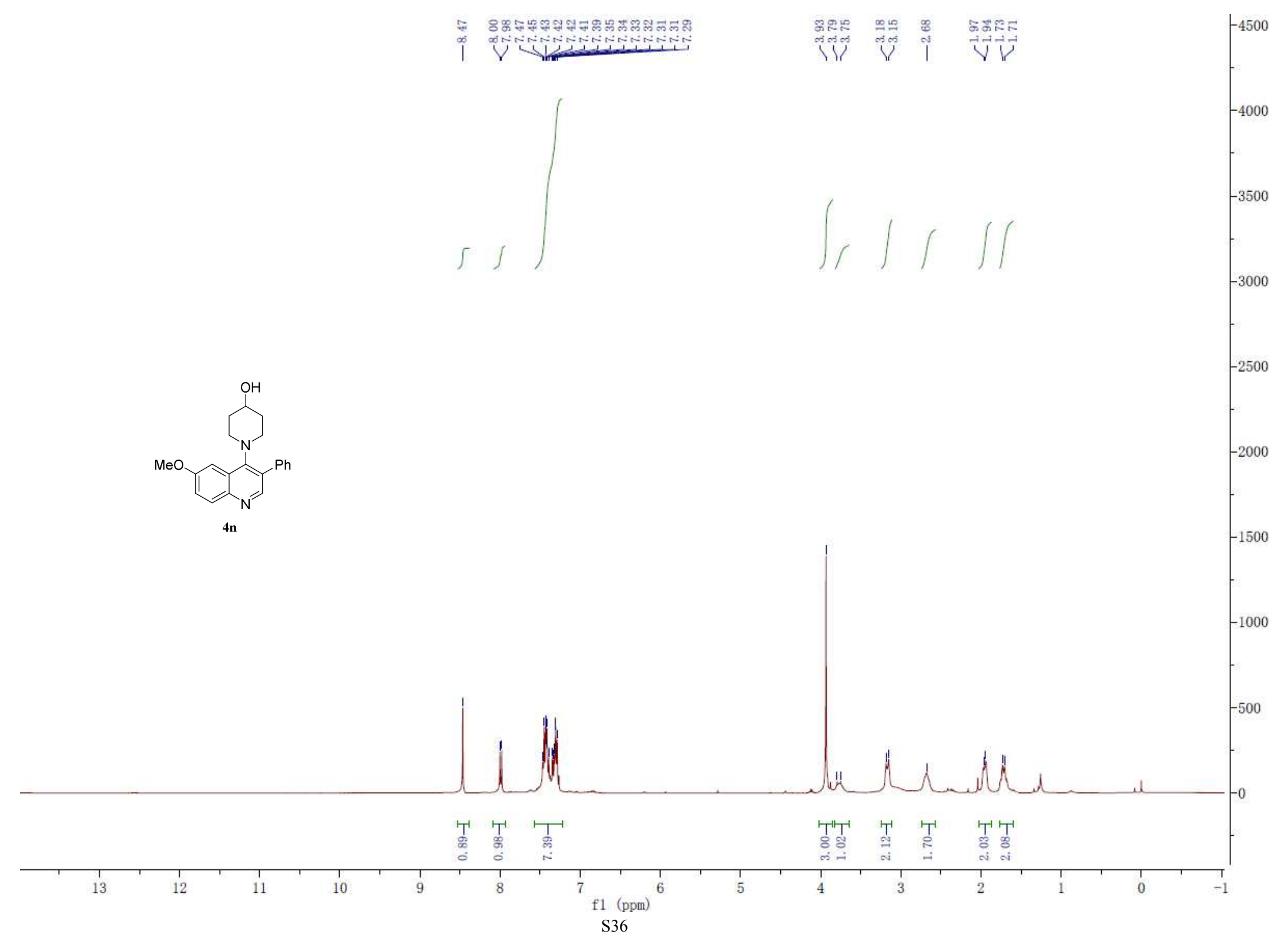




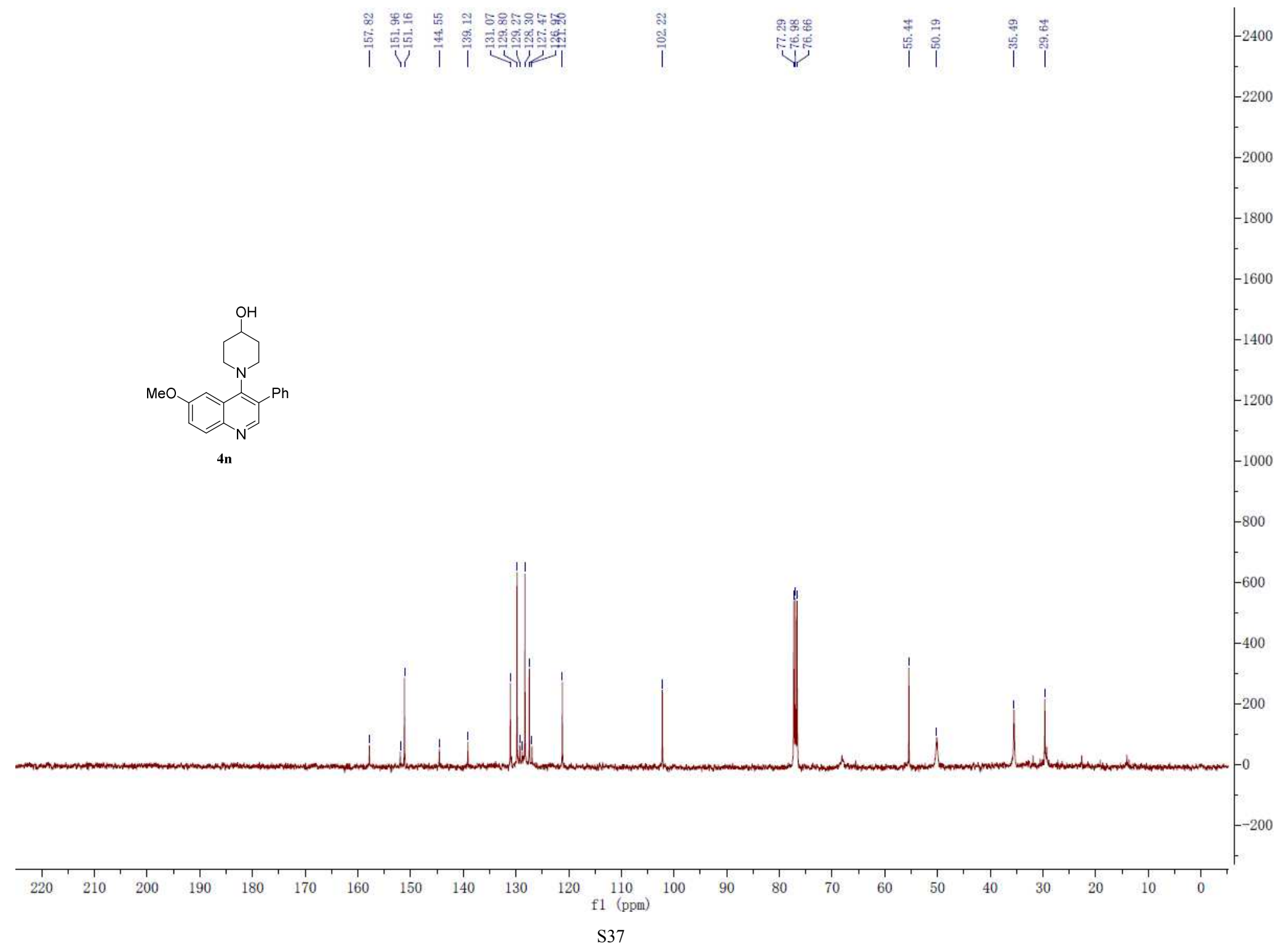




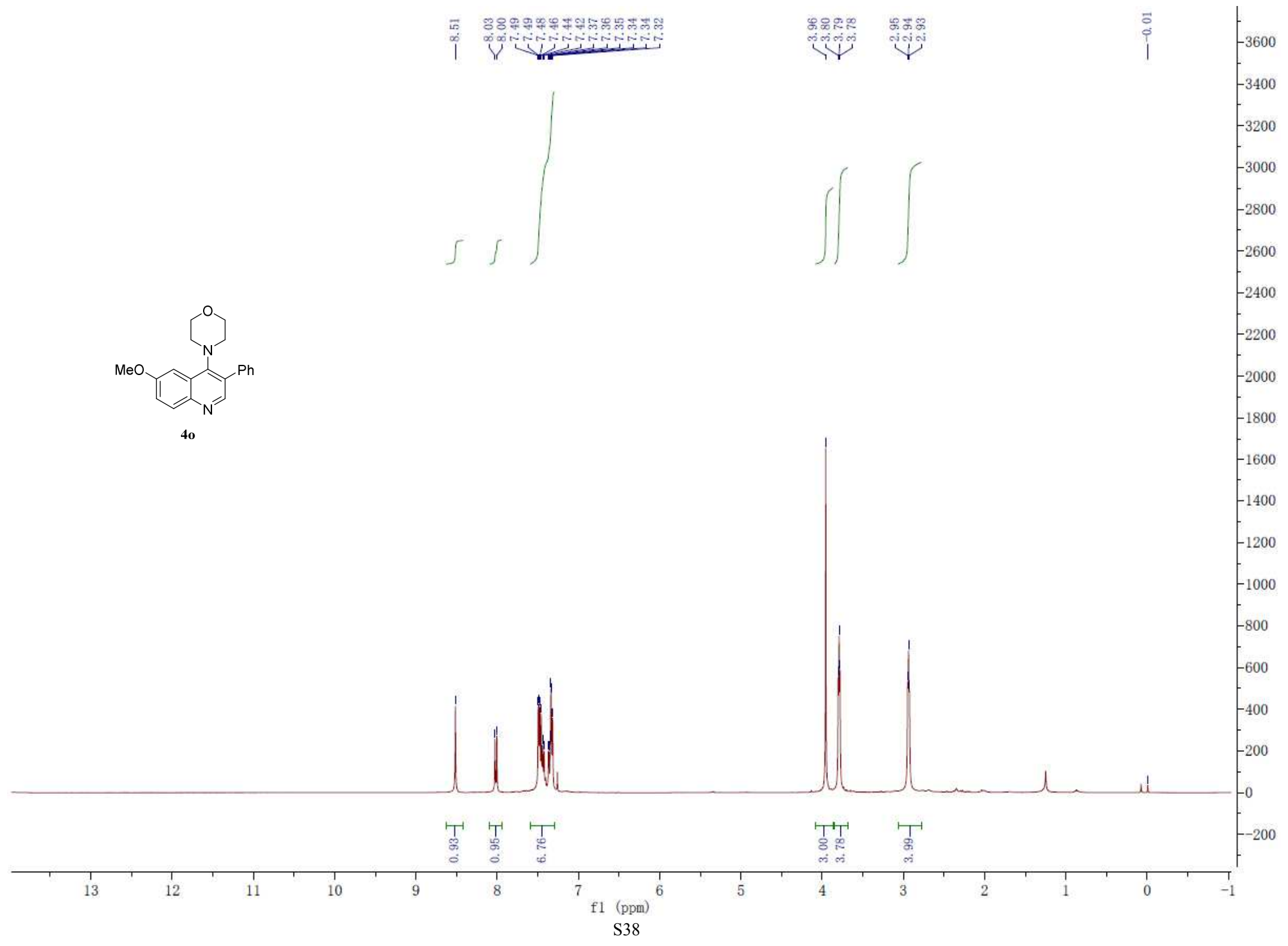




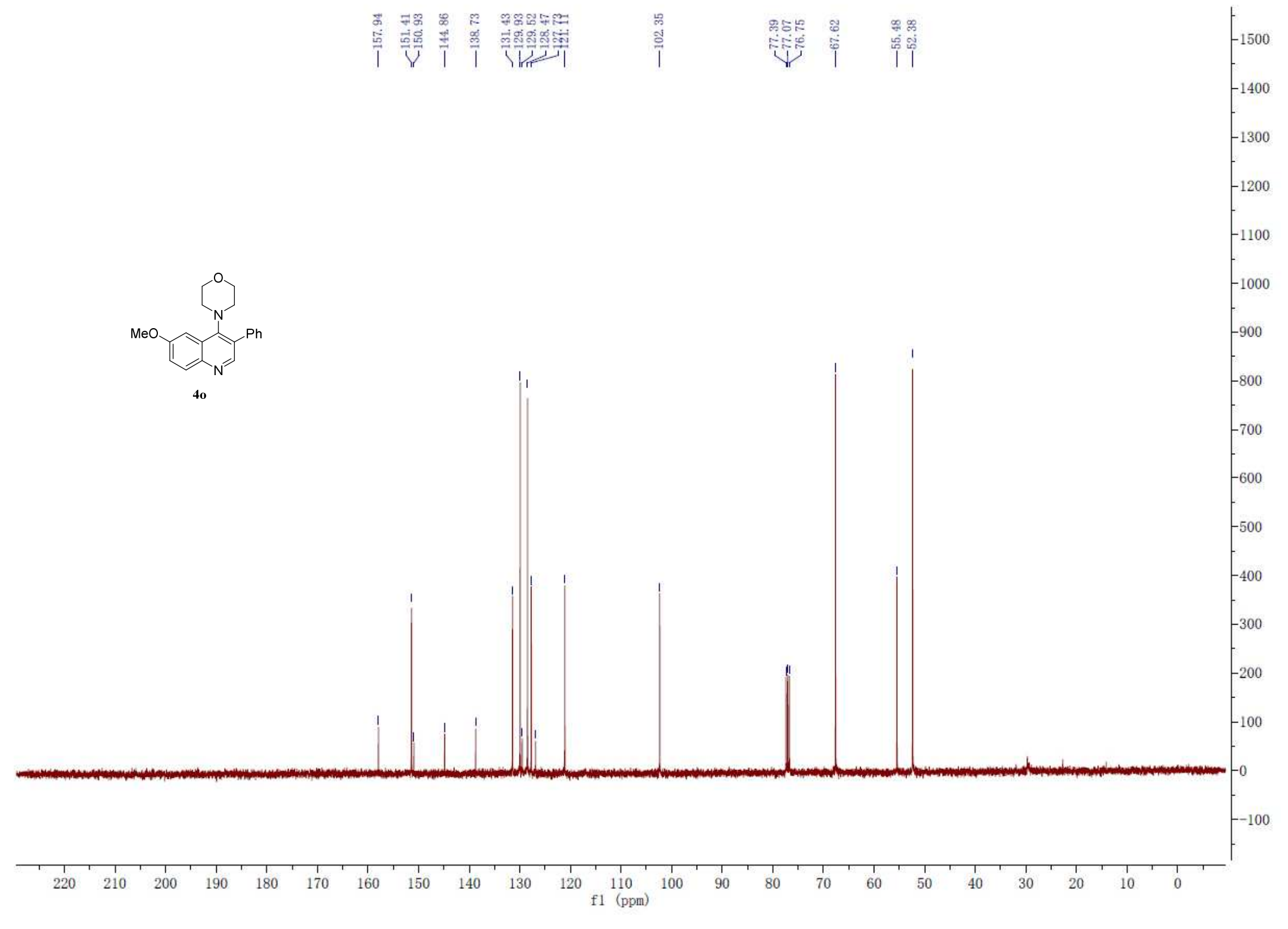




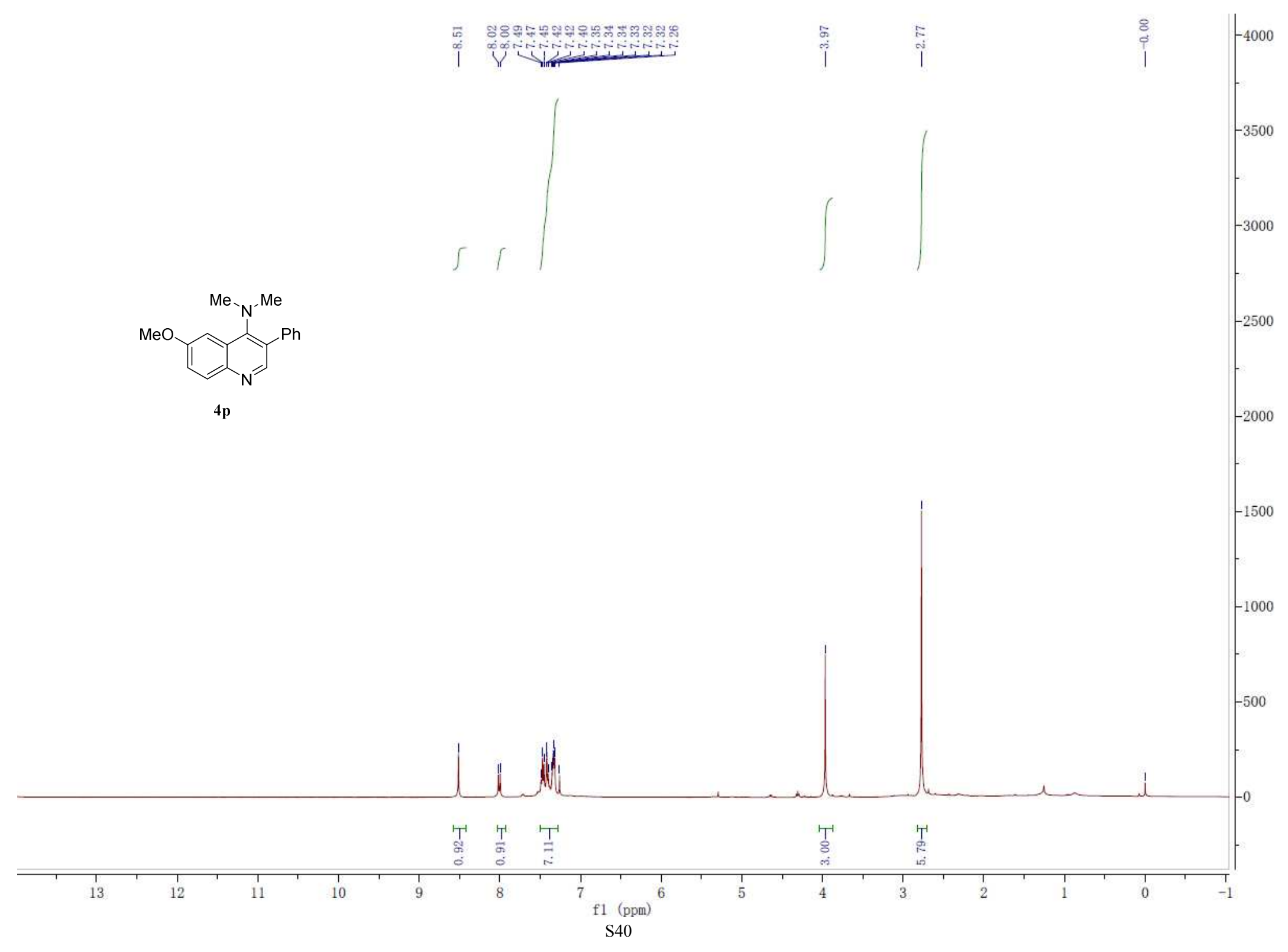




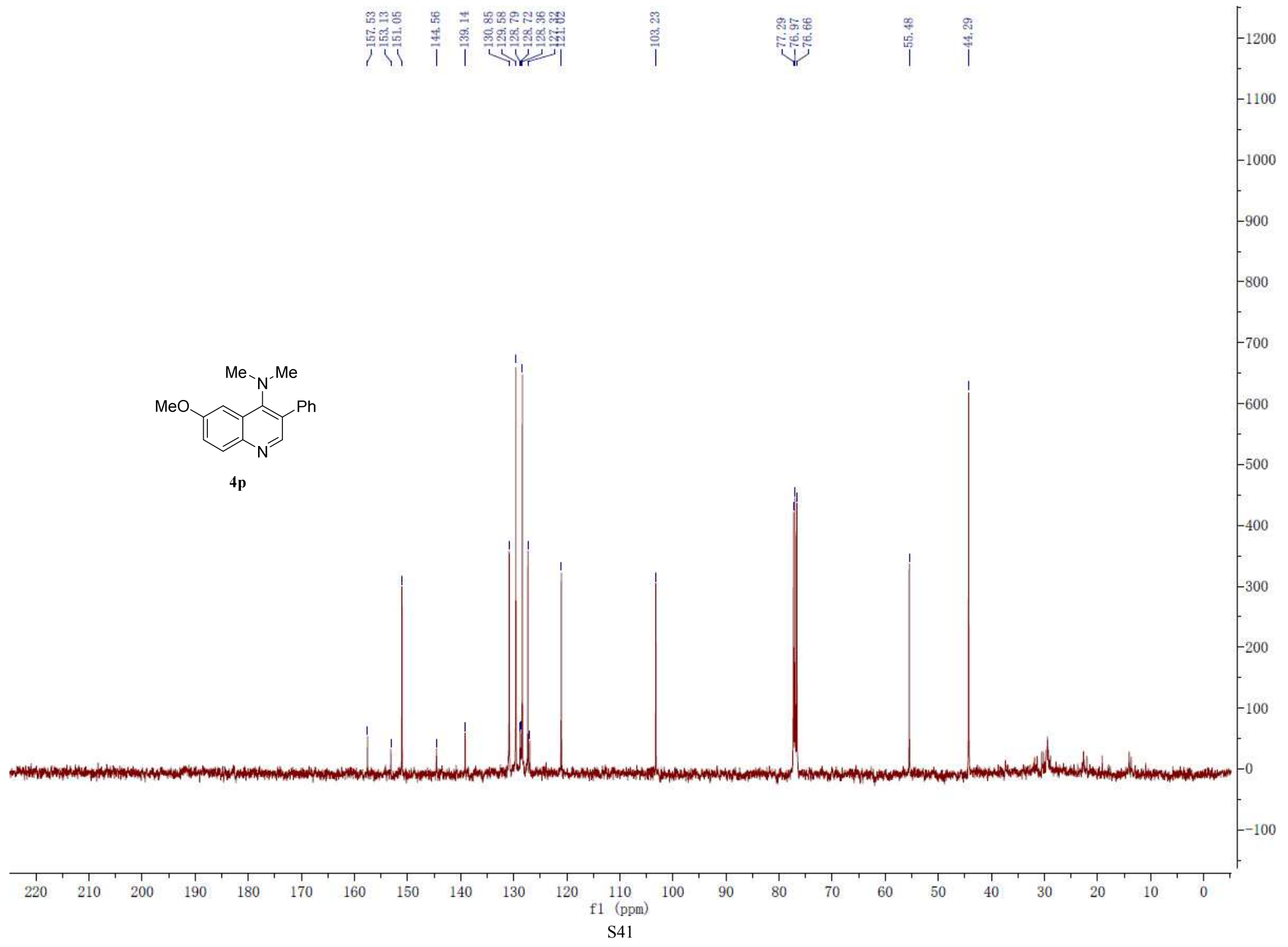




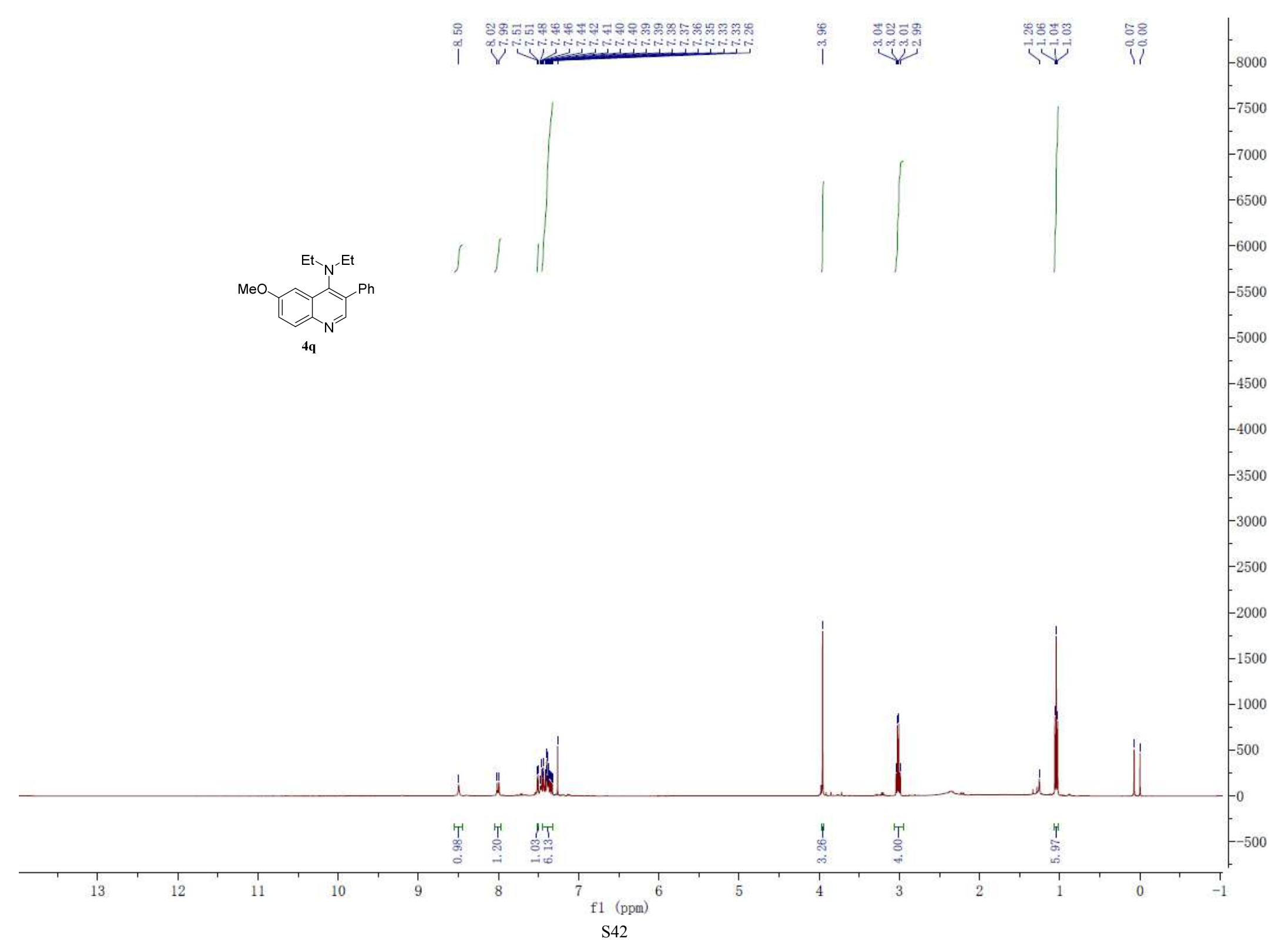




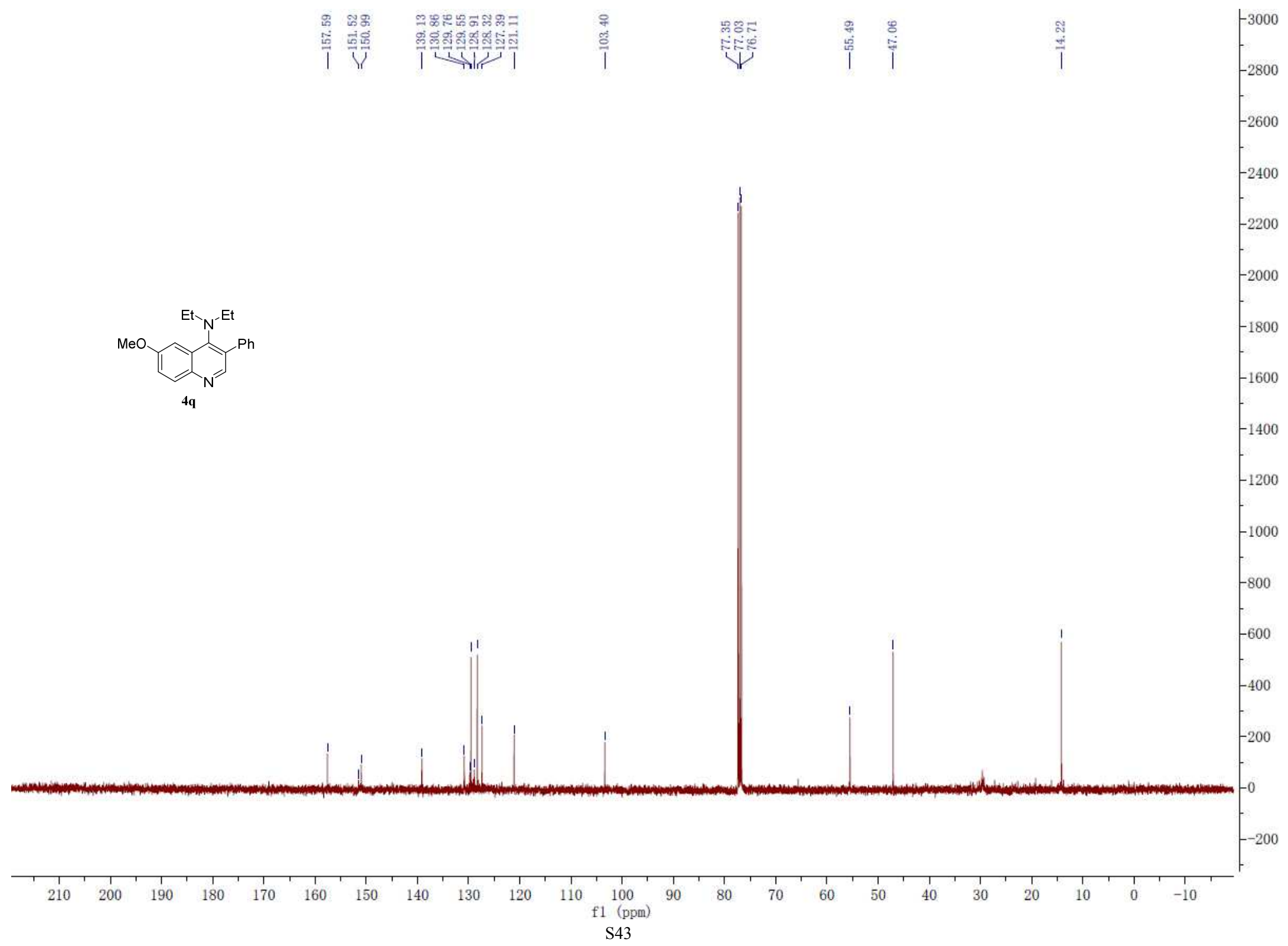




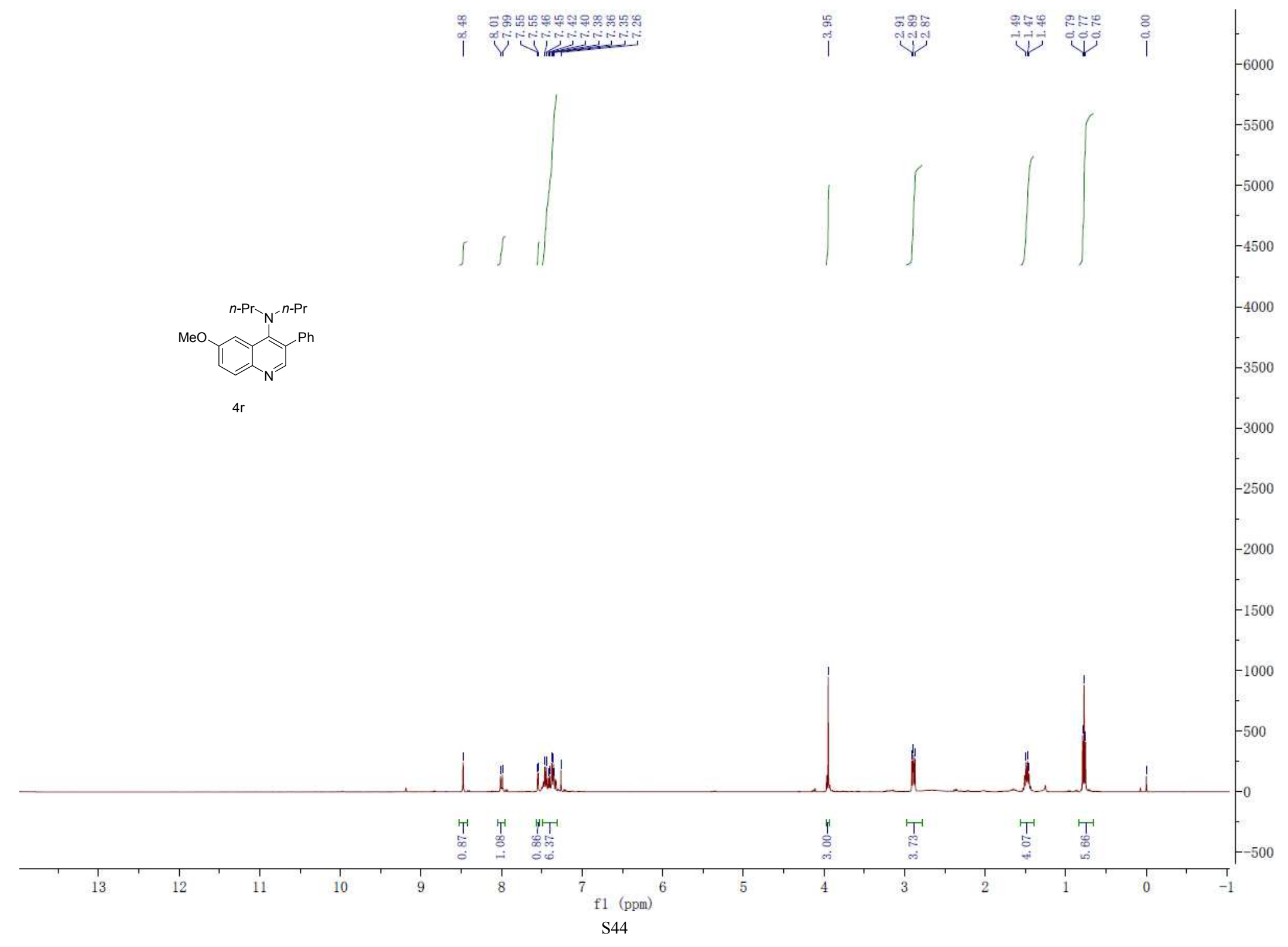




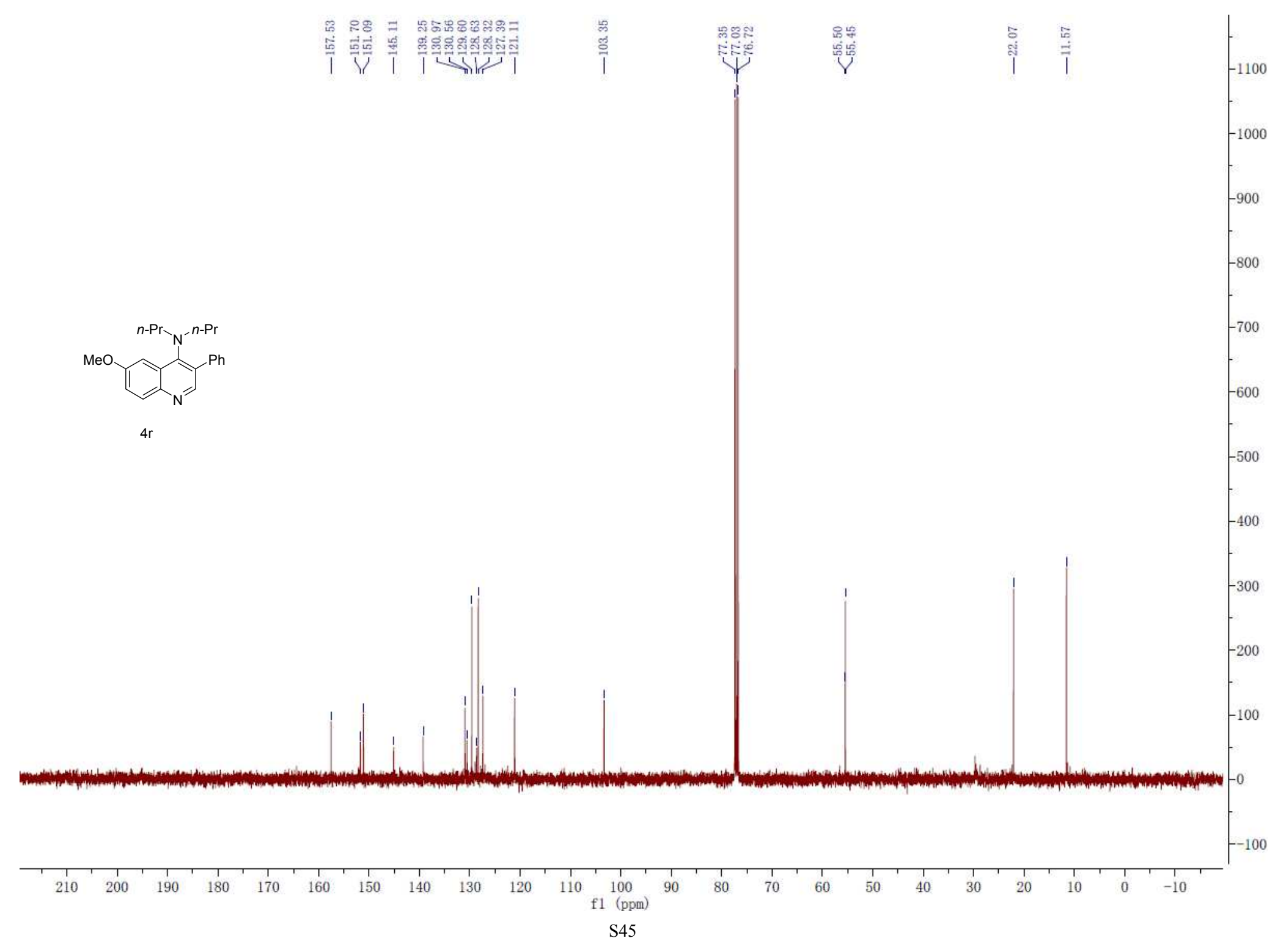




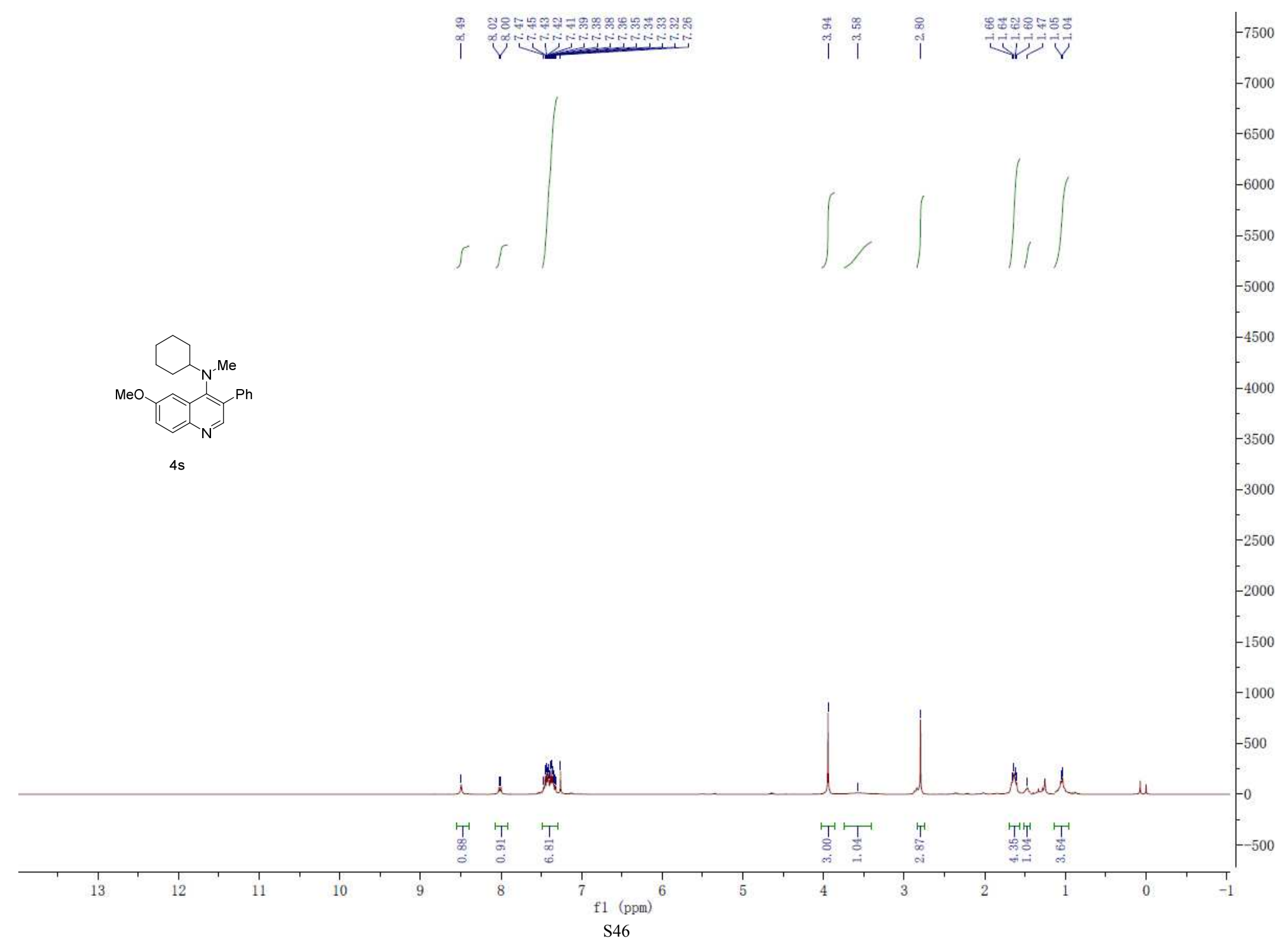




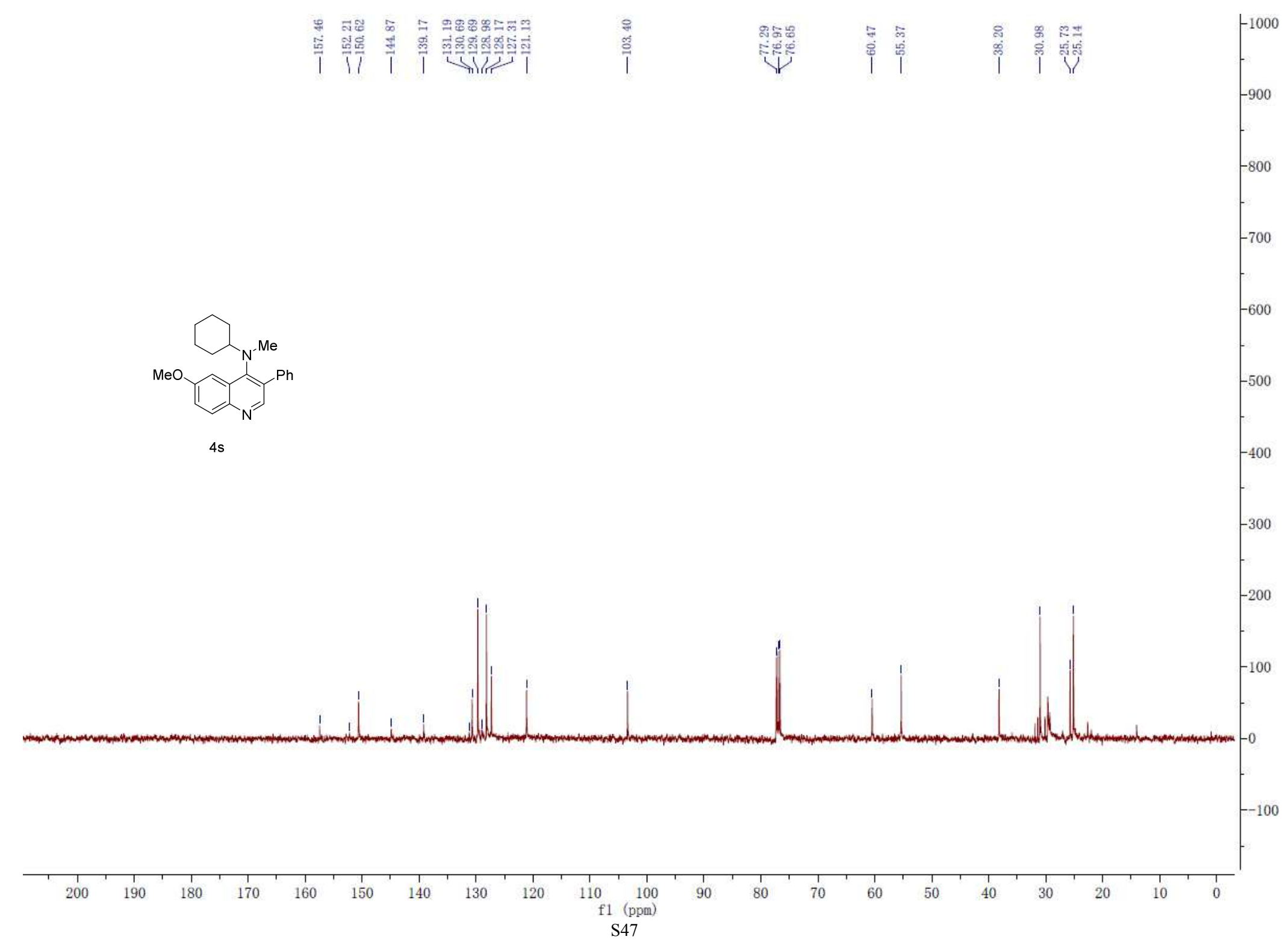




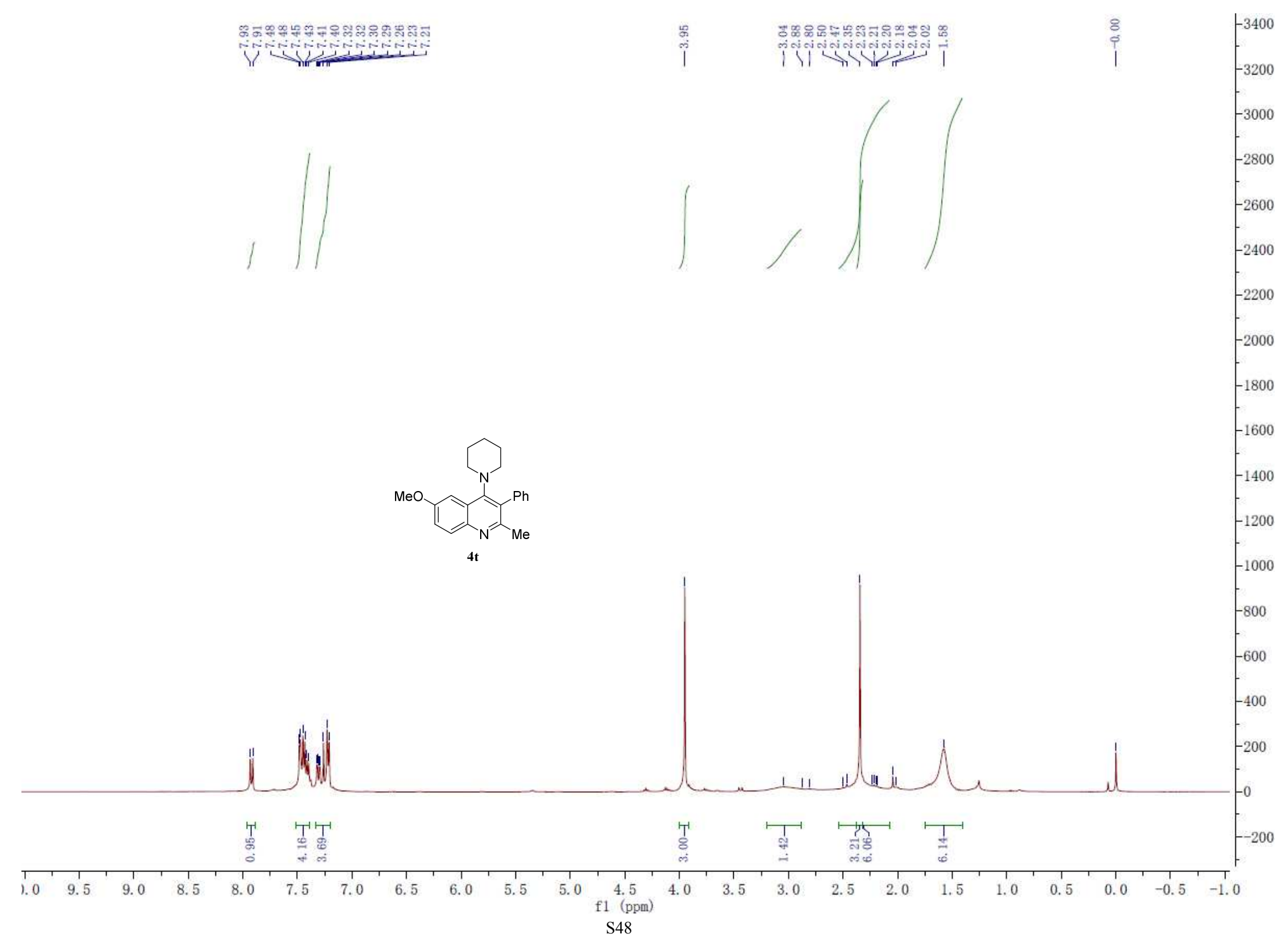









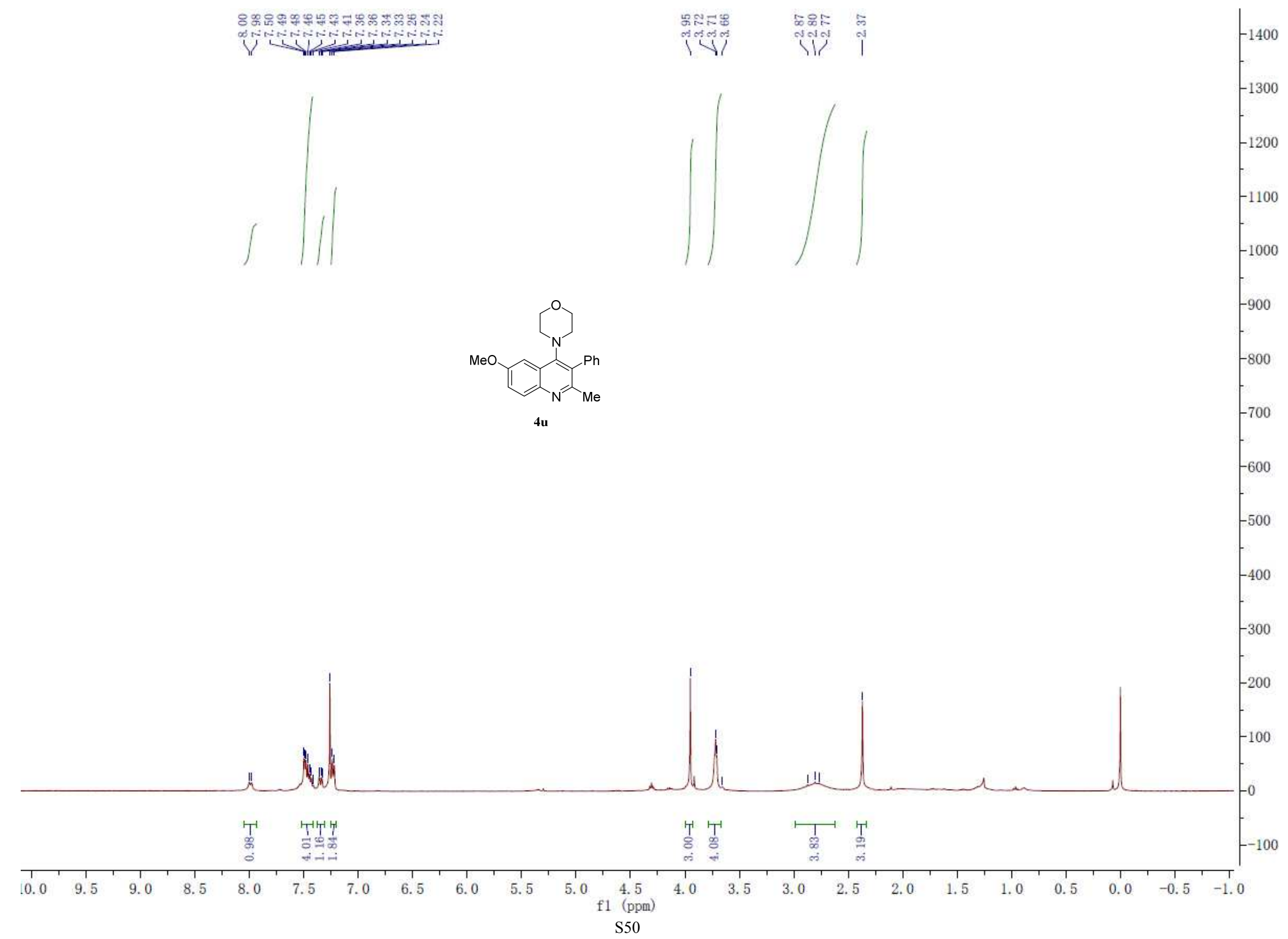




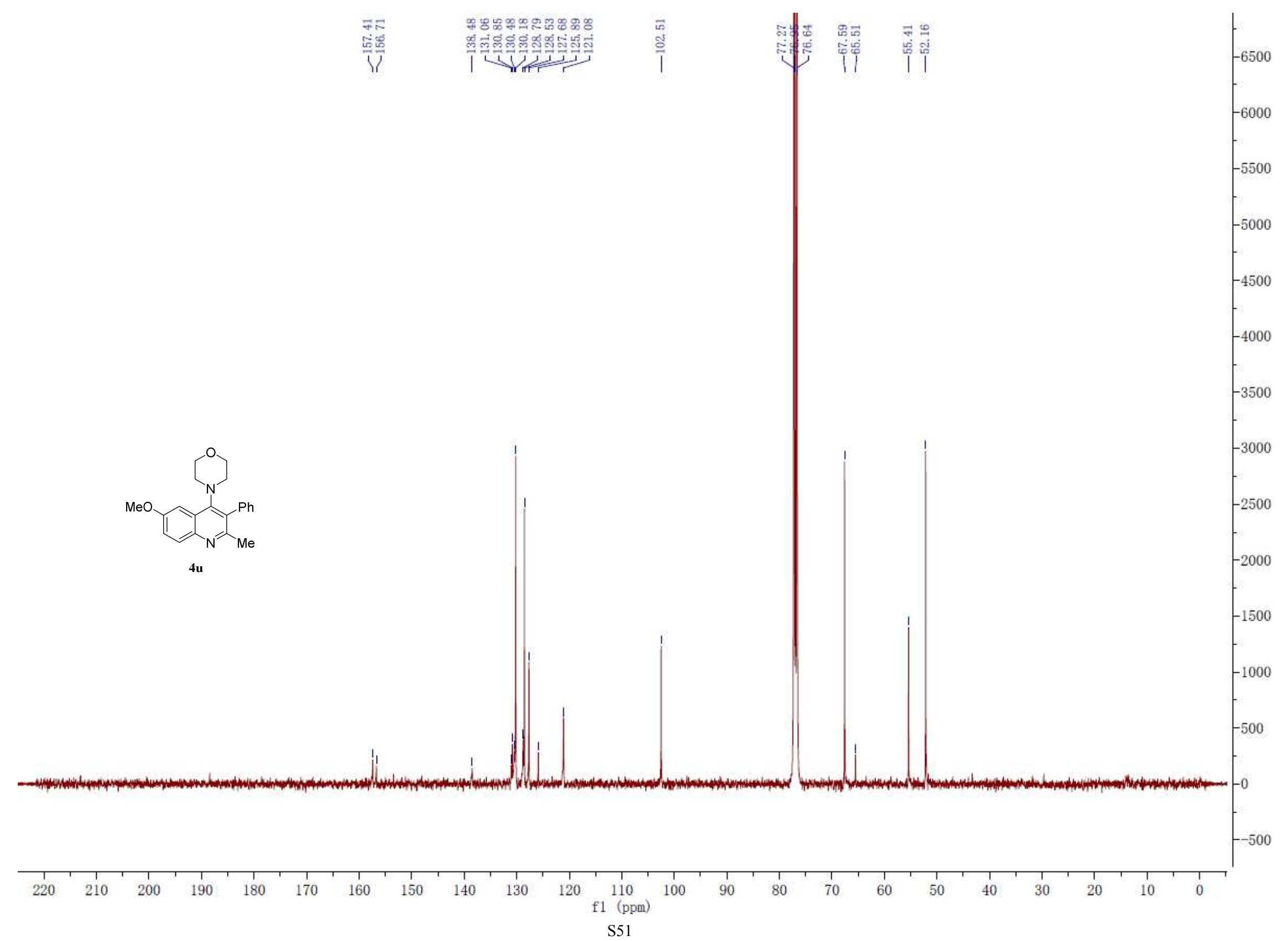




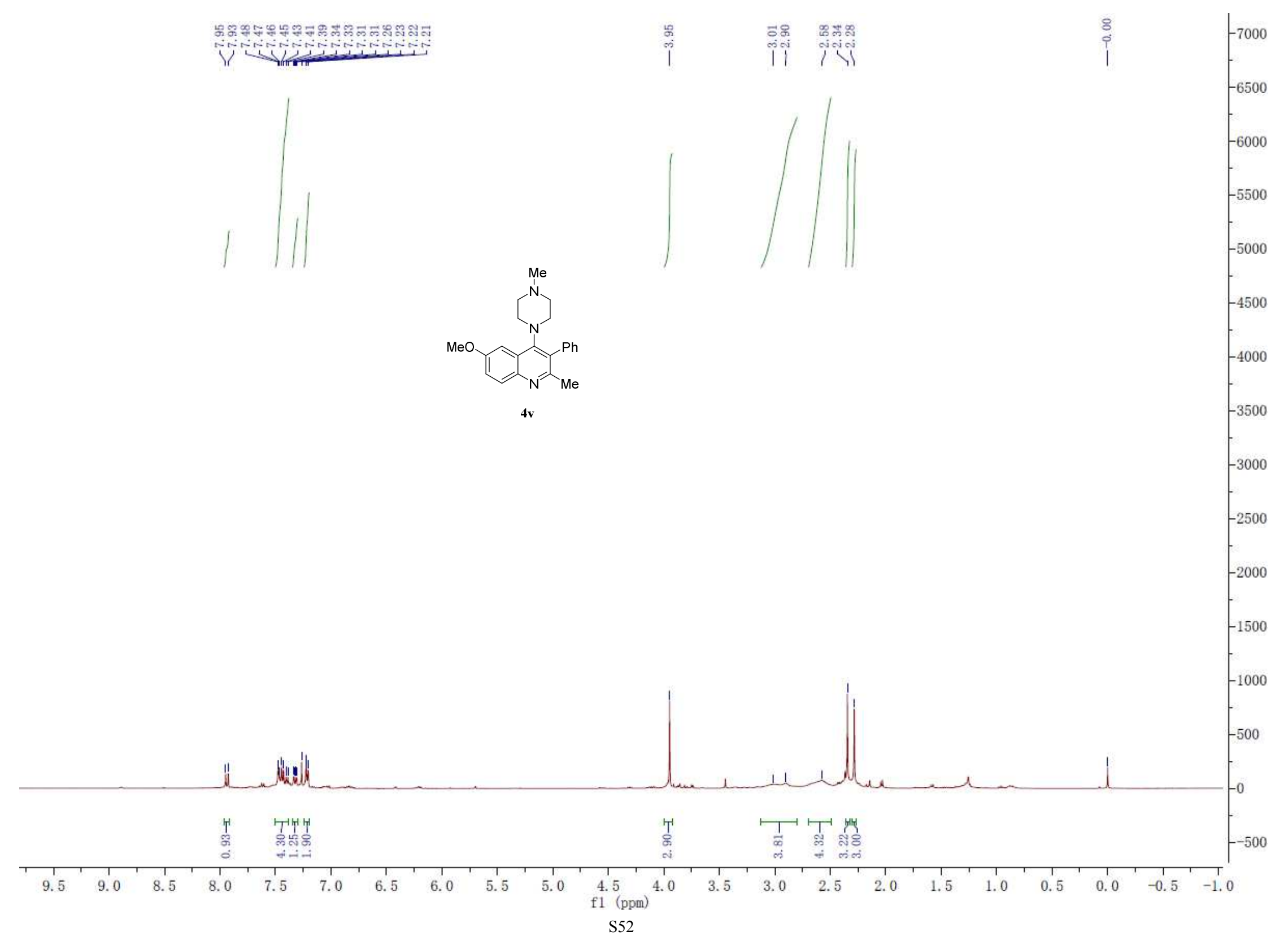




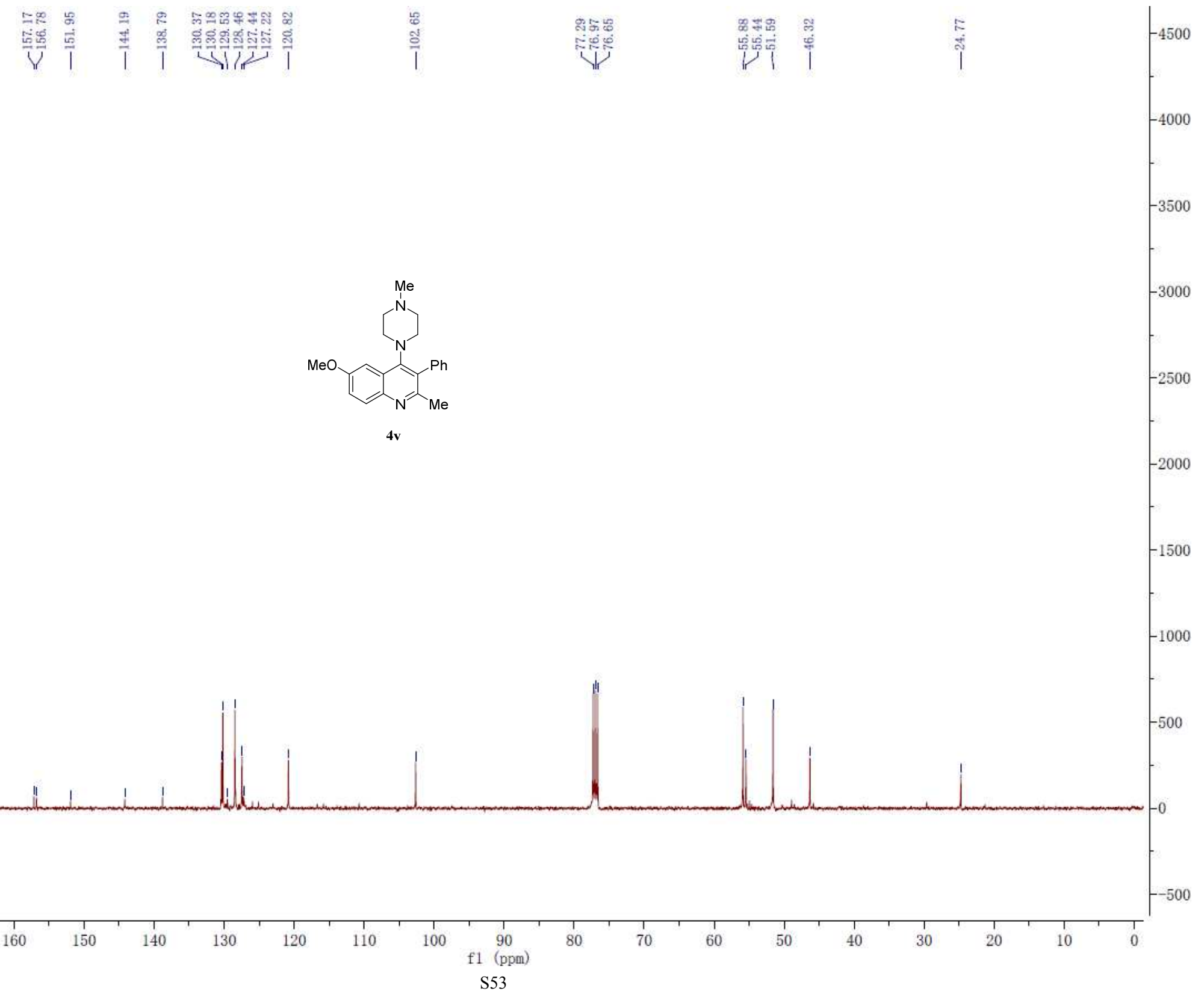




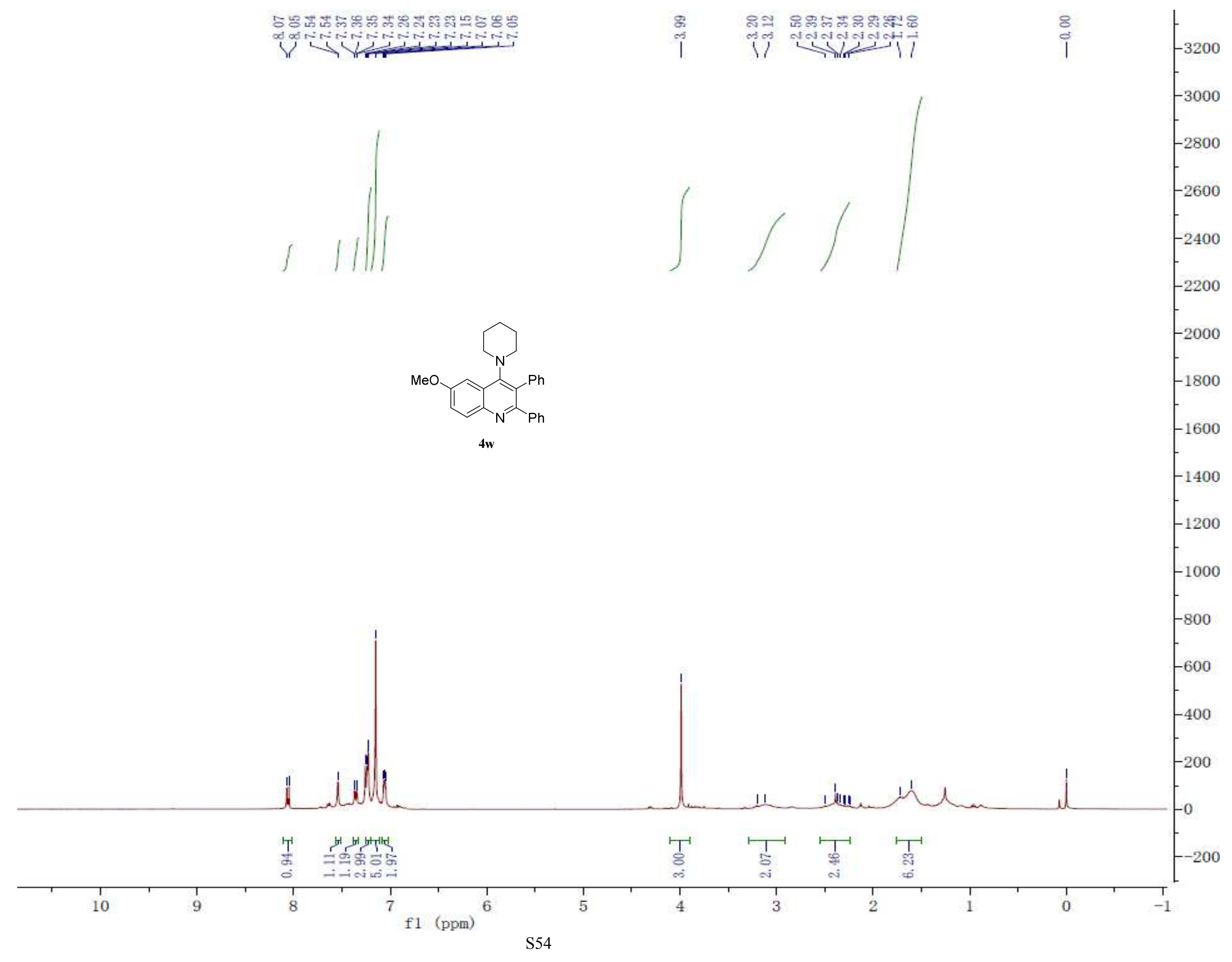




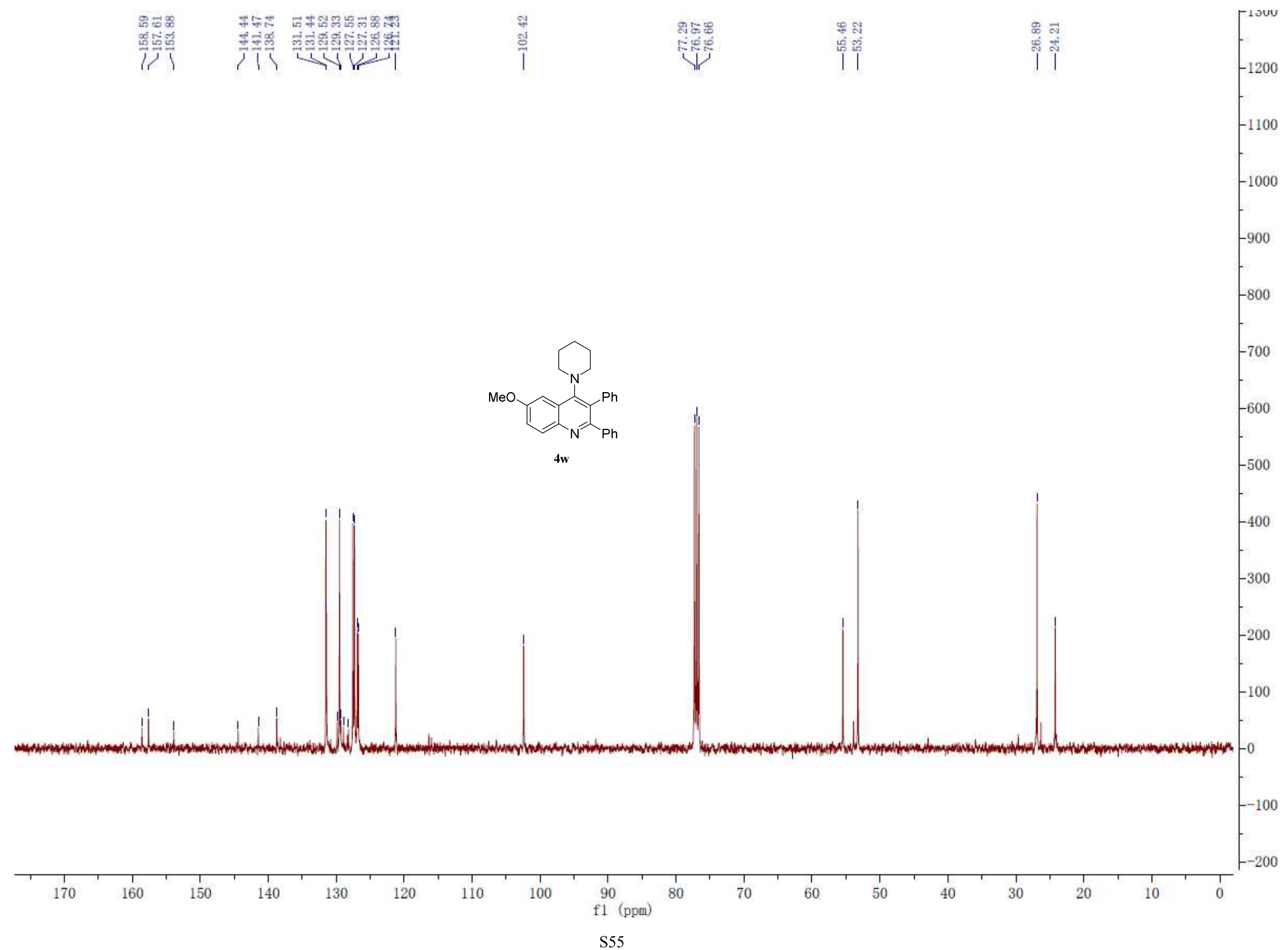




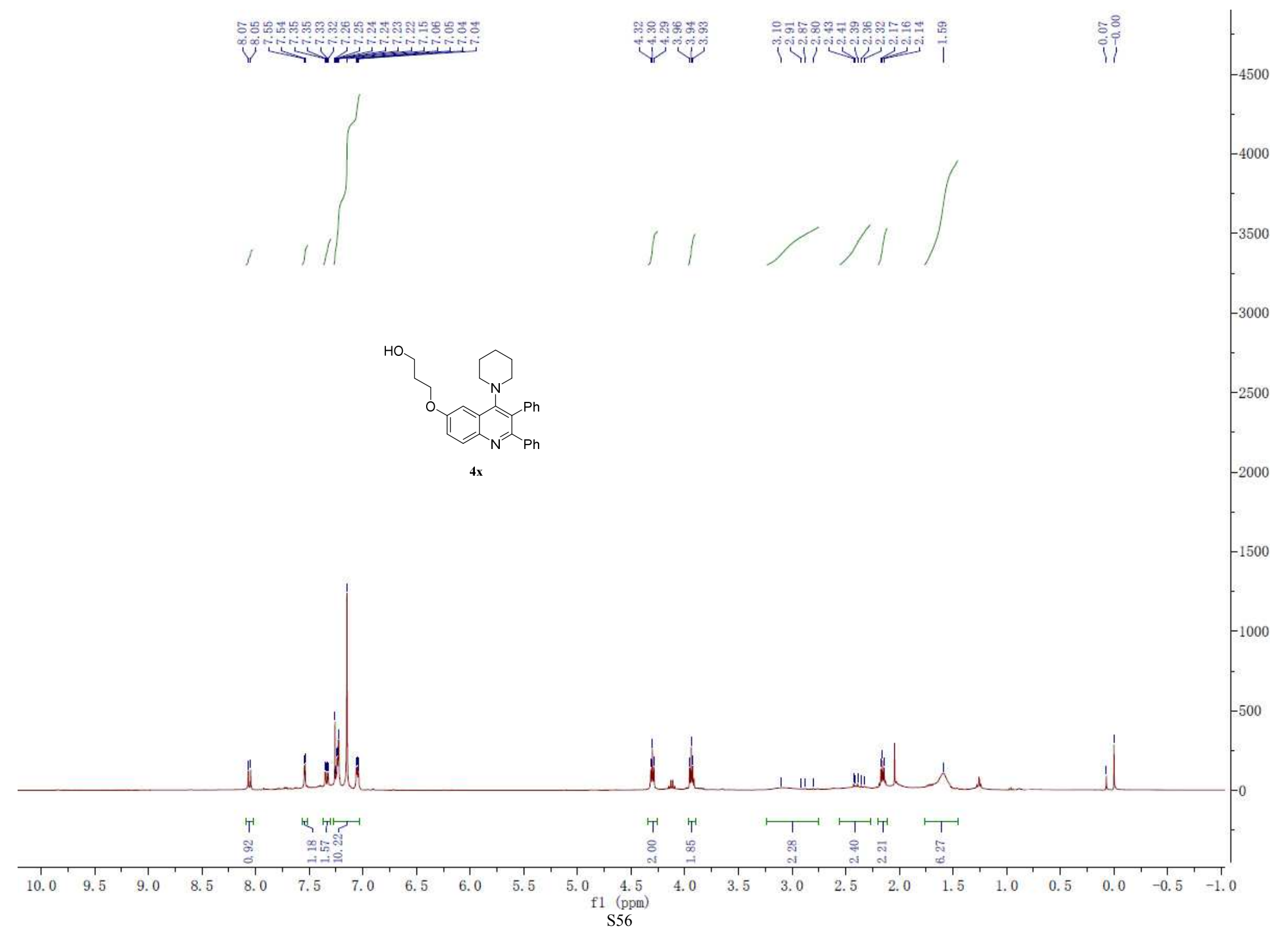




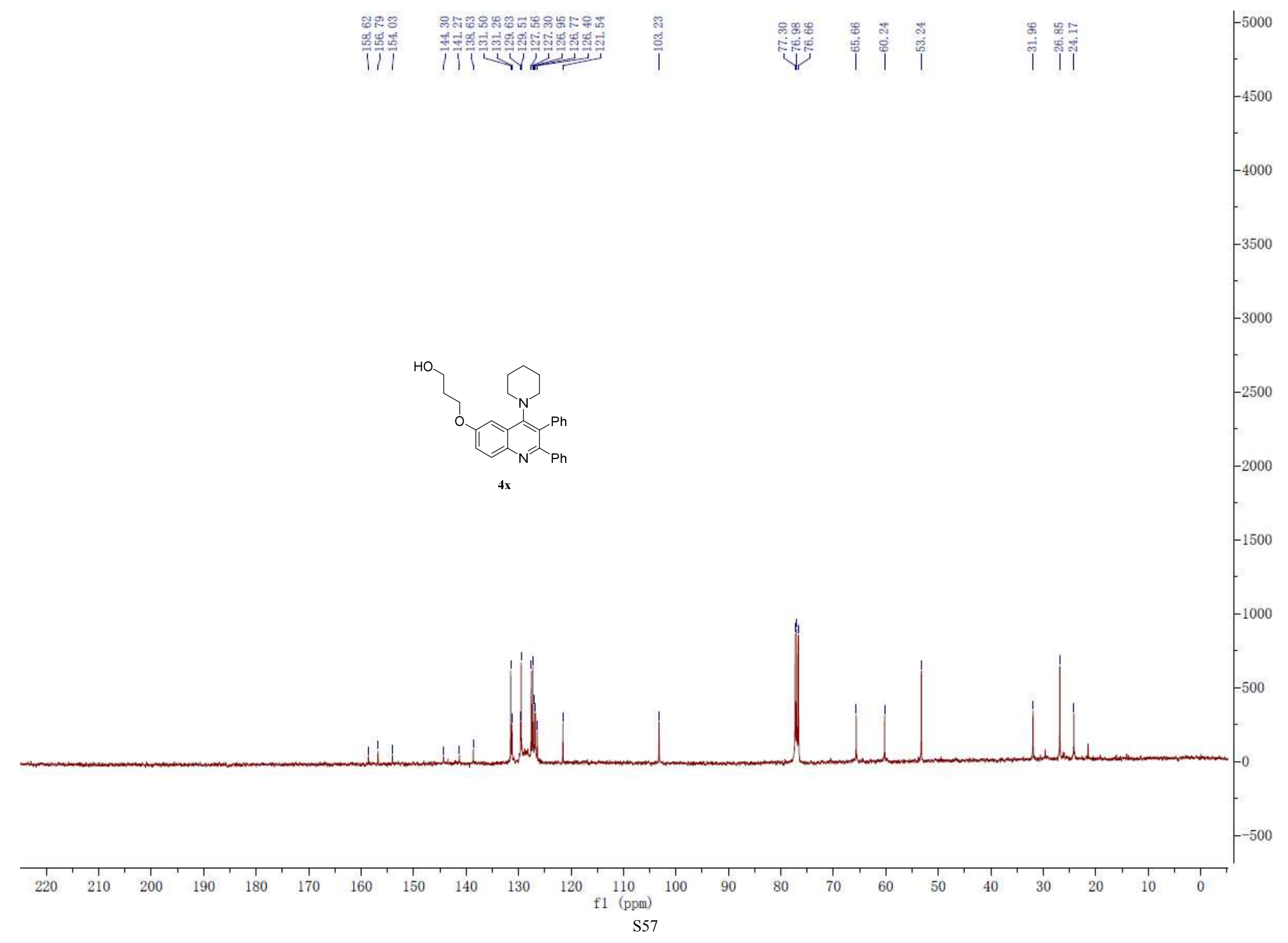




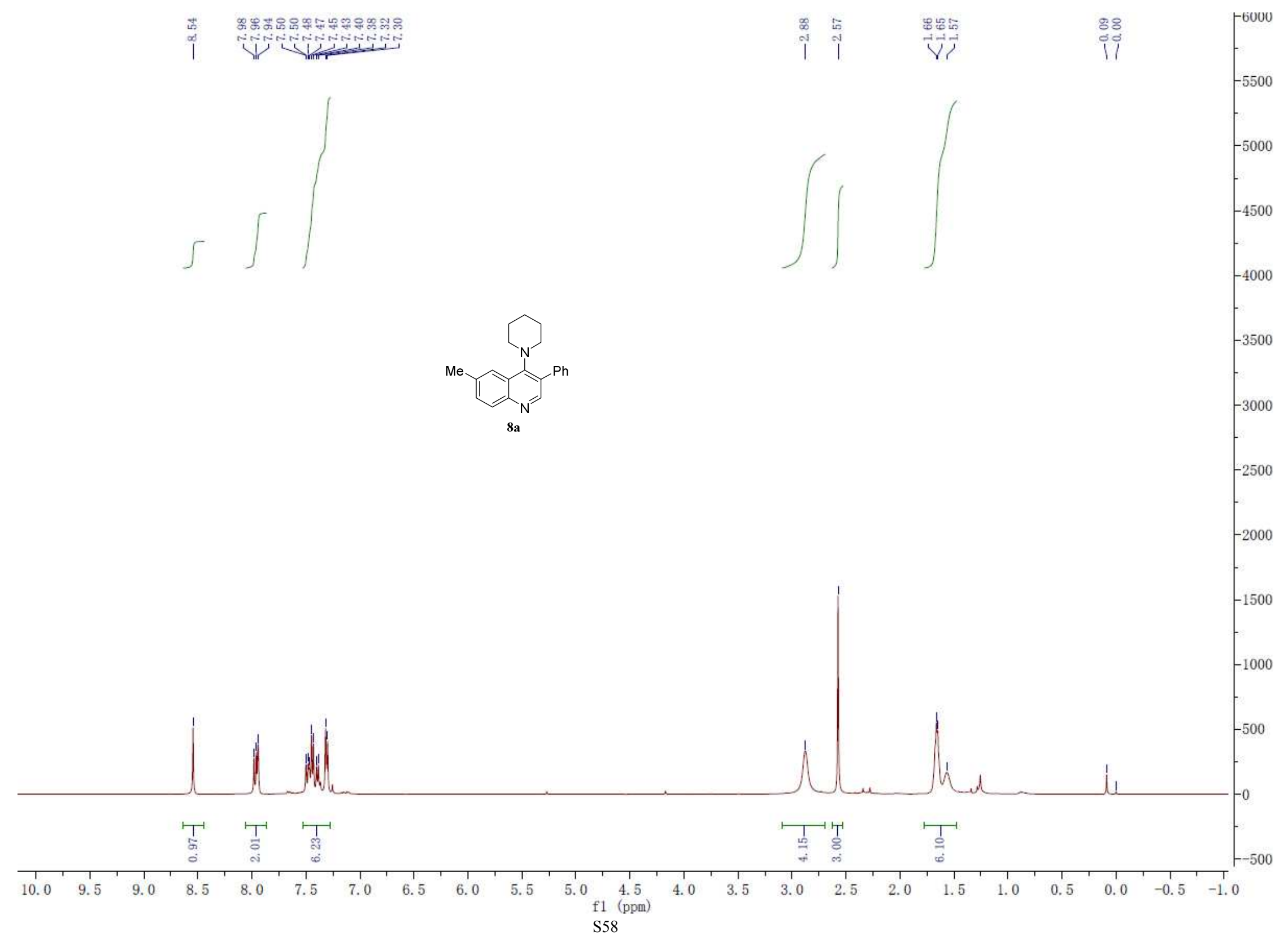




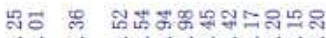

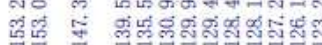

$\checkmark$

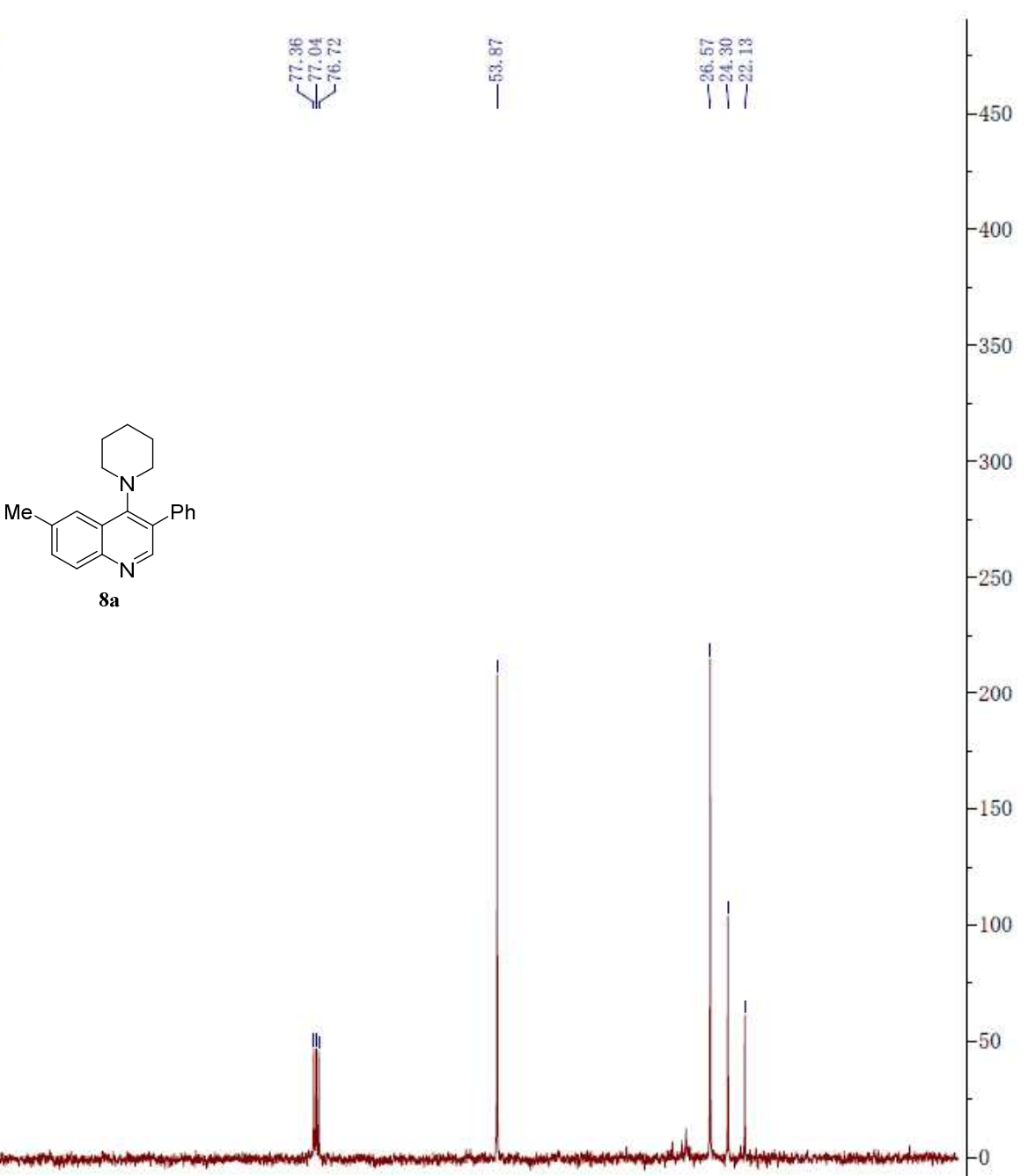

烈

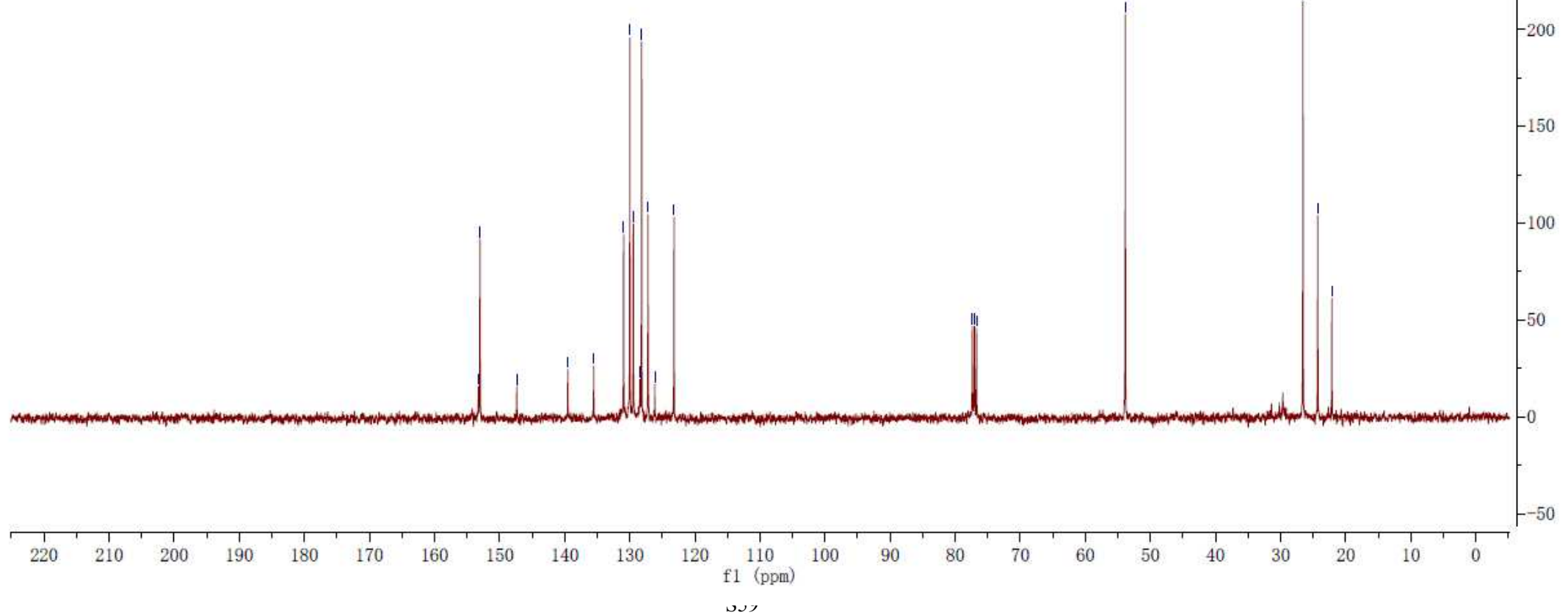




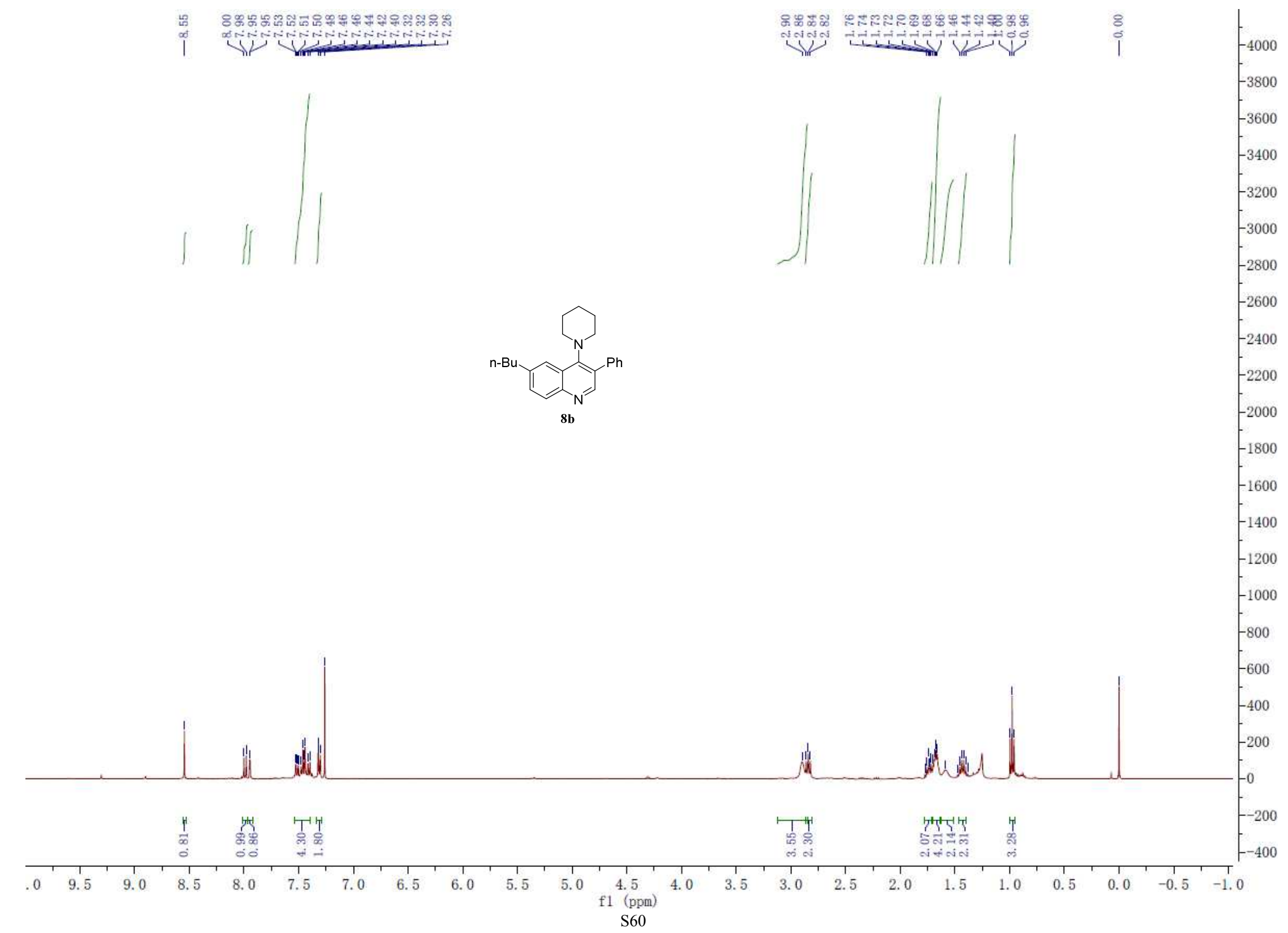




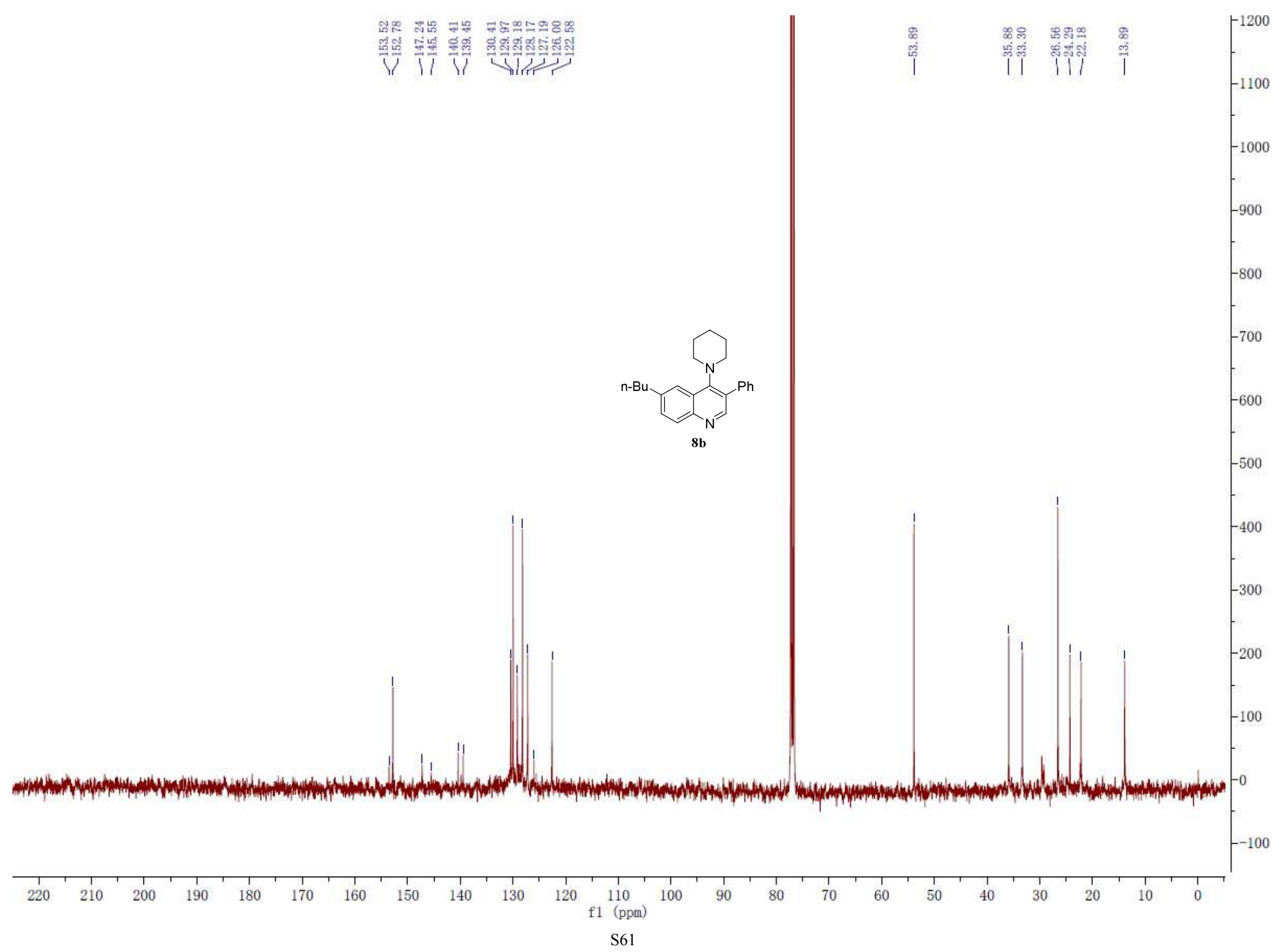




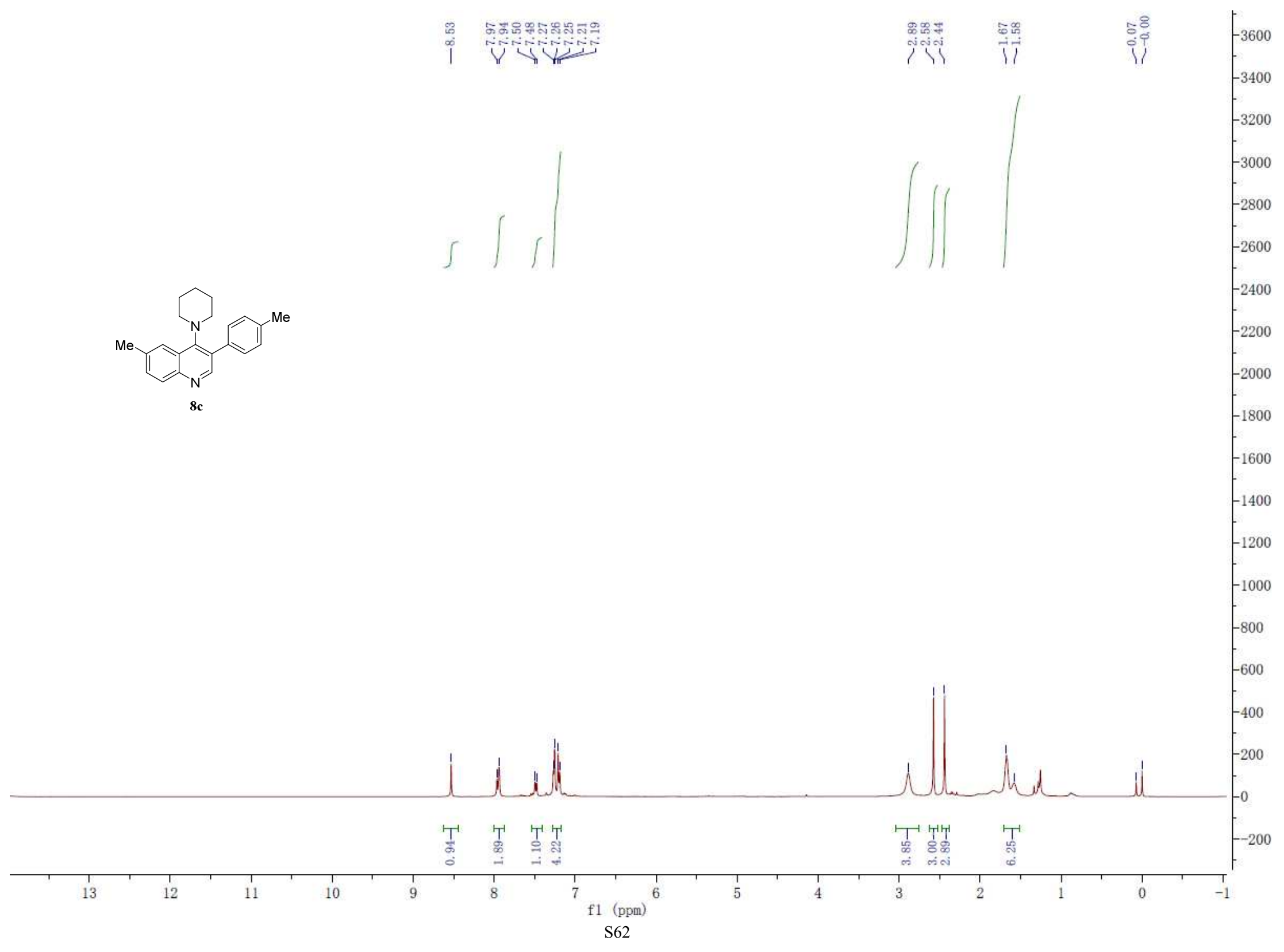




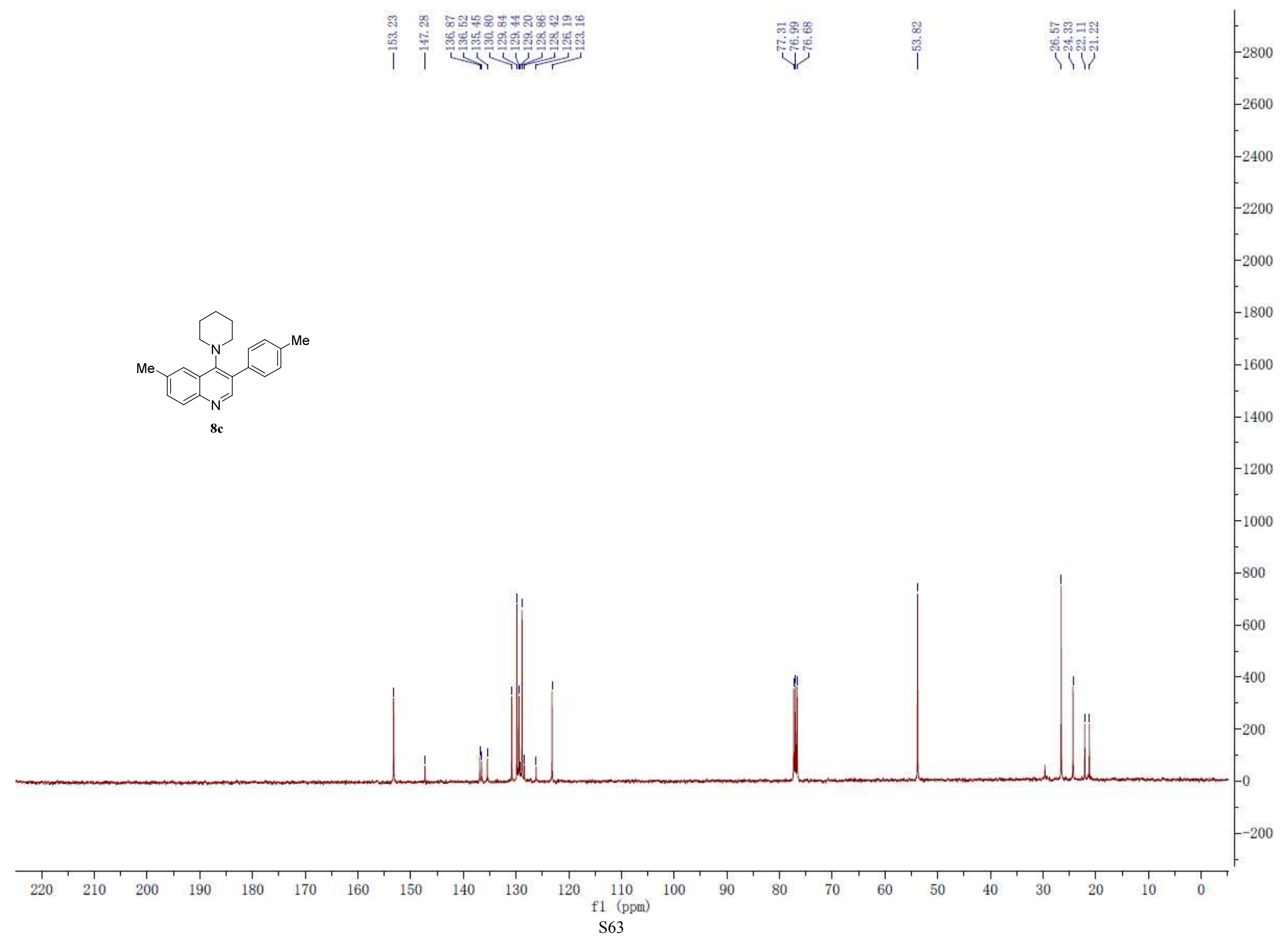




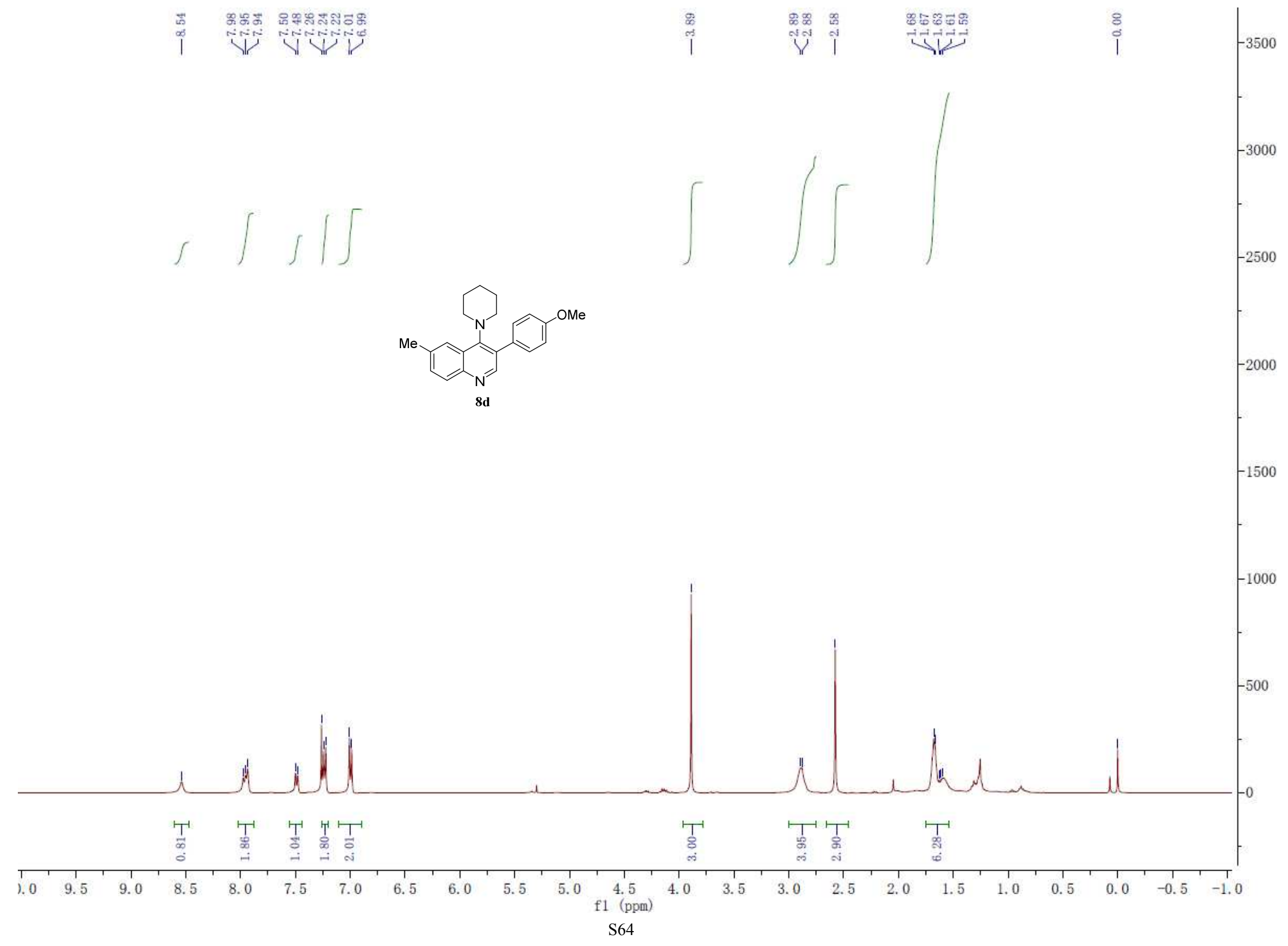




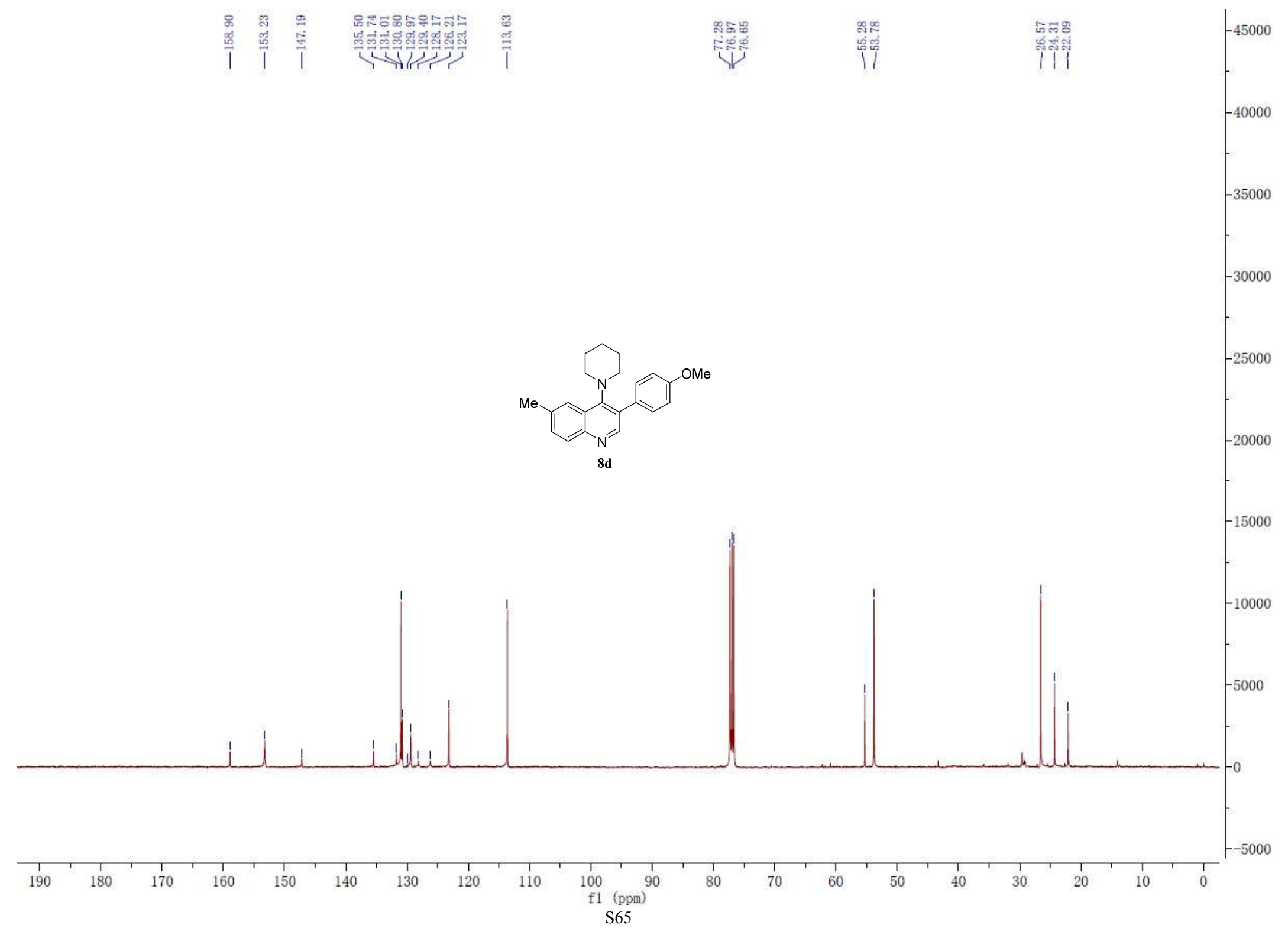




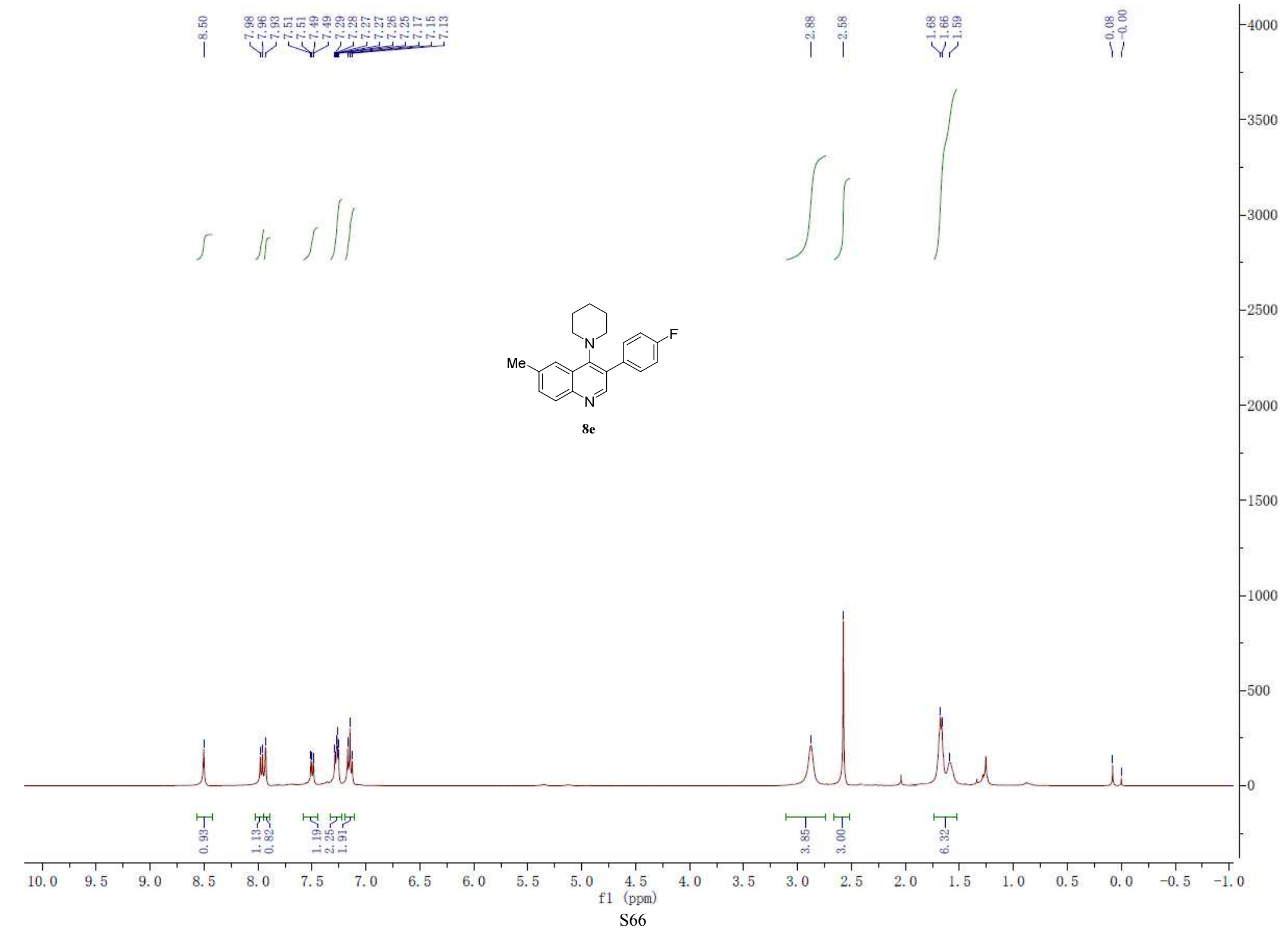




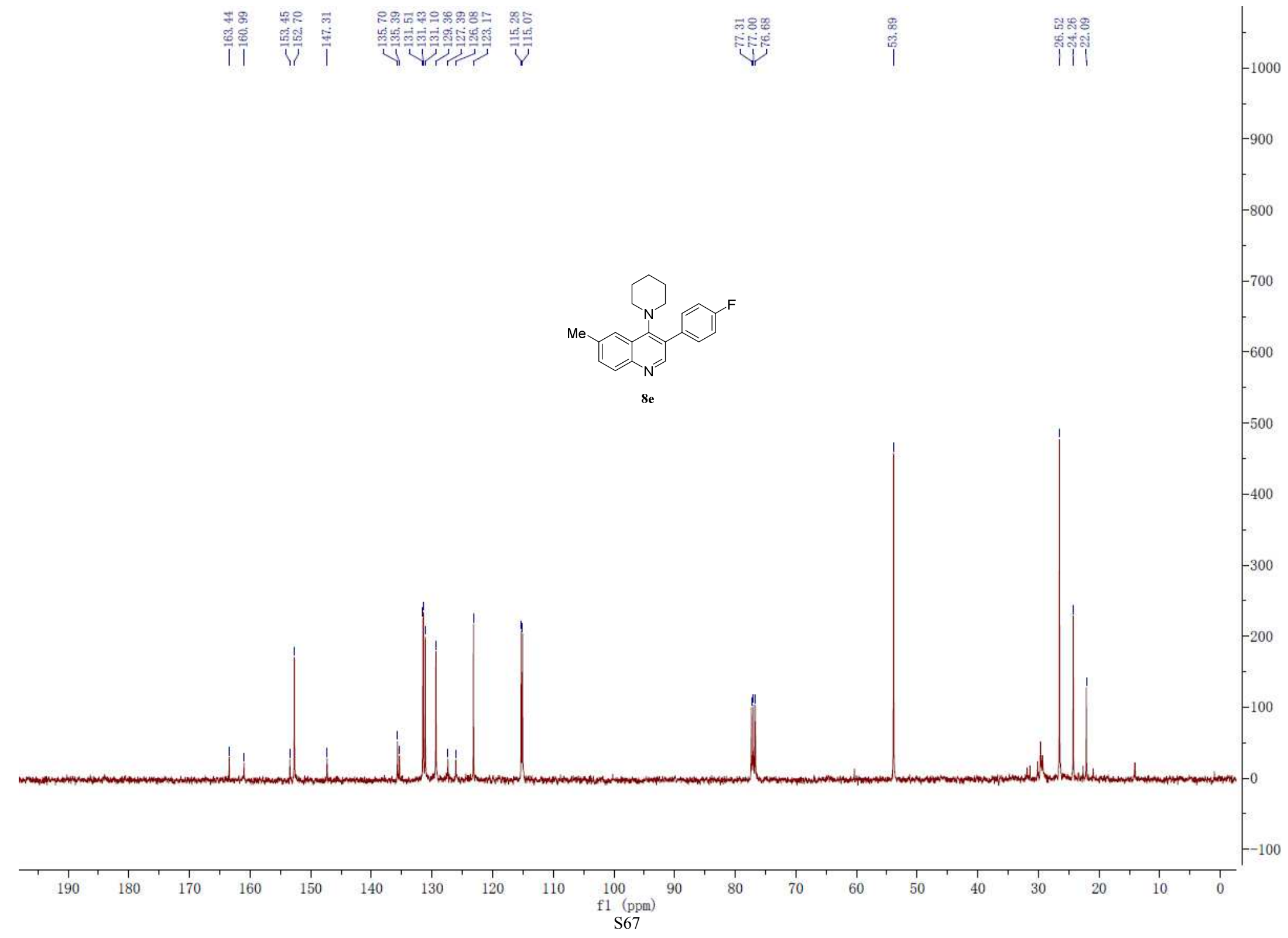




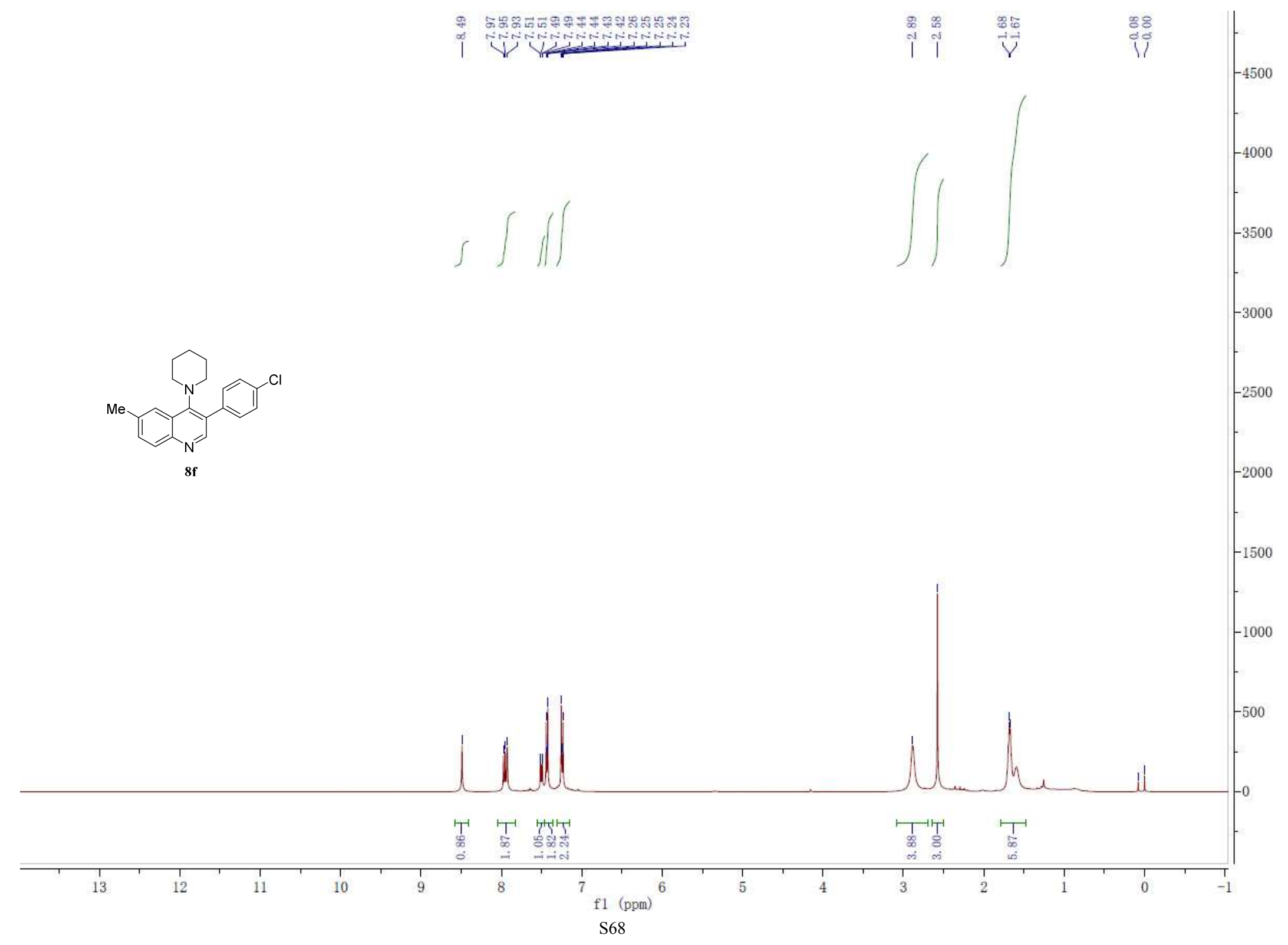




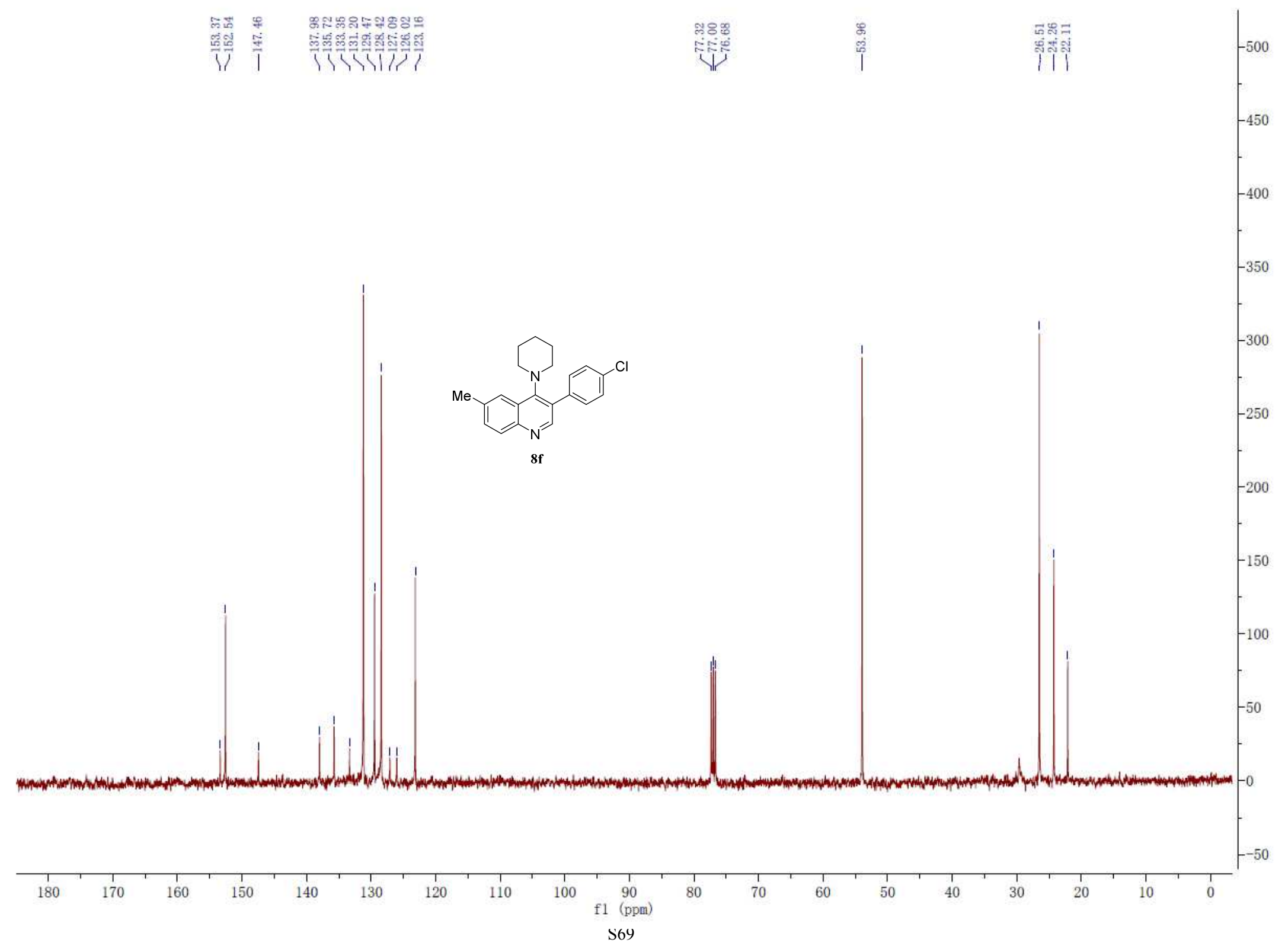




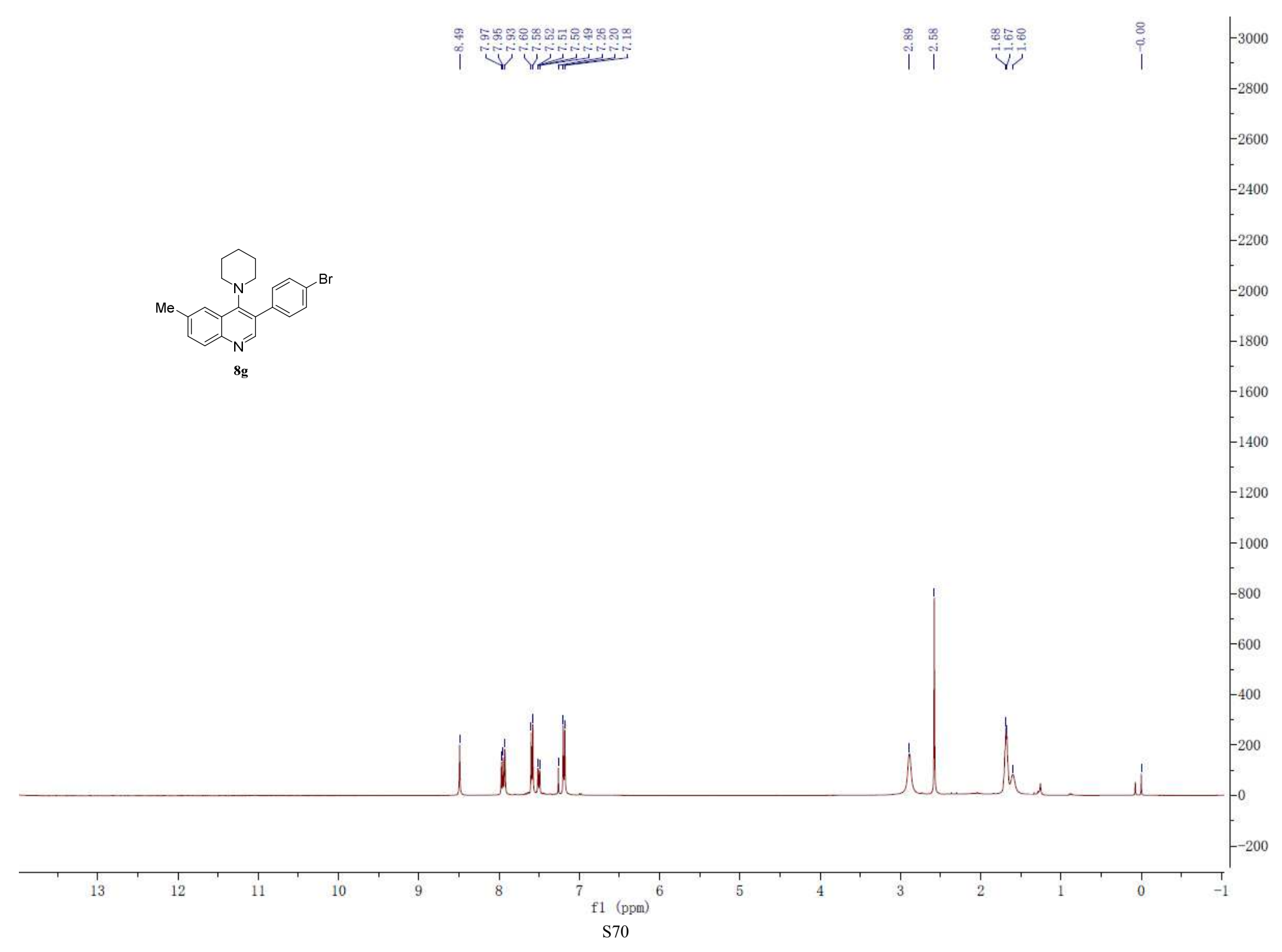




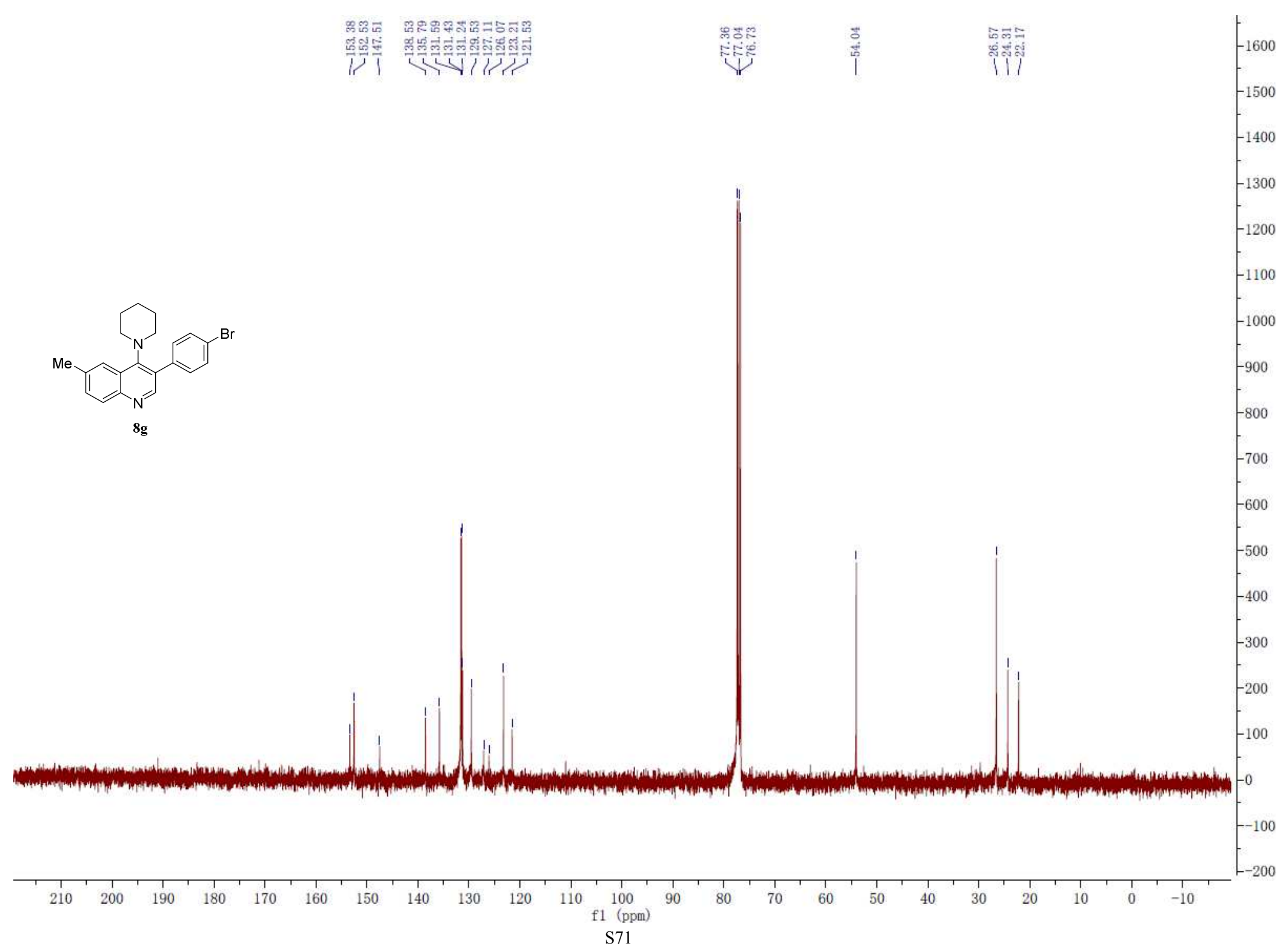




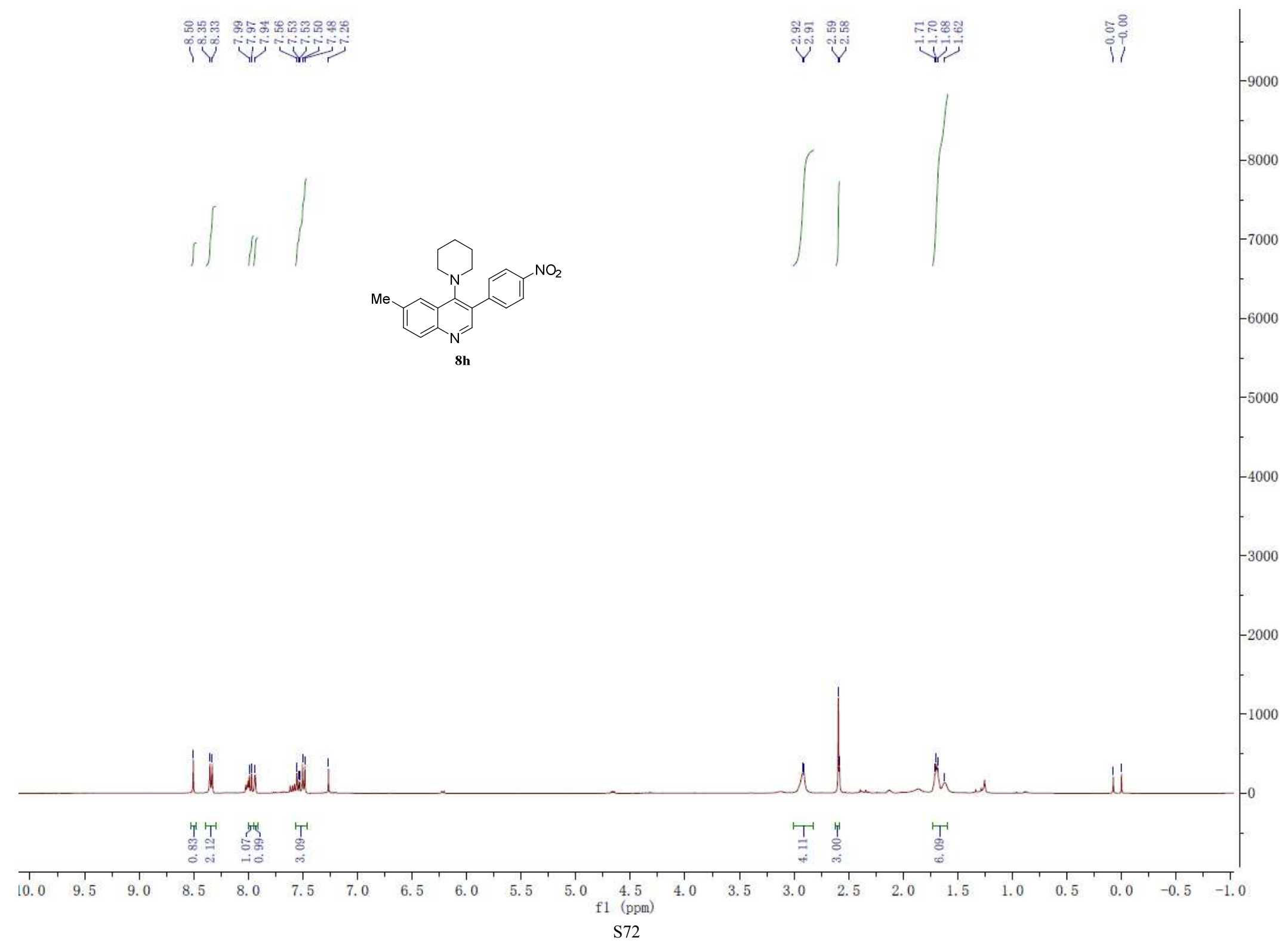




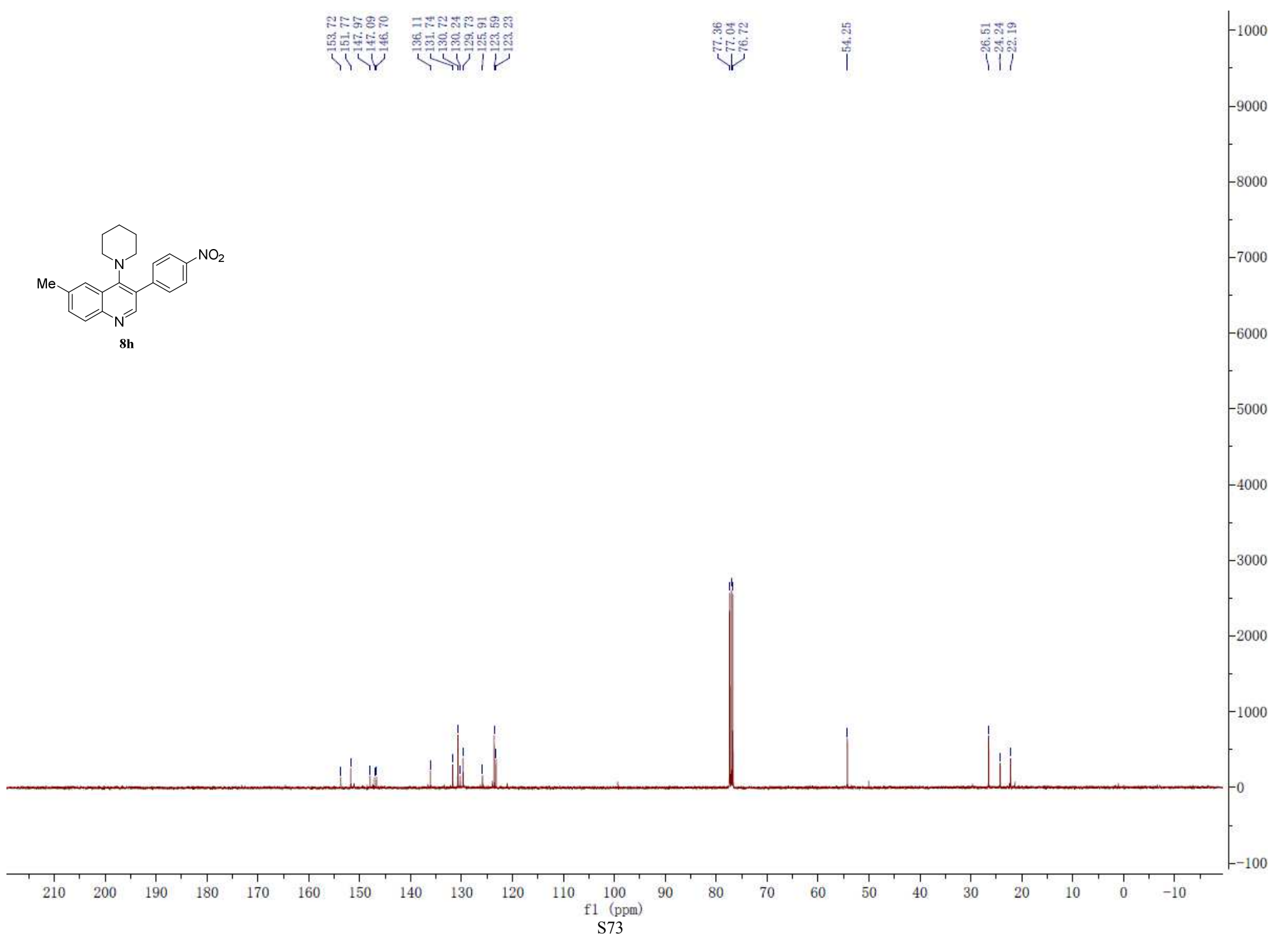




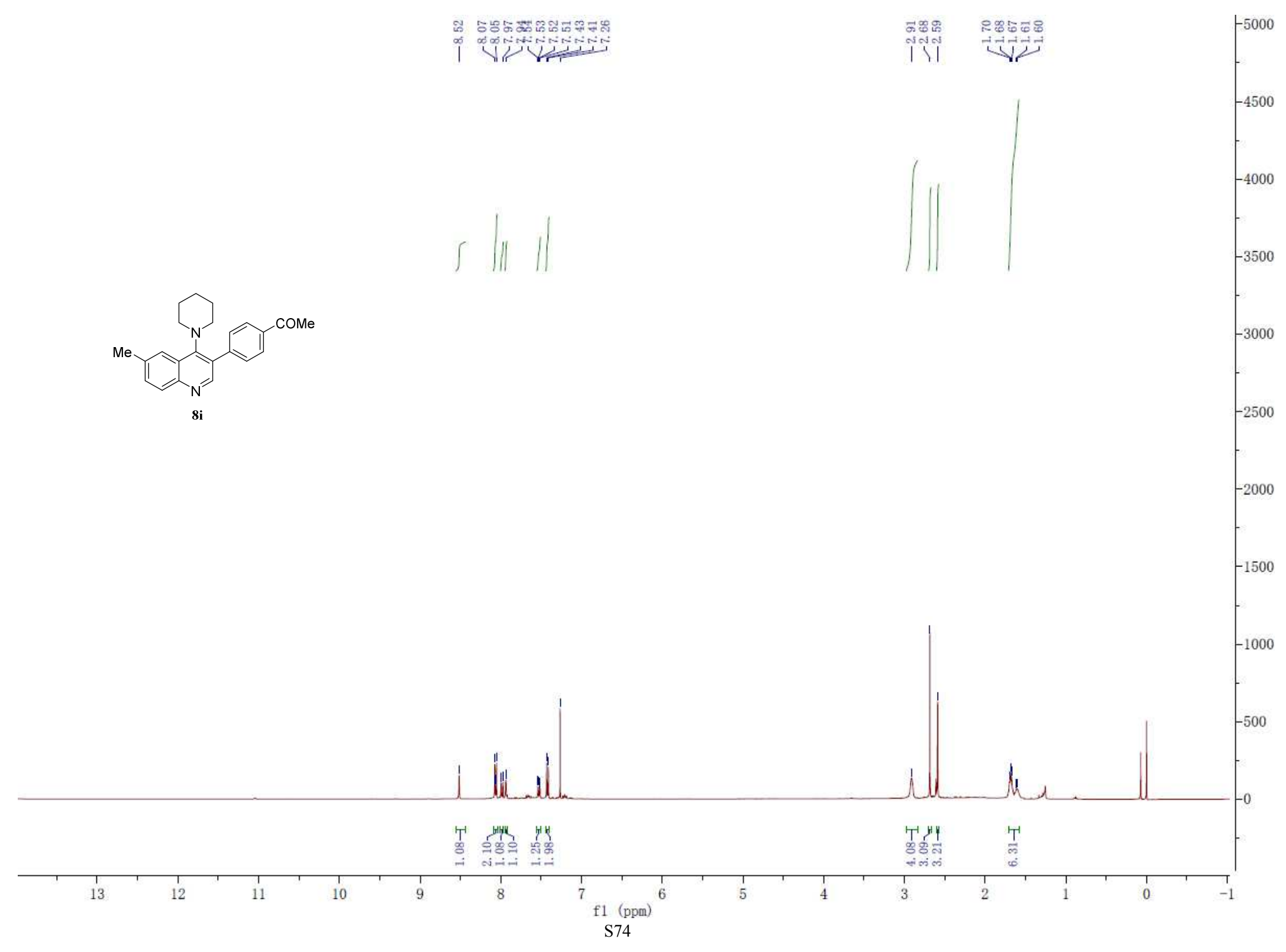




$$
+1
$$




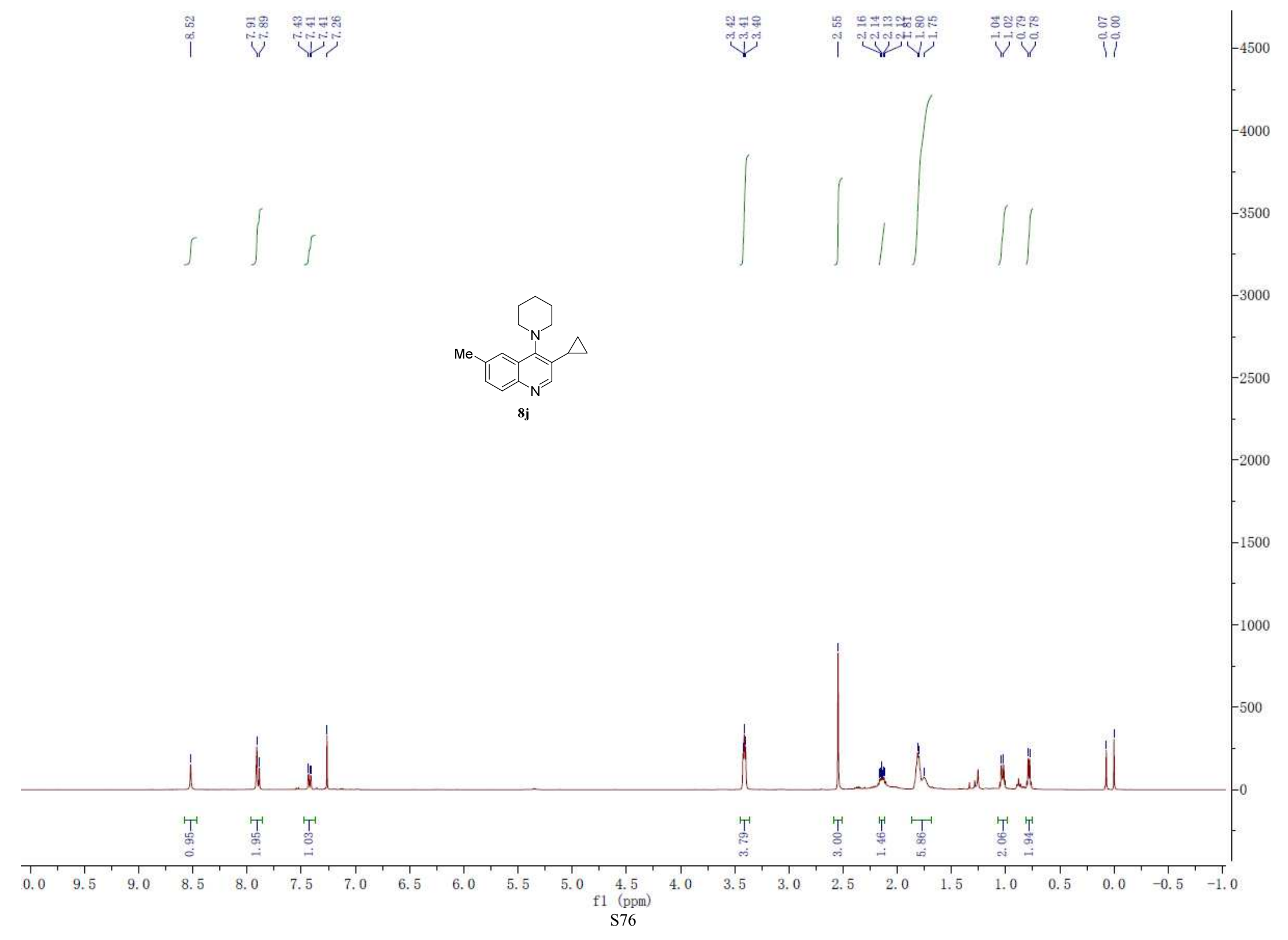




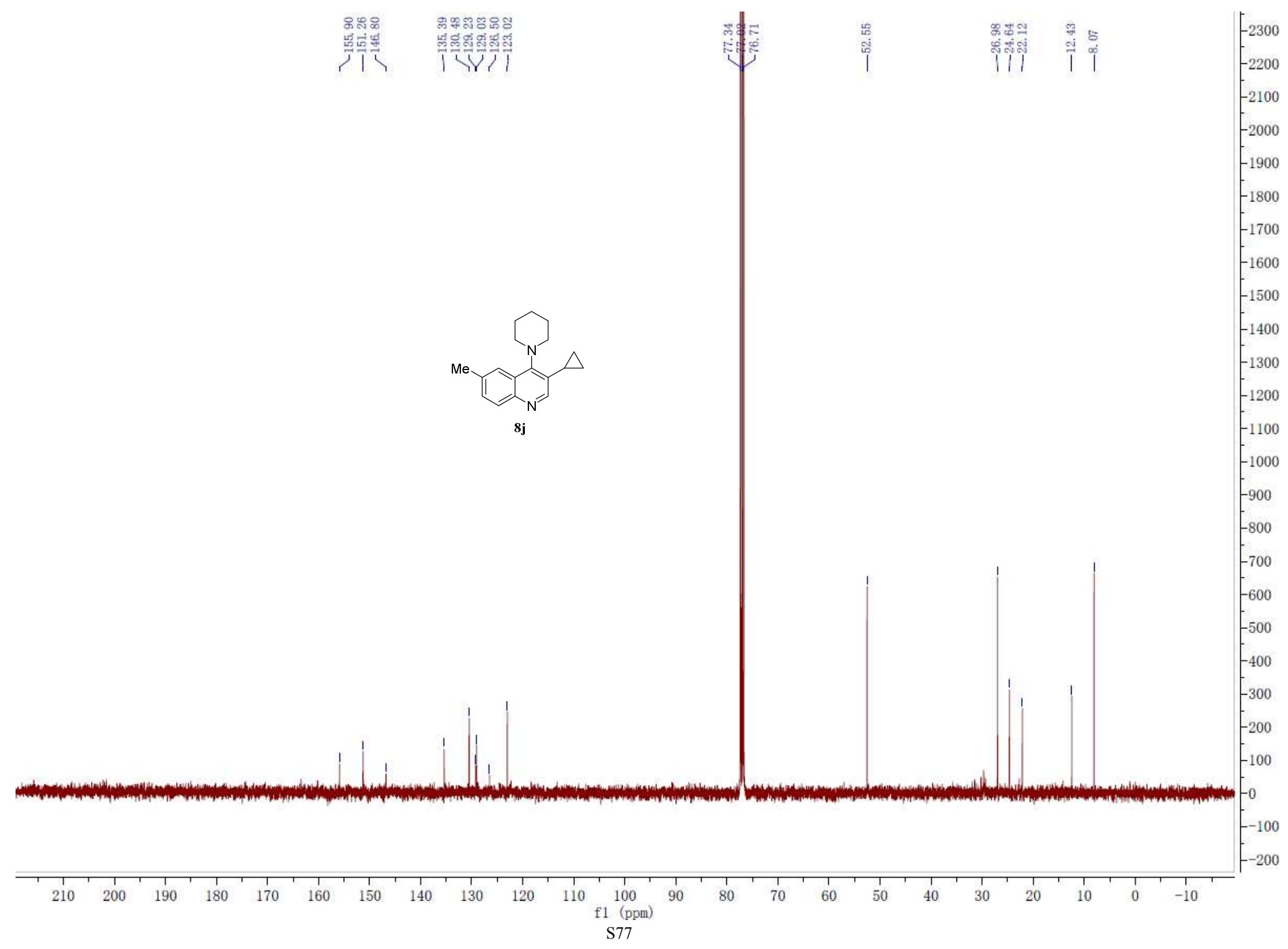

